\title{
Applications of ion mobility mass spectrometry for high throughput, high resolution glycan analysis
}

DOI:

10.1016/j.bbagen.2016.02.003

\section{Document Version}

Accepted author manuscript

Link to publication record in Manchester Research Explorer

\section{Citation for published version (APA):}

Gray, C., Thomas, B., Upton, A., Migas, L., Eyers, C. E., Barran, P., \& Flitsch, S. (2016). Applications of ion mobility mass spectrometry for high throughput, high resolution glycan analysis. Biochimica et Biophysica Acta (BBA)-General Subjects. https://doi.org/10.1016/j.bbagen.2016.02.003

\section{Published in:}

Biochimica et Biophysica Acta (BBA)-General Subjects

\section{Citing this paper}

Please note that where the full-text provided on Manchester Research Explorer is the Author Accepted Manuscript or Proof version this may differ from the final Published version. If citing, it is advised that you check and use the publisher's definitive version.

\section{General rights}

Copyright and moral rights for the publications made accessible in the Research Explorer are retained by the authors and/or other copyright owners and it is a condition of accessing publications that users recognise and abide by the legal requirements associated with these rights.

\section{Takedown policy}

If you believe that this document breaches copyright please refer to the University of Manchester's Takedown Procedures [http://man.ac.uk/04Y6Bo] or contact uml.scholarlycommunications@manchester.ac.uk providing relevant details, so we can investigate your claim.

\section{OPEN ACCESS}




\title{
Applications of ion mobility mass spectrometry for high throughput, high resolution glycan analysis
}

\author{
C.J. Gray, ${ }^{1 *}$ B. Thomas, ${ }^{1 *}$ R. Upton, ${ }^{1 *}$ L.G. Migas, ${ }^{1}$ C.E. Eyers, ${ }^{2}$ P.E. Barran ${ }^{1,3}$ and S.L. Flitsch ${ }^{1}$
}

${ }^{1}$ School of Chemistry \& Manchester Institute of Biotechnology, The University of Manchester, 131 Princess Street, Manchester, M1 7DN, United Kingdom

${ }^{2}$ Department of Biochemistry, Institute of Integrative Biology, University of Liverpool, Crown Street, Liverpool, L69 7ZB, United Kingdom

${ }^{3}$ Michael Barber Centre for Collaborative Mass Spectrometry, Manchester Institute of Biotechnology, The University of Manchester, 131 Princess Street, Manchester, M1 7DN, United Kingdom

*Denotes authors corresponded equally

\begin{abstract}
Background

Diverse varieties of often heterogenous glycans are ubiquitous in Nature. They play critical roles in recognition events, act as energy stores and provide structural stability at both molecular and cellular levels. Technologies capable of fully elucidating the structures of glycans are far behind the other 'omic' fields. Liquid chromatography (LC) and mass spectrometry (MS) are currently the most useful techniques for high-throughput analysis of glycans. However, these techniques do not provide full unambiguous structural information and instead the gap in full sequence assignment is frequently filled by a priori knowledge of the biosynthetic pathways and the assumption that these pathways are highly conserved.
\end{abstract}

\section{Scope of the review}

This comprehensive review details the rise of the emerging analytical technique ion mobility spectrometry (IMS) (coupled to MS) to facilitate the determination of three-dimensional shape: the separation and characterization of isobaric glycans, glyco(peptides/proteins), glycolipids, glycosaminoglycans and other polysaccharides; localization of sites of glycosylation; or interpretation of the conformational change to proteins upon glycan binding.

\section{Major conclusions}

IMS is a highly promising new analytical route, able to provide rapid isomeric separation (ms timescale) of either precursor or product ions facilitating MS characterization. This additional separation also enables the deconvolution of carbohydrate MS(/MS) information from contaminating ions, improving sensitivity and reducing chemical noise. Derivation of collision cross sections (CCS) from IM-MS(/MS) data and subsequent calculations validate putative structures of carbohydrates from $a b$ initio derived candidates. IM-MS has demonstrated that amounts of specific glycan isomers 
vary between disease states, which would be challenging to detect using standard analytical approaches.

\section{General significance}

IM-MS is a promising technique that fills an important gap within the Glycomics toolbox, namely identifying and differentiating the three-dimensional structure of chemically similar carbohydrates and glycoconjugates.

\section{Introduction}

Glycans and their conjugates (proteins, lipids, various anabolites) are found attached to the surface of cells, within cells and in the extracellular matrix. They are integral to an extraordinarily diverse range of biological processes including regulation of cell-cell recognition[1], cell adhesion[23] (including host-pathogen interactions[4-6]), immune response,[7] fertilization,[4, 8-10] trafficking, and intra- and extracellular signalling events. Even subtle changes in carbohydrate structure can result in vastly different interactions, make them susceptible to enzymatic hydrolysis or alter glycoconjugate tertiary structures and thus affect their observed biological response.[2, 11-18] Interestingly both the identity and levels (or presence) of specific glycan structures within individuals depends on numerous factors including gender,[8, 19] blood group,[20] age,[21-23] disease state[12, 17, 24-28] and diet.[29-30] It remains unclear a priori how changes in glycan structure will affect the resultant biological function.[31] Given their ubiquity in nature, it is unsurprising that aberrant glycan structures have been identified as biomarkers for several disease states including various cancers, [11, 14, 26, 32-36] hereditary disorders,[17, 37] acute pancreatitis,[24] immune and cardiovascular deficiencies,[12, 31, 38] Alzheimer's disease[39-40] and muscular dystrophy[2].

The incredible amount of three dimensional stereochemical information contained within glycans is biologically very important, as it is required to accommodate for their diverse functionalities even from a relatively small pool of monosaccharide building blocks. This complex 'chemical information' arises from: (1) different monosaccharide building blocks; (2) the potential to link these monomers at a number of different positions in two different stereochemistries, namely $\alpha$ and $\beta$-, forming both linear and branched structures; (3) the ability for identical monosaccharides to have been incorporated with different ring connectivities, i.e. pyranose and furanose structures (4) further decoration with chemically diverse functional groups (e.g. phosphate, sulphate, methyl groups) (Figure 1). To understand and exploit glycan-driven functions, it is necessary to be able to unambiguously elucidate carbohydrate structures, in the absence of a priori information to be able to define structure-function relationships. To develop and exploit differences in glycan structures as potential biomarkers of disease, it will be necessary to define these structures in a high throughput manner to allow characterization on a person-to-person basis, permitting the development of novel 
therapeutics or diagnostics.[41-43] The demand for three-dimensional structure determination has resulted in fewer advances in comprehensive structural elucidation of glycans compared with proteins and nucleic acids, especially since glycan structures are not directly encoded by genetic information. Moreover, microheterogeneity, the low amounts available for analysis, and the chemical similarity between monosaccharide building blocks, which are often simple epimers of one another, hinder their full structural elucidation. 

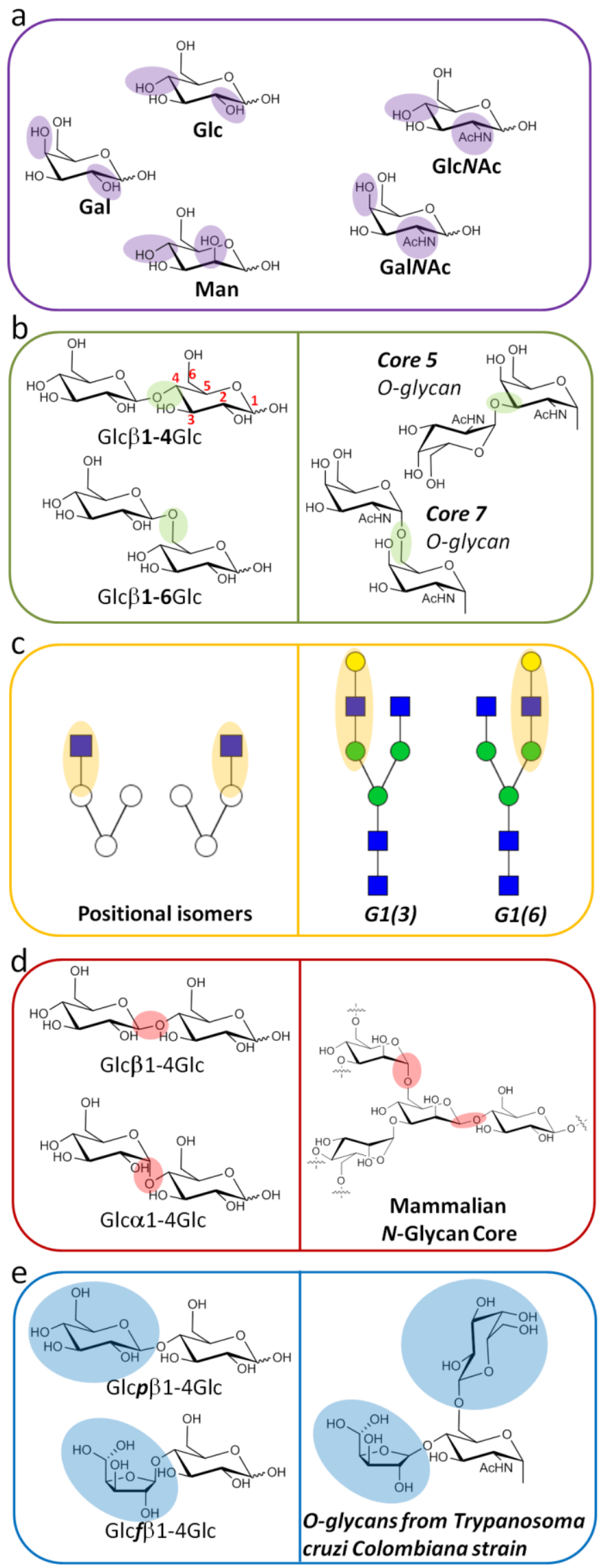
Figure 1 - Scheme depicting the various types of isomerism routinely observed in nature. The left side of the panel highlights the types of isomerism observed and the right side displays naturally occurring examples. Different stereochemistries associated with the monosaccharide building blocks (a) Regioisomers of glycosidic bond formation between two monosaccharide building blocks, which includes the potential formation of branched structures; (b) Positional isomers resulting from addition of a monosaccharide unit to different branching arms; (c) Anomeric glycosidic bond isomers $\alpha$ - and $\beta$-; (d) Finally, ring size isomers pyranose, $p$, and furanose, $f,(e)$.

The current 'gold standard' for glycan analysis typically involves liquid chromatography (LC) separation,[11-12, 31, 44-45] which can also be coupled with the speed and sensitivity of tandem mass spectrometry $\left(\mathrm{MS}^{2}\right.$ ) (Figure 2).[46-48] Glycans are then characterized based on either their retention time against a standard and/or by their $\mathrm{MS}^{2}$ spectrum.[45, 49-57] However, LC separation can require multiple different columns to gain significant separation of chemically similar glycan species and as a result it is often low throughput. Also, without the benefit of (synthetic) reference standards, LC provides no structural de novo information. MS alone is limited to characterising the monosaccharide class (i.e. hexose, N-acetylhexosamine, deoxy hexose etc.) and is insufficient to directly identify these monosaccharide units from each other without the use of an orthogonal sequencing approach. Given the intrinsic ability of carbohydrates to be isomeric, these strategies only often work for systems whose biosynthetic pathways are well understood (e.g. N-glycans) to facilitate in their identification. The inability of this analysis strategy to separate and characterize these iso(mer/bar)ic species from complex biological mixtures can hinder the development of specific glycomic approaches for assessing disease state.[27]

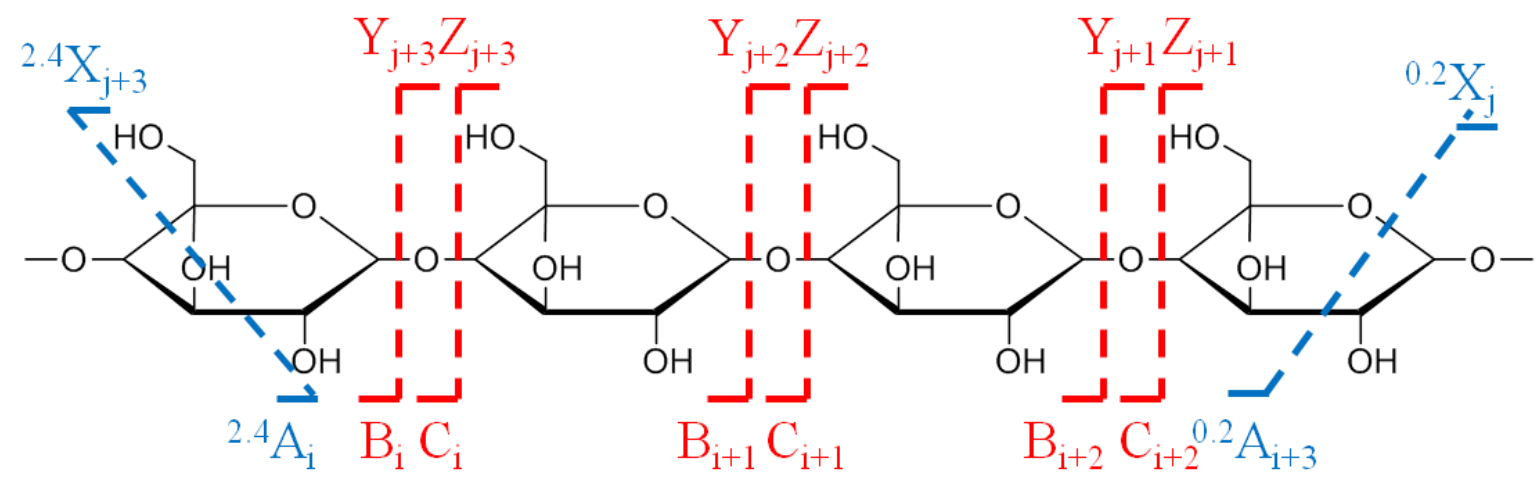

Figure 2 - Scheme depicting Domon-Costello nomenclature for carbohydrate product ions formed as a result of tandem mass spectrometry. Red and blue ions correspond to those formed as a result of fragmenting the glycosidic bond and the monosaccharide ring respectively.[58]

Recently there has been a surge in the application of Ion Mobility-Mass Spectrometry (IMMS) to enhance structural characterization and separation of glycans and glycoconjugates. Ion mobility spectrometry (IMS) is an analytical technique that measures the mobility, $K$, of gas-phase ions under the influence of an electric field in the presence of a buffer gas; the mobility is based on the size, shape and charge of the ions,[59-60] analogous to electrophoresis in the condensed phase, although the timescale is much shorter (high- $\mu$ s to $\mathrm{ms}$ ). Ions can be generated by a range of techniques, although for most IMS applications, ions are generated by ESI. This technique has been used for a variety of types of analyses, and has major applications in security and defence for example 
in airports where it is routinely used to screen against explosives, chemical-warfare agents and drugs. The recent surge in use of ion mobility to analyse glycans is primarily as a result of the commercialization of hybrid IM-MS instrumentation (Synapt HDMS, 2006) and its potential to overcome challenges associated with analysis of glycans; namely separation of often isomeric carbohydrates and structural characterization of carbohydrates in conjugation with molecular dynamics (MD) simulations.

This review assesses the applicability of IM-MS towards biologically relevant glycans and the wealth of additional information that can be obtained from this high-throughput strategy. This will undoubtedly improve our understanding of glycan functions and thus could provide promising tools for further medicinal applications. Initially, the various IMS strategies routinely coupled to MS will be briefly discussed to help the reader appreciate the limitations and advantages of each of the techniques; however for a more thorough background into IMS the reader is encouraged to read excellent previous reviews.[60-62] Similarly, computational aspects (such as MD simulations) that facilitate elucidation of gas-phase structures are briefly discussed, however, they are more extensively detailed elsewhere.[63-64]

\section{Ion mobility spectrometry}

A number of unique ion mobility analysers have been coupled to MS, each with their own benefits and limitations (Figure 3). Each of these analysers are discussed in further detail.
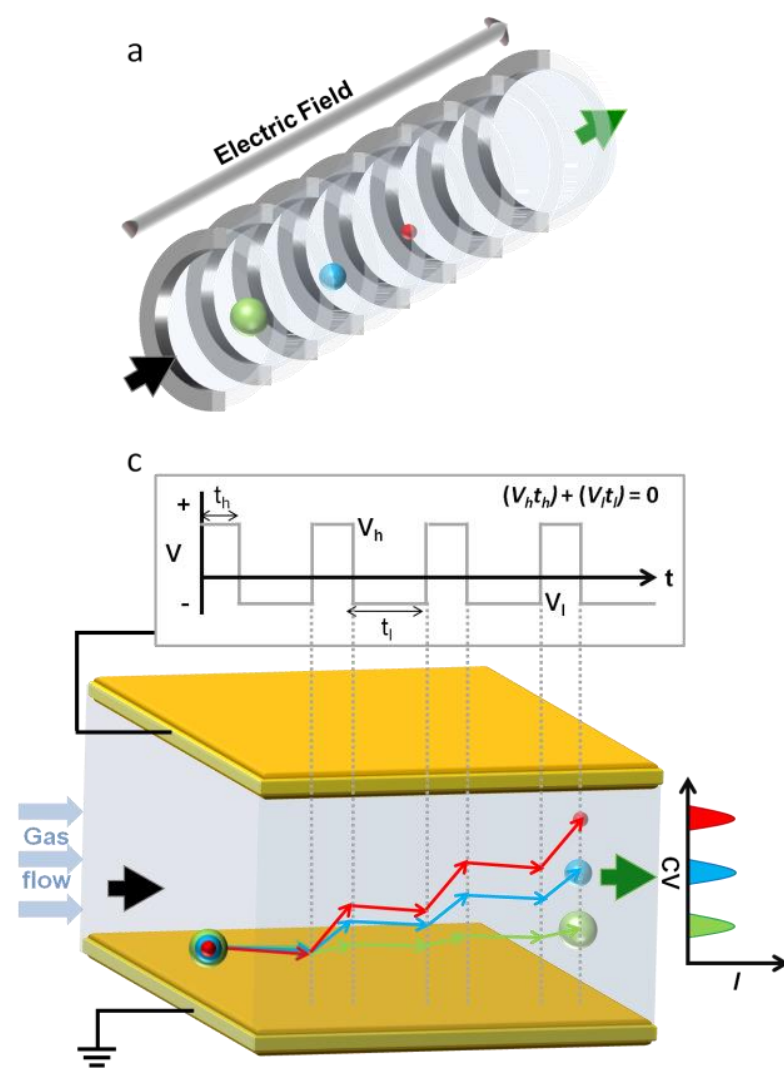

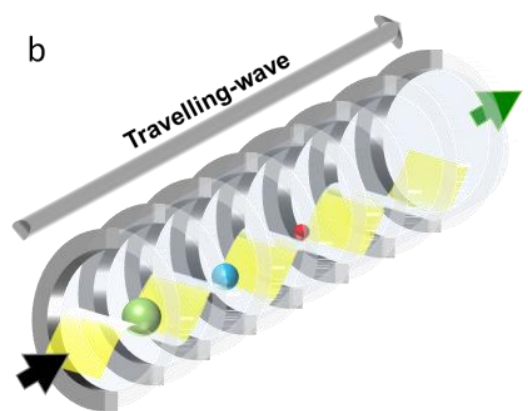

d

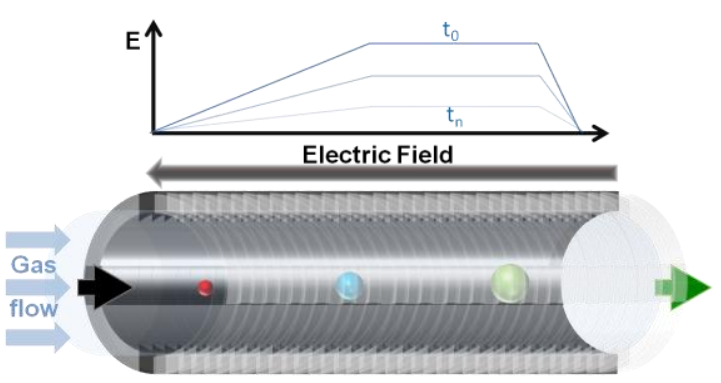


Figure 3 - Four different mobility cells for drift tube (a), travelling-wave (b), (high) field asymmetric (c) and trapped (d) ion mobility spectrometry. Ions of increasing collision cross section $(\Omega)$-to-charge ratio are depicted as red, blue and green spheres. Solid black and green arrows correspond to where the ions enter and exit respectively.

\subsection{Drift tube (DT)IMS}

DTIMS is conceptually the simplest form of IMS. Ions are introduced (gated) into a cell consisting of a series of stacked electrodes filled with a static drift/buffer gas. A weak, uniform electric field $(\sim 5-15 \mathrm{~V} / \mathrm{cm})$ is applied across the cell causing the ion packet to move through the cell. Ions with larger rotationally averaged collision cross section (CCS) undergo more collision/interaction events with the drift/buffer gas and as a result take longer to traverse the cell. This CCS represents the effective area of the gas-phase ion, under the precise set of experimental conditions that can interact with the drift gas, averaged over all orientations and thus can be related to structural or conformational features. Mobility separated ions are then separated by their $\mathrm{m} / \mathrm{z}$ using a mass analyser that tends to be, but not exclusively, a time-of-flight device before being detected. The mobility or reduced mobility, $K_{0}$, for a standard set of conditions can then be calculated for the ions using equations 1 and 2 :

$$
\begin{gathered}
K=\frac{L}{t_{d} E} \\
K_{0}=K \frac{P}{760} \frac{273.2}{T}=\frac{L}{t_{d} E} \frac{P}{760} \frac{273.2}{T}
\end{gathered}
$$

Where $L$ is the length of the drift cell, $t_{d}$ is the drift time, $E$ is the electric field, $P$ is the pressure within the cell and $T$ is the temperature of the buffer gas within the cell. $K_{0}$ can then be equated to the rotationally averaged CCS under a specific set of instrument conditions, $\Omega$, according to the MasonSchamp equation (3):[65-66]

$$
\Omega=\frac{1}{K_{0}} \frac{(18 \pi)^{1 / 2}}{16} \frac{z e}{\left(k_{b} T\right)^{1 / 2}}\left[\frac{1}{m_{I}}+\frac{1}{m_{B}}\right]^{1 / 2} \frac{1}{N}
$$

Where $k_{b}$ is the Boltzmann constant, $m_{I}$ is the mass of the ion, $m_{B}$ is the mass of the buffer gas, $z$ is the charge state, $e$ is the elementary charge, and $N$ is the number density of the drift gas; this relationship only holds true for low $E / N(<2$ Td).[67-68] [69-70] At high $E / N$ the electric field causes ion heating and therefore a non-linear relationship between $1 / K_{0}$ and $\Omega$. 


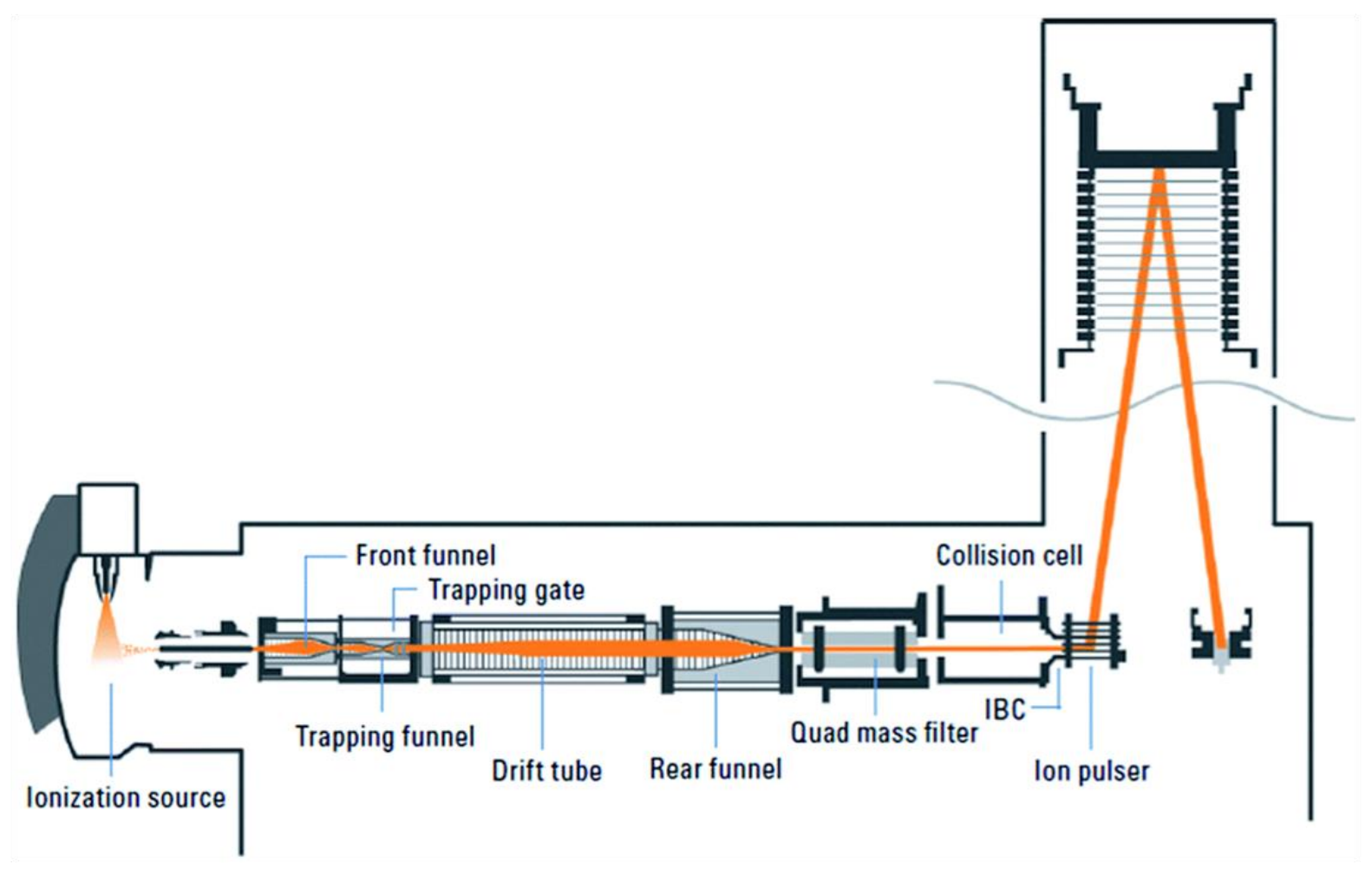

Figure 4 - Schematic of the commercialially available Agilent DTIMS instrument.

The resolving power of IMS instrumentation is highly dependent on the choice of buffer gas. Classically for gas-phase structural (and separation) analysis, the buffer gas is an inert gas such as helium or nitrogen; these two gases have been extensively studied and permit determination of the CCS of ions of interest. In the past, other gases have been shown to offer superior separation capabilities, particularly gases that are polarizable (i.e. $\mathrm{CO}_{2}, \mathrm{SF}_{6}$ and $\mathrm{N}_{2} \mathrm{O}$ ). These gases allow for the improved separation of isomeric species, but this separation is highly dependent on the physical properties of the analyte.[66] The resolving power of modern IMS instruments tends to be in the order of 50-100 $(\Omega / \Delta \Omega)$ and the increase in resolution tends to come at the expense of sensitivity. One major disadvantage of DTIMS is its low duty cycle. The DTIMS instrument has only been used by a handful of specialized research groups due to the lack of commercially available instrumentation, however, with the recent release of a commercial instrument (Agilent 6560 Ion Mobility QTOF;Figure 4), adoption of DTIMS and its application is expected to rise.[71]

\subsection{Travelling wave (TW)IMS}

TWIMS was first commercialized by the release of the Synapt HDMS (2006, Waters;Figure 5).[72] Since then several new improved iterations of the Synapt HDMS series and other instrumentation possessing TWIMS (i.e. Waters, Vion IMS QToF) have been released.[73] Ions are guided through the mobility cell using a series of ring electrodes (stacked ring ion guide), in the presence of a static buffer gas (commonly $\mathrm{N}_{2}$ ) akin to DTIMS. Travelling waves are generated by 
raising the voltage on selected, periodically spaced electrodes and then moving the potential along the ring electrodes. Ions with smaller CCS undergo fewer collisions with the drift gas and 'surf' the waves, whereas larger ions undergo more collisions and are more likely to tumble over the back of the waves. Simultaneously a RF potential is applied to the electrodes creating a well at the centre of the cell, radially confining ions and preventing them from diffusing to the electrodes where they would annihilate. Mobility separation can be tuned by altering the wave height, velocity or the pressure of the mobility cell. Like DTIMS, TWIMS operates in the low E/N regime meaning CCS can be derived. However, ion trajectories through the mobility cell have not been fully elucidated and as a result the CCS cannot be directly measured from the drift time of the analyte. As a result, CCS derivation by TWIMS is achieved by calibration against a series of molecules whose CCS are known and ideally are of similar 'class' to the analyte being studied.[74-75] When multiple charge states are present in either the calibrant or analyte, their CCS is often reported as a mass and charge state normalized value $\left(\Omega^{\prime}\right)$ calculated using the following equation (4):

$$
\Omega^{\prime}=\frac{\Omega(\mu)^{1 / 2}}{z}
$$

where $\mu$ is the reduced mass. However, using $\Omega^{\prime}$ values for calibration have been reported to introduce significant errors.[74] Unfortunately for structural applications, TWIMS data is commonly recorded in nitrogen and DTIMS in helium. TWIMS cannot operate with (pure) helium as a drift gas since the cell operates at a potential and pressure beyond the electrical breakdown limit of helium. As a result ${ }^{\mathrm{TW}} \mathrm{CCS}_{\mathrm{N} 2}$ is often converted to a representative ${ }^{\mathrm{TW}} \mathrm{CCS}_{\mathrm{He}}$ value for calibration purposes, although this is also reported to introduce error.[75-76] The T-wave cell is also reported to produce significant ion heating, raising questions on the ability of the instrument to yield structural data representative of the solution phase ("native"). However, recent advances of the instrument design have been demonstrated to significantly reduce ion heating, with evidence that solution-phase protein, peptide and glycan conformers can be retained in the gas-phase.[77-84] Despite these concerns, the ion heating is insufficient to break the covalent bonds, therefore TWIMS is a suitable technique for structural analysis of carbohydrates. 


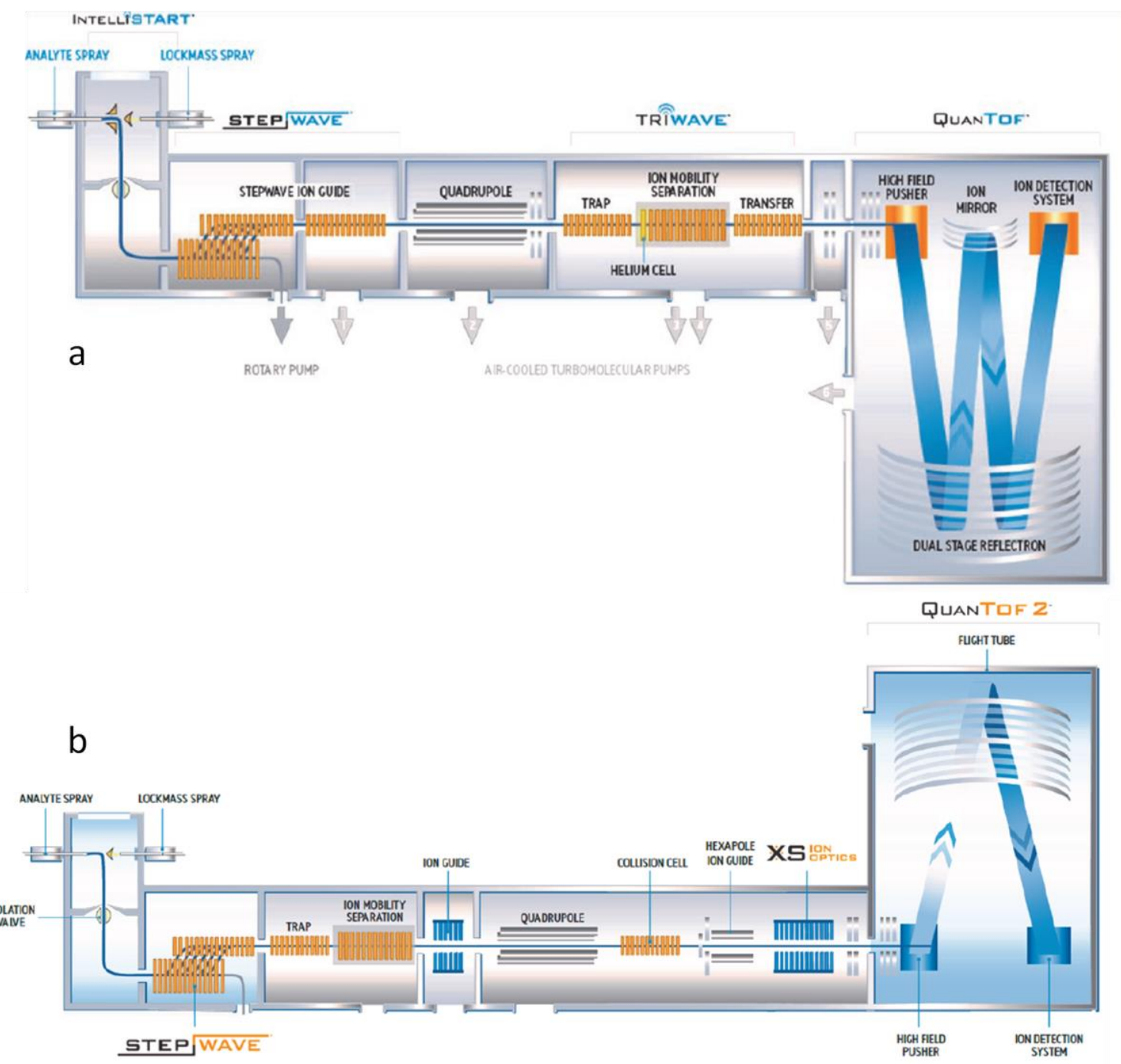

Figure 5 - Schematic of the Synapt G2-Si (a) and Vion (b) TWIMS mass spectrometers commercialized by Waters.

The resolving power of TWIMS ( 40) is worse when compared to typical DTIMS analysers, however its duty cycle and sensitivity are much greater. Another convenient facet of the Synapt instrument series is the presence of collision cells before and after the mobility cell (denoted TRAP and TRANSFER, respectively), enabling mobility separation of either precursor or product ions.

\section{3 (High-)Field asymmetric (FA)IMS}

FAIMS or differential ion mobility spectrometry, unlike DTIMS and TWIMS, operates at high field (high $E / N,>30 \mathrm{Td}[85]$ ) and therefore CCS cannot be derived from the mobility data. As a result, FAIMS is always used as a gas-phase separation technique at the front-end of the mass spectrometer. FAIMS achieves ion separation based on the differential mobility operating in low field and high field conditions. Ions are carried by a drift gas between two parallel plates; one plate is held at ground whilst an asymmetric waveform is applied to the other. This waveform consists of a high potential (high-field) for a short time period followed by application of a low potential of opposing 
polarity for a longer period of time. These time periods and potentials are designed so that $\left(V_{h} t_{h}\right)+$ $\left(V_{l} t_{l}\right)=0$ where $V_{h}$ and $V_{l}$ are the voltages applied during the high-field and low-field period respectively, and $t_{h}$ and $t_{l}$ are the time periods for the high-field and low-field portions of the waveform respectively. This ensures that mobility separation is resulting solely from the difference in mobility at high- and low-fields.[86] A compensation voltage (CV) is applied allowing transmission of ions that have been displaced through their differential mobility by a specific amount. As a result, FAIMS can operate as an ion mobility filter akin to quadrupoles acting as $m / z$ filters. In this mode the sensitivity is much greater (higher duty cycle) as it can separate ion beams rather than ion packets. Conversely the compensation voltage can be scanned to allow detection of all ions, although this reduces the sensitivity. The other major advantage of this technique is that it currently has one of the highest reported resolving powers that has been reported for an IMS device (>500),[87] with resolving powers routinely reported to be $\sim 100$. Like TWIMS, the drift gas employed in FAIMS separation is typically nitrogen to prevent electrical breakdown,[88] however helium or hydrogen can be doped into nitrogen, which was reported to improve the resolving power of the instrument.[87] Alternatively, chips with narrower channels could allow the use of helium.[89]

\subsection{Trapped IMS (TIMS)}

The final IMS technique that shall be discussed is the recently developed trapped IMS (TIMS). Ions are pulsed (with a deflection plate) into an ion funnel, where they are radially focused before entering the IMS region (Figure 6).[90-91] Ions enter the TIMS region, which consists of a series of airtight plates segmented into quadrants, with a flow of drift gas. An axial electric field opposing the drift gas flow is applied trapping ions at different regions within the TIMS cell depending on the ions $\Omega / z$. Simultaneously, an RF potential is applied to the quadrants, creating a quadrupole field perpendicular to the electric field. This quadrupole field restricts ions to the centre of the cell. The axial electric field is then steadily reduced eluting species from high to low $\Omega / z[90]$ meaning TIMS can be used as an IMS filter, like FAIMS. Since TIMS operates at the low-field limit, CCS values can be extracted from the data (via calibration to known CCS standards), similar to TWIMS.

Considering its only recent development and commercialization there are still very few publications in the area, especially those related to carbohydrate separation. However, TIMS has been reported to offer high resolving powers (>250), surpassing that of the other IMS devices (DT- and TW-IMS).[92] 


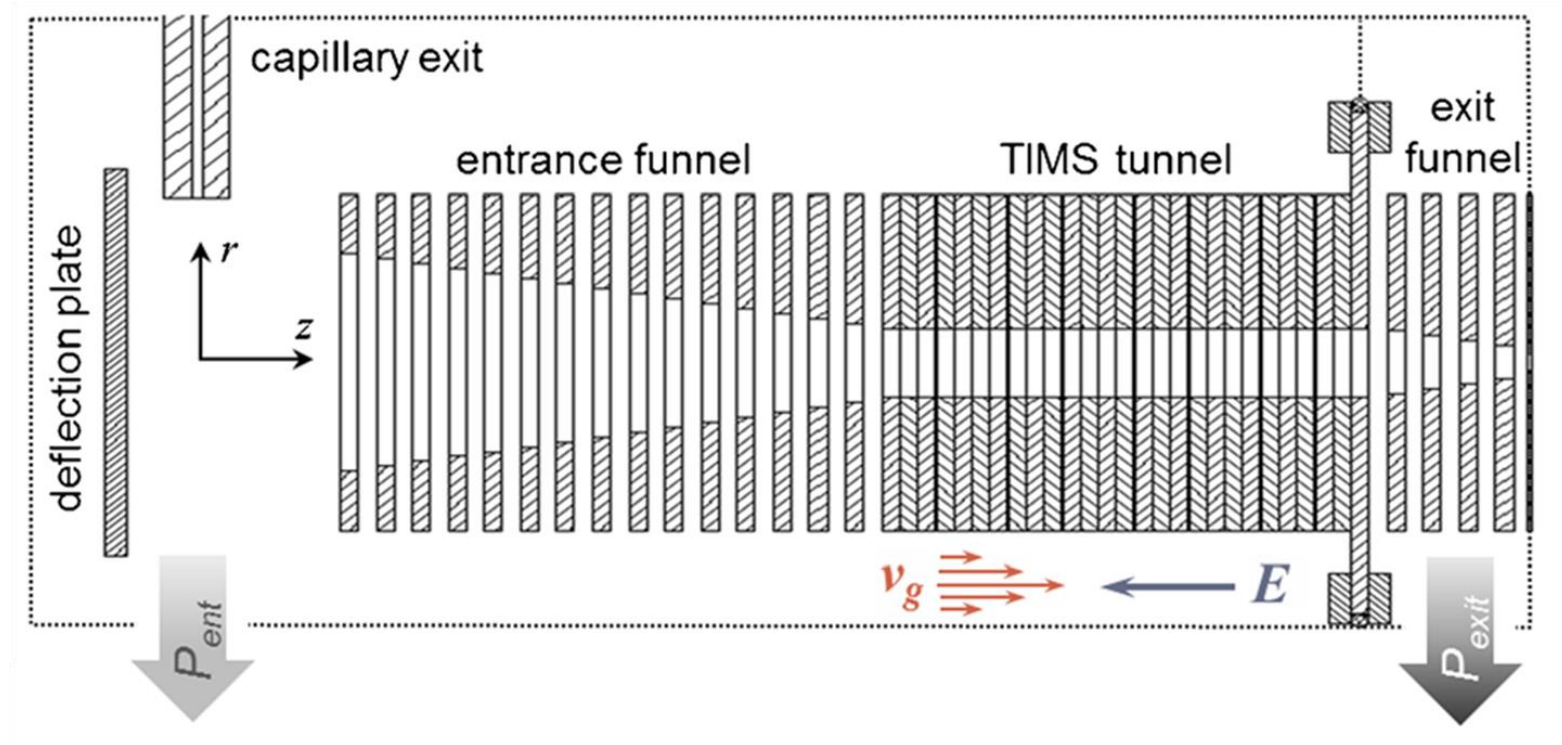

Figure 6 - Scheme of the TIMS cell devloped by Bruker that can be coupled onto the front-end of most instrumentation.

\section{Computational methodologies}

IM measurements alone do not directly reveal any structural insights beyond the relative size of measured ions and their conformational dynamics under the defined set of experimental conditions used. Instead, the CCS of computationally derived structures is compared to the experimental values to elucidate structural information. Theoretical CCS of an ensemble of structures that fit within the CCS distribution may indeed represent the gas-phase structure of the given analyte, assuming the energy of the structure is not unrealistically high. Normally, a set of structures is extracted from the conformational search, with particular focus on the more likely, low energy species. The information gathered from IM-MS ${ }^{\mathrm{n}}$ experiments combined with computational techniques such as ab initio, semiempirical or molecular dynamics simulations can offer powerful insights into the physical features of the molecules, including their connectivity, relative size and conformational dynamics.[93]

Despite significant progress being made in the field, generation of feasible models is not a trivial task, and goes beyond the scope of this review. For large macromolecular species, such as proteins, the initial modelled structures are normally taken from X-ray crystallographic databases or NMR atomic coordinates, as structure elucidation from primary sequence information alone is extremely difficult.

Modelling of smaller molecules, such as glycans, is achievable although computationally challenging even for mono- and di-saccharides. As expected, the main difficulty comes from the high diversity and flexibility of these species. 
The CCS values for the generated models are normally based on one of the following theoretical methods; Projection Approximation (PA),[94-95] Exact Hard Sphere Scattering (EHSS), ${ }^{[45]}$ Trajectory Method (TM)[96] or the Projection Superposition Approximation (PSA)[97-101] (Figure 7).[64] The choice of algorithm largely depends upon the physical properties and size of the analyte but also the computational resources available. The PA was the first theoretical method used to calculate CCS from atomic coordinates. Simplistically, the molecule is held in a fixed position and the atomic 'shadow' it casts onto a 2-dimensional plane is probed with a buffer gas; if the buffer gas was to collide with the 'shadow' the collision is counted as a hit and is measured. The molecule is randomly rotated around its axis, which is repeated multiple times to generate a rotationally averaged shadow or projection of the shape of the molecule, and is stopped once defined convergence criteria are met.[94-95]

The PA method is computationally least demanding, and offers the simplest and quickest approximation of the CCS, however, it does not account for the multiple scattering events that might occur during the collision and ignores all long-range interactions with the buffer gas. Slightly more sophisticated of the theoretical methods is the EHSS which treats molecules and the drift gas as rigid hard spheres, with pre-determined radii. Unlike PA, this method takes into account the multiple scattering events, although still does not take into account the interaction potentials of the system with the buffer gas. As a result EHSS remains computationally inexpensive, whilst offering better approximation.[102-104] The most commonly used method to study small molecules is the TM, which accounts for the long-range interactions during the collisions of the molecule with the buffer gas. The TM is typically the most reliable algorithm to theoretically derive CCS, however, it is also the most computationally demanding.[96] The TM has been parameterized and evaluated for helium and nitrogen, which are typically used in the IMS experiments; the parameters defined in the TM as implemented by Mesleh (for helium)[96] and Campuzano (for nitrogen)[105] are limited to several atomic types, meaning only certain classes of molecule can be accurately examined. The typical approach to overcome this limitation has been to define the parameters for unknown atoms yourself, or replace them with similarly sized atoms (compromising the accuracy of the calculated CCS). Finally the PSA, which was developed by Bleiholder et al., includes atomic potentials and a shape factor within the more simplistic and computationally efficient PA algorithm.[97-101] This method outperforms the EHSS and agrees relatively well with the TM, however is computationally 100-1000 times quicker making it an attractive alternative for larger systems such as glycoproteins or polysaccharides.[97] 

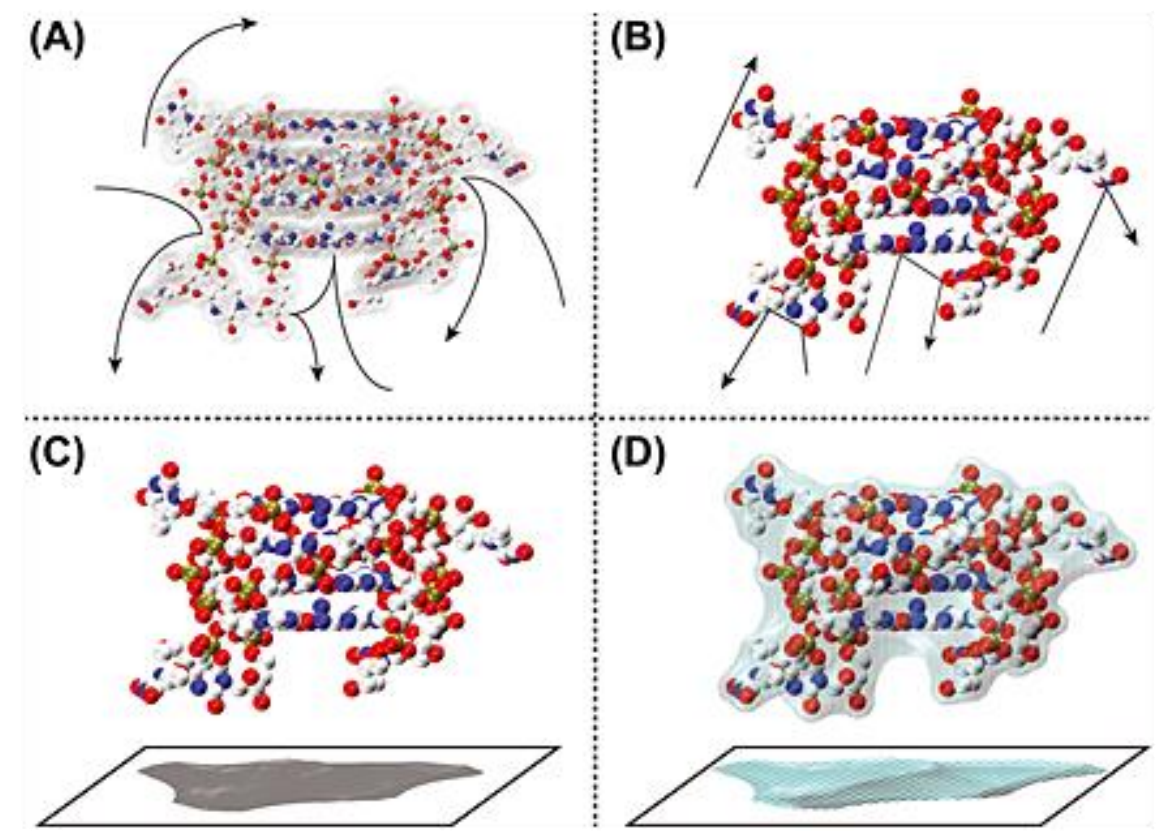

Figure 7 - Schematic of how the different models calculate CCS. The trajectory model, where the long range interactions between the drift gas and analyte ion are taken into account when determining the gas deflection trajectories and scattering angles (a). Exact hard sphere scattering model, where atoms within the analyte are considered as hard spheres and scattering angles between the drift gas and analyte are calculated (b). Projection approximation where the atoms are considered as hard spheres and the projected 'shadow' of all possible analyte orientations is calculated (c). Finally, the projection superimposition approximation which is similar to the PA, except atoms are considered as 'soft' spheres, accounting for the collective size effect by superimposition of all atomic contributions. The resulting value is scaled by a shape factor to account for molecular cavities (d). Figure taken from D'Atri et al.[64]

\section{Application of IMS to analysis of carbohydrates and their conjugates}

Broadly speaking, IMS can be applied for two functions: separation of ions and the measurement of arrival time distributions (ATDs) and calculation of CCS (structural applications) in the hope of defining precise gas-phase structural information from computational studies. However, there is currently no specific set of IMS conditions that have been demonstrated to be able to separate all carbohydrates or computational methods to generate theoretical structures. As a result, IMS separation of derivatized[106] and underivatized carbohydrates have been performed with a plethora of different adducts in both positive and negative ion mode.[107-110] Separation has been achieved with a variety of drift gases,[108, 111-112] at different temperatures and pressures, on different mobility analysers.[111, 113-114] Samples have also been ionized by different techniques, predominantly MALDI[110] and ESI,[114]with differing sample preparations, all of which will affect the measured ATDs. Improved separation has been reported in numerous ways for each IMS device. Changing the drift gas to larger, more polarizable molecules such as carbon dioxide has resulted in the ability to baseline separate isomers, which were otherwise unresolved.[108, 111, 115] Lalli et al. reported for other small molecule isomers that increasing the pressure also improves the resolving 
power (rather than peak-to-peak distance), although massively impacts on the ion transmission (i.e. sensitivity).[111] Raising the temperature of the drift gas is also likely to reduce the resolving power based on theoretical calculations, although no systematic study has been attempted for carbohydrates as far as the authors are aware.[116-117] Additionally it is (currently) impossible to discern any structural information from computational calculations using polarizable gases as no suitable algorithms have been formulated.[111] Alternatively, changing the ions adduct type can result in differential mobilities.[118] These factors have allowed the separation $\alpha / \beta$-anomers of 1 -Me-Glc, 1 Me-Gal and 1-Me-Man.[108] Recently, Hofmann and co-workers reported the greatest separation of anomers and regioisomers as deprotonated species compared to protonated, sodiated or chloride adducts.[107] Separation as metal adducts may be more challenging as the structures 'deform' in the gas-phase in order to solvate the charge of the adduct,[119] potentially masking isomeric differences in certain cases. Computational studies can also be more challenging when using more 'exotic' adducts (such as heavy metals) for reasons similar to those occurring with different drift gases. Improvements in IMS separation achieved by changing the drift gas or adduct are difficult to predict as they depend on the chemical properties of the analyte.[120-121] In fact the choice of adduct is normally dictated either by the analyte, (acidic carbohydrates normally deprotonate readily[122] whereas neutral carbohydrates tend to sodiate[114]) or its ability to generate rich, structurally informative and diagnostic product ions upon activation of the complex.[123-124] Applications of IMS to facilitate separation and structural characterization of glycans will be discussed in detail for the different types of glycans and glycoconjugates.

\subsection{Free-glycans}

\subsubsection{Mono- to hexa-saccharides}

\subsubsection{Separation}

Initial application of IMS to carbohydrates focused on whether or not IMS had sufficient resolution to distinguish simple (mainly mono-, di- and tri-saccharides) isomeric carbohydrates. Liu et al. (1997) first demonstrated that ability of DTIMS ${ }_{\mathrm{N} 2}$ to distinguish a small sub-set of isomeric precursor and product ions derived from raffinose and melezitose and $\alpha$-cyclodextrin, $\beta$-cyclodextrin and $\gamma$-cyclodextrin.[125] Using their home-built DTIMS ${ }_{\mathrm{N} 2}$ device, the Hill group then demonstrated separation of 21 different carbohydrate standards, including a significant number of isomers.[126] The same group later reported the ability to separate a substantial number of all known (sodiated) 1methyl hexoses and selected 1-methyl N-acetylhexosamines (GlcNAc and GalNAc) monosaccharides as both their $\alpha$ - $/ \beta$ - anomers using both DTIMS and TWIMS. For the most part $\alpha$ - and $\beta$ - 1 -methyl anomers of each hexose could be discriminated by both DTIMS and TWIMS other than gulose and certain diastereoisomers could also not be separated such as 1-Me- $\beta$-allose and 1-Me- $\beta$-glucose.[127] As might be expected the DTIMS displayed a higher resolving power. Recently, the Pagel and 
Seeberger groups demonstrated the ability of IM-MS to separate and quantify anomers and regioisomers of chemically synthesized tri-/penta-saccharide precursor and product ions down to concentrations as low as $0.1 \%$ of the dominant anomer glycans (Figure 8).[107] 

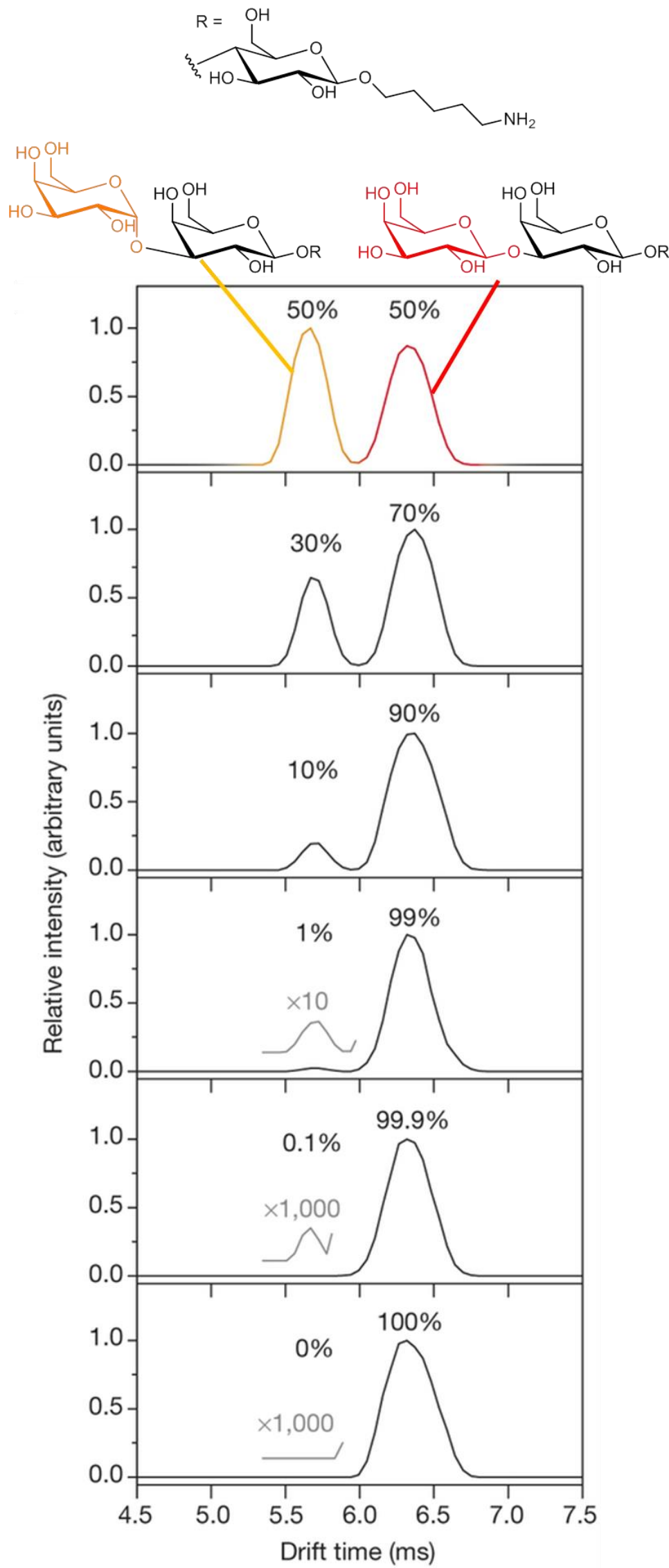

Figure 8 - ATDs associated with a mixture of two anomeric carbohydrates at ratios defined on each distribution. Figure amended from Hofmann 2015.[107] 
FAIMS has also been demonstrated to be capable of resolving anomeric, linkage and positional isomers of a series of chemically synthesized glycosides as a number of different adducts in both positive and negative ion mode.[109] Mobility separated precursors are routinely dissociated by collision-induced dissociation (CID) to generate structural information.[123, 128-133]

Even with application of IMS, baseline separation of isomeric precursors is often not fully achieved. Chimeric ATDs resulting from isomeric precursors can be extracted if a post-mobility generated product ion is specific to a given isomer (sometimes termed extracted fragment ion drift time distributions (XFIDTDs)).[118, 132] Post-mobility CID enabled elucidation of isomeric glycosides within the glycourinome of a patient suffering from symptoms assigned to a congenital disorder of glycosylation.[134] This structural variance could have been overlooked by MS alone. Alternatively, the mobility of product ions generated from CID of isomeric precursors may be separable.[118, 130, 135] For example Li et al. (2013) measured the ATDs of a series of dihexose anomers, regioisomers and diastereoisomers by TWIMS. Despite the fact that certain precursors coeluted in mobility, their respective monosaccharide-glycolaldehyde cross-ring product ions did not.[135] To circumvent the possibility of the quadrupole co-selecting isomeric/baric precursors for fragmentation, which would result in unwanted chimeric product ion spectra, the same group incorporated a dual gated DTIMS device in place of the source region of a Synapt HDMS (commercial TWIMS instrument). This allows mobility selection of precursors prior to mass selection, CID and subsequent mobility separation of the generated products ions (tandem IMS). This mobility selection was achieved using a dual gate drift tube where one gate allows ions to enter the drift cell and the second allows ions to leave the cell. The second gate is opened at a defined time after the first allowing only ions of that specific mobility to be transferred into the ion trap. However, this gating of ions dramatically reduces the duty cycle and by virtue the sensitivity (Figure 9).[136] Further improvements in separation has been achieved by combining energy-resolved (ER)-MS with IMS, since diastereomeric glycans may dissociate at different energies.[124, 137] 


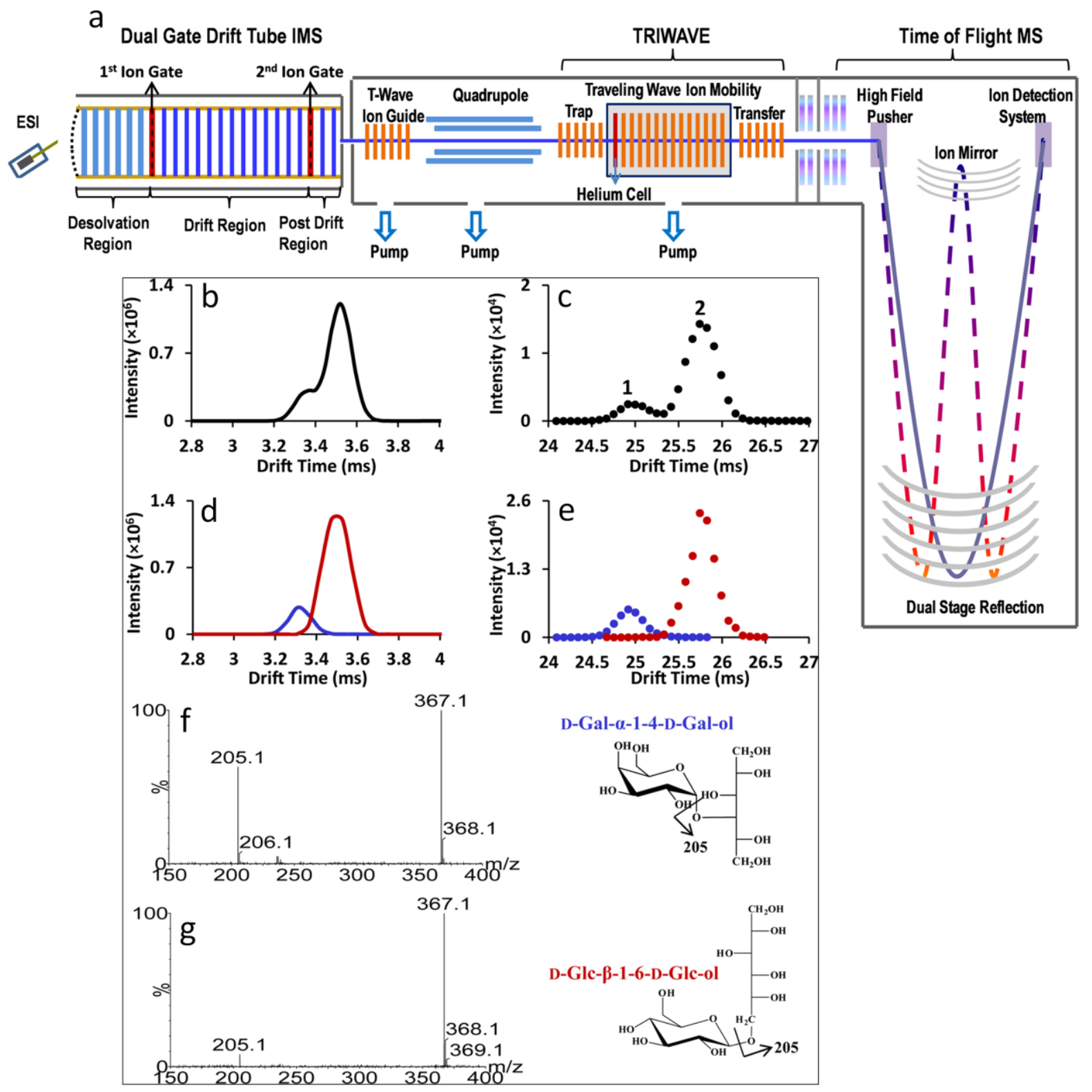

Figure 9 - The tandem IMS instrument developed by the Hill group, formed from connecting a dual gated DTIMS onto the source of a TWIMS mass spectrometer (a). Inset are the DT (b) and TW (c) ATDs associated with a mixture of Galo-1-4Gal-ol and Glcß1-6Glc-ol. ATDs of the pure compounds measured by the DT (d) and TW (e) analyser confirming that the mixture is separable. (f) and (g) are the tandem mass spectra associated with the mobility selected peaks labelled 1 and 2, respectively, within panel (c). Figure amended from $\mathrm{Li}$ et al.[136]

From initial work on simple chemically synthesized standards, isomeric disaccharide-alditols, chemically released from the $O$-linked glycoprotein bovine submaxillary mucin (BSM) by alkaline borohydride treatment, have been separated by IMS, which could not be achieved by HPLC alone. $[129,138]$ Unlike reducing carbohydrates these alditols possess less conformational variability (i.e. narrow ATDs consisting of a single peak), which is likely as a result of the inability of non-reducing glycans to mutarotate between isomeric structures.[128-129] $\mathrm{MS}^{\mathrm{n}}$ (multistage tandem mass spectrometry where $\mathrm{n}$ denotes the number of activation events) of mobility selected HPLC fractionated BSM carbohydrate-alditols, yielded further diagnostic structural information.[128-129] 
Anionic carbohydrate alditols were also separable by TWIMS with greater sensitivity, albeit at the loss of some IMS resolving power.[130] Post-mobility CID generated diagnostic tandem mass spectra from isomeric precursors. Additionally, CID generated BSM carbohydrate-alditol product ions could be separated with and without mass isolation. The mobility separated product ions could also be made to dissociate again producing further structural information.[130]

As the reader may have noticed, the vast majority of these strategies exploit CID to fragment precursors, principally because it is the simplest method to achieve dissociation. However, other informative dissociation methods have also been employed. Lee et al. performed vacuum ultraviolet photodissociation (VUVPD) $(157 \mathrm{~nm})$ on several mobility-separated isomeric disaccharides, which produce similar CID tandem mass spectra.[123] VUVPD generated significantly more cross-ring fragments than CID and their presence was found to be highly dependent on the regiochemistry and anomeric configuration of specific precursors. This enabled discrimination and identification of all isomers.[123] Finally Hernandez et al. mobility selected lithiated, sodiated and potassiated $\alpha / \beta-1-$ methyl anomers of Glc and Man by FAIMS.[131] Mobility selected ions were accumulated in a quadrupole ion trap before being irradiated with various wavelengths of infrared radiation. Ions absorb IR radiation and dissociate when their structures possess resonant vibrational modes. Therefore plotting the fragmentation yield against the wavelength of light, normalized to the respective laser power at each frequency, generates an action IR spectrum. From this action IR spectrum, the gasphase structures of each of these complexes were determined in the presence of a single water molecule (Figure 10).[131] The combination of IMS, IR and MS is an extremely powerful technique. IMS can separate isomers, which would be indistinguishable by MS alone and can provide structural information on the intact complex; MS rapidly separates species by their $\mathrm{m} / \mathrm{z}$ and can generate connectivity information through tandem mass spectrometry; IR provides specific atomistic information often lacking from IM-MS approaches alone. 


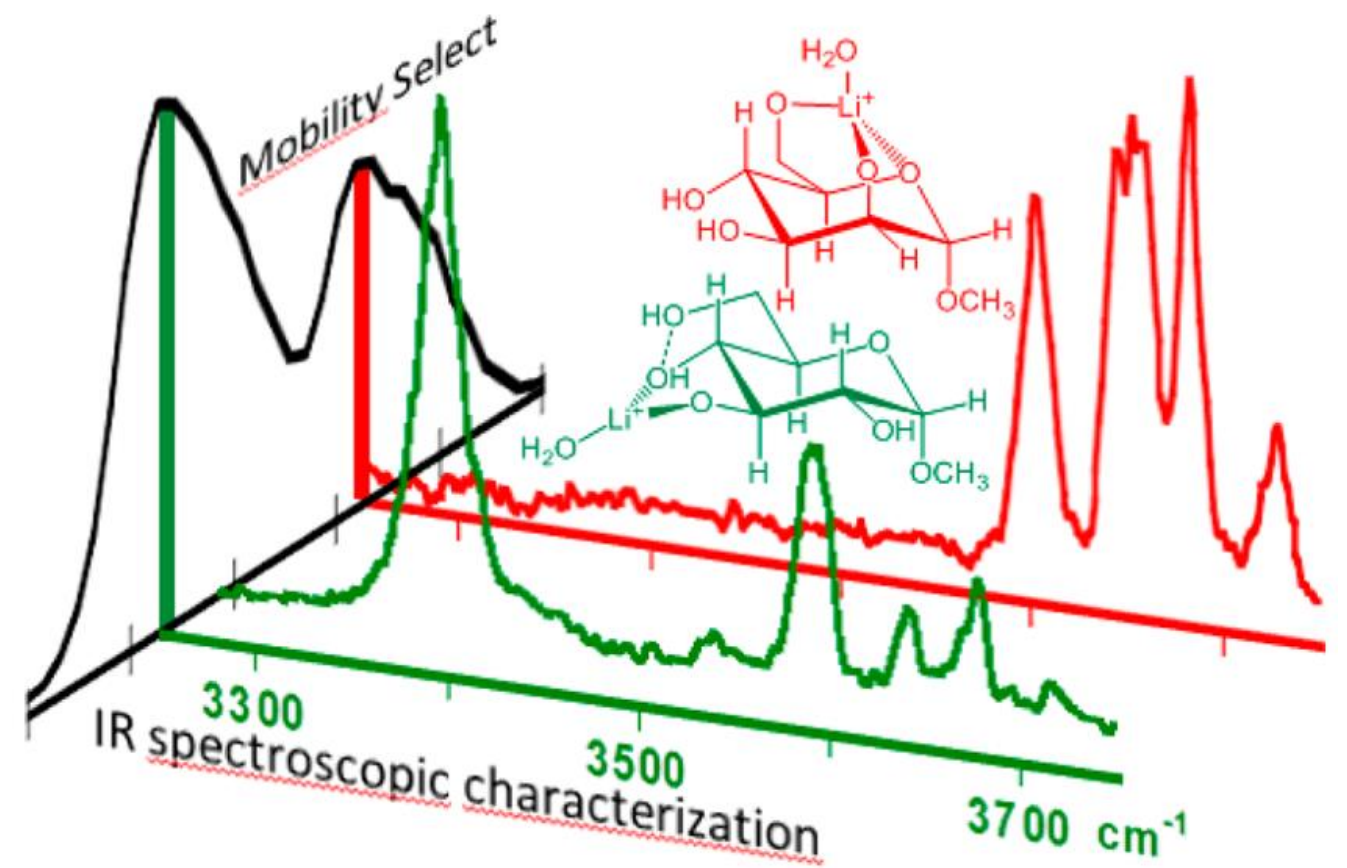

Figure $10-\left[\mathrm{Me \alpha 1}-\mathrm{Glc}+\mathrm{H}_{2} \mathrm{O}+\mathrm{Li}\right]^{+}$(green) and $\left[\mathrm{Me} \alpha 1-\mathrm{Man}+\mathrm{H}_{2} \mathrm{O}+\mathrm{Li}\right]^{+}$(red) complexes, mobility separated by FAIMS (black trace). Species can then be mobility selected prior to variable wavelength action IR MS. MD calculations of theoretical structures that would give rise to these traces suggest that the coordination site of $\mathrm{Li}^{+}$differs between Mea1-Glc and Mea1-Man. Figure taken from Hernandez.[131]

IMS provides benefits, other than just separating isomeric ions: it can be used to help 'filter' carbohydrate specific data from contaminating ions or other molecular species.[134] This can be easily visualized on a plot of $\mathrm{m} / \mathrm{z} v \mathrm{~s}$. drift time/CCS as certain classes of ions tend to run in similar regions on this plot. This enables simultaneous analysis of glycans and glycopeptides within the same experiment, circumventing laborious sample purification and separation steps and potentially facilitating future high-throughput glycoproteomics.[139] Carbohydrates can also be chemically derivatized to alter their $\mathrm{m} / \mathrm{z}$ and CCS, so they appear in an uncongested region within this $2 \mathrm{D}$ plot.[106]

\subsubsection{Structural}

As mentioned within the introduction of this section, IMS can provide structural information, facilitated by computational calculations. This was first reported by the Bowers group, who measured the ${ }^{\mathrm{DT}} \mathrm{CCS}_{\mathrm{He}}$ of a series of sodiated tetrahexoses and hexoses. The lowest energy conformations of these structures were computed and their theoretical $\mathrm{CCS}_{\mathrm{He}}$ determined by projection approximation. For each carbohydrate, the lowest energy structures had the carbohydrate chain wrapping around the sodium ion, forming 4 coordinate bonds to 4 monosaccharide units, solvating the charge. These theoretical $\mathrm{CCS}_{\mathrm{He}}$ matched well with experimental values suggesting that these were indeed reasonable representations of the measured gas-phase structures.(Figure 11)[119] 

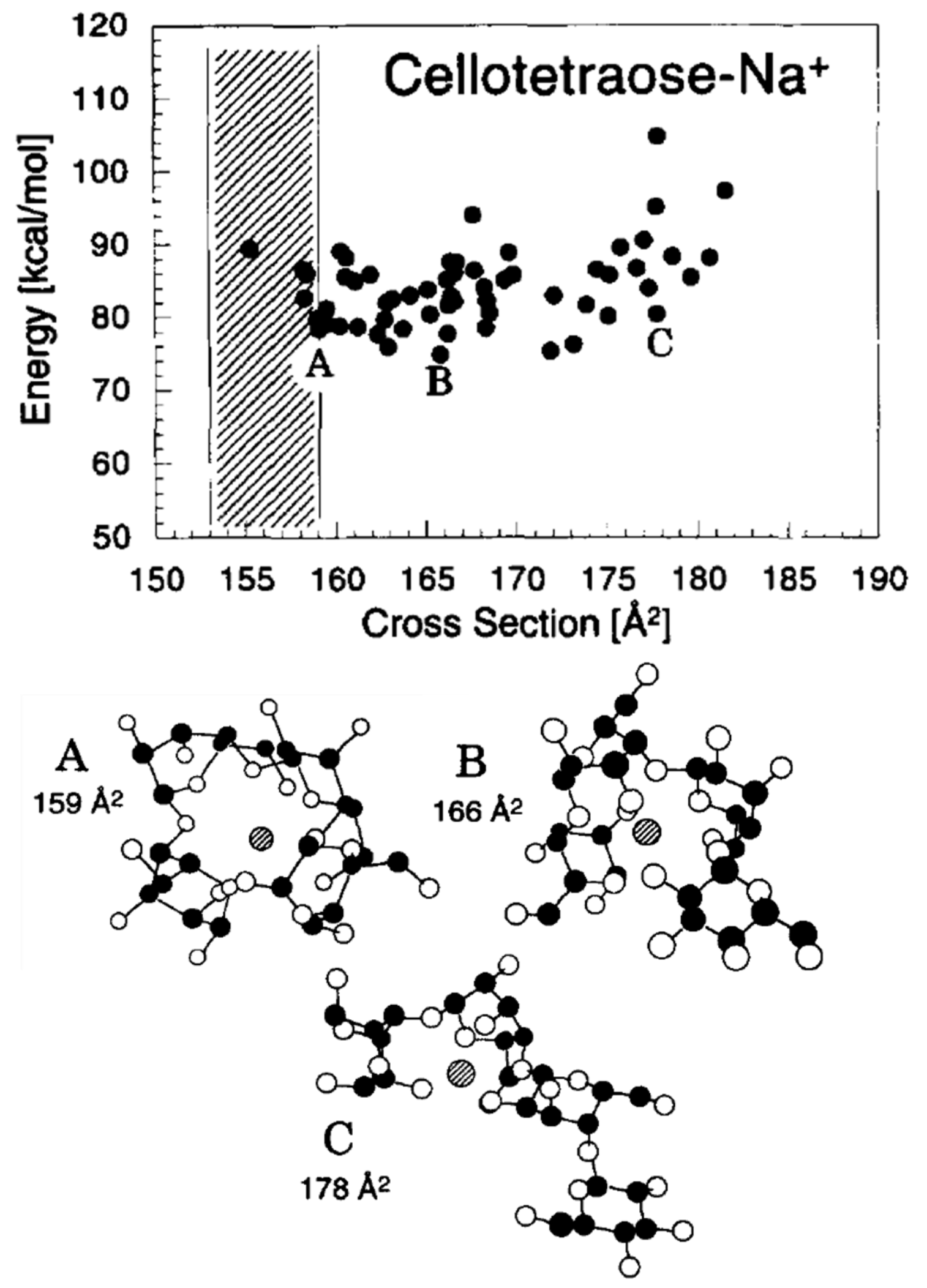

Figure 11 - Scatter plot representing the energy and cross section of MD generated sodiated callotetraose structures. The shaded area highlights the experimentally measured ${ }^{\mathrm{DT}} \mathrm{CCS}_{\mathrm{He}}$. Structures $\mathrm{A}, \mathrm{B}$ and $\mathrm{C}$ are representative structures within areas of the plot, Figure amended from Lee $e t$ al.[119] 
Leavel et al. showed by DTIMS that for a $[\mathrm{Zn}+\text { diethylenetriamine+hexose }+\mathrm{Cl}]^{+}$complex the stereochemistry of the hexose also influences the metal binding site.[120] A significant number of recent papers have employed TWIMS to measure the CCS of carbohydrate ions. As mentioned in section $2.2 \mathrm{CCS}$ measurements can only be made on TWIMS instruments by comparing it to a calibration curve of standards whose CCS areas had been previously measured on a DTIMS instrument. However, care must be taken when choosing appropriate standards as it has been reported that calibrating glycan CCS to a peptide calibrant introduces a small but systematic error of $\sim 1 \%$, presumably due to differences in the interaction potential between peptides and carbohydrates with the drift gas.[74-75] Similarly, charge state mismatching introduces large errors ( $3.5 \%)$, i.e. the error associated with normalising both mass and charge states within the measured data. Mismatching both charge state and the molecular class increases this error further ( 4.7\%).[74] Similarly, large errors are associated with trying to convert ${ }^{\mathrm{DT}} \mathrm{CCS}_{\mathrm{He}}$ to ${ }^{\mathrm{TW}} \mathrm{CCS}_{\mathrm{N} 2}$. [75] Fenn et al. measured the ${ }^{\mathrm{DT}} \mathrm{CCS}_{\mathrm{He}}$ values for over 300 carbohydrates including isomers, [118] and Hofmann et al. measured the ${ }^{\mathrm{DT}} \mathrm{CCS}_{\mathrm{He}}$ and ${ }^{\mathrm{DT}} \mathrm{CCS}_{\mathrm{N} 2}$ of a dextran polymer[75], both of which can be used to calibrate TWIMS analysed carbohydrates. Pagel et al. also determined the ${ }^{\mathrm{DT}} \mathrm{CCS}_{\mathrm{He}}$ and ${ }^{\mathrm{DT}} \mathrm{CCS}_{\mathrm{N} 2}$ of $\mathrm{N}$-glycan precursors and product ions. Glycans were released with hydrazine from RNase B, fetuin, asialofetuin, ovalbumin, porcine thyroglobin and desialylated porcine thyroglobin, which could be beneficial for calibration of TWIMS for N-glycan analysis as discussed within the next section.[140]

Huang et al. measured the ${ }^{\mathrm{TW}} \mathrm{CCS}_{\mathrm{N} 2}$ of various di-, tri-, penta- and hexa-saccharides as their group I metal adducts $\left(\mathrm{Li}^{+}, \mathrm{Na}^{+}, \mathrm{K}^{+}, \mathrm{Rb}^{+}, \mathrm{Cs}^{+}\right)$and noted that the ${ }^{\mathrm{TW}} \mathrm{CCS}_{\mathrm{N} 2}$ generally increased as the metal ion radius increased, although in an isomer specific manner, which likely reflects the differences in coordination site.[118, 121] The same group also demonstrated IMS separation of isomeric lacto-N-fucopentose I and lacto-N-fucopentose V, and lacto-N-difucohexaose I and lacto$\mathrm{N}$-difucohexaose II as $\mathrm{Ca}$ and $\mathrm{Ba}$ radical cation adducts,[141] which have been reported to be difficult to separate by both IMS and $\mathrm{MS}^{2}$, including as divalent $\mathrm{Ca}^{2+}$ and $\mathrm{Ba}^{2+}$ adducts.[118, 121, 141-143] An additional benefit was that these radical cations generated much more diagnostic CID product ions compared to their divalent equivalent.[141]

Structure determination is not only limited to precursor ions, but also product ions. This is exemplified by the work of Both et al. who measured the TWIMS arrival time distributions of a series of isomeric hexose and $\mathrm{N}$-acetylhexosamine glycosidic product ions (B-, C-, Z- and $\mathrm{Y}$-) derived from CID of either glycopeptides or reducing carbohydrates.[114] Crucially, the ATDs of these product ions were independent of the initial precursor, therefore they were able to 'sequence' the stereochemistry of the monomeric product ions (Figure 12). Incorporation of multistage tandem mass spectrometry prior to IMS enabled complete elucidation of the monosaccharide building blocks within Man3 glycan and helped reveal promiscuity of the glycosyltransferase pp-GanT2.[114] 


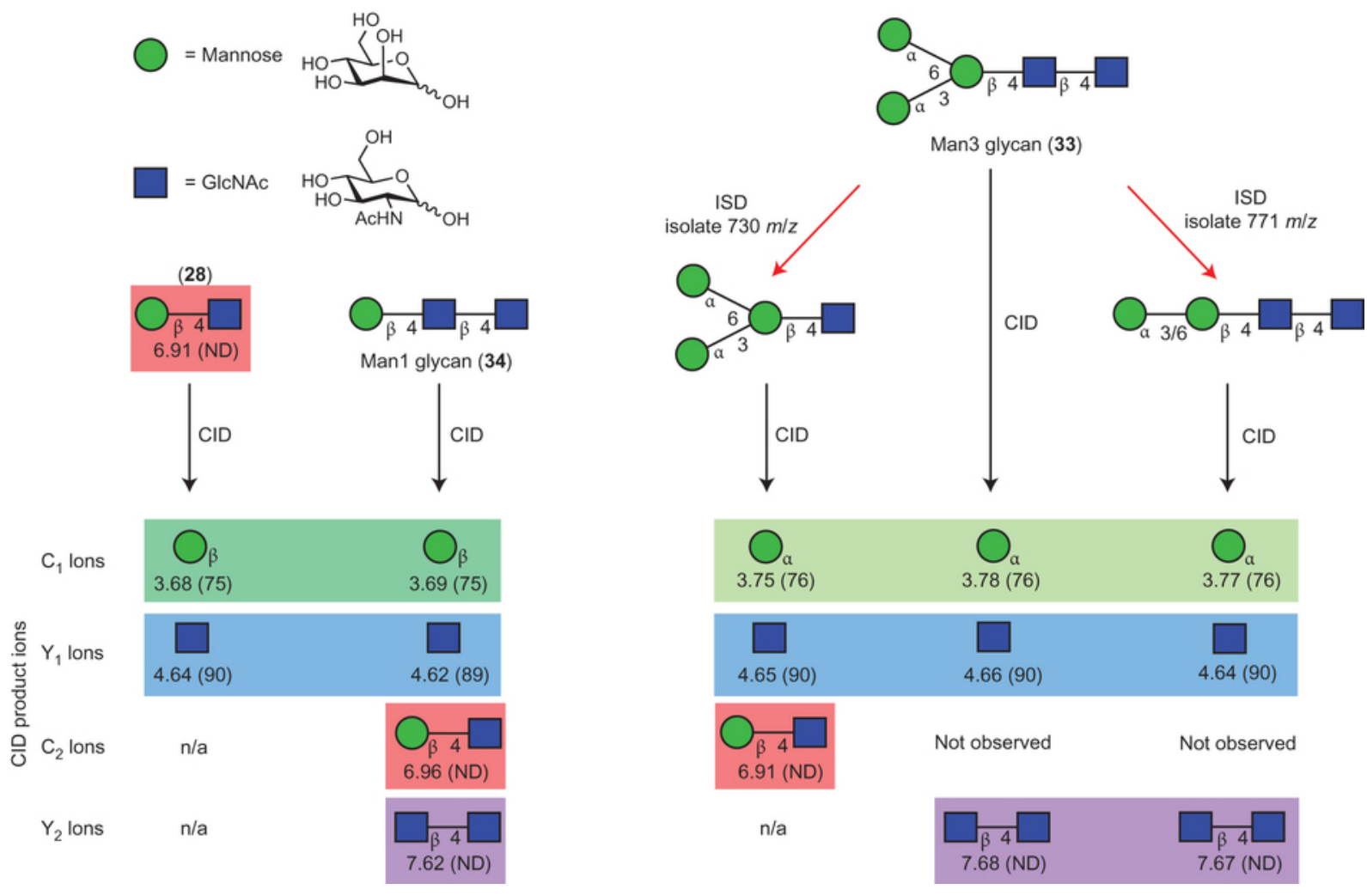

Figure 12 - Drift times (in ms) and CCS values (in $\AA^{2}$ ) in parentheses for selected [M+Na] $]^{+}$product ions are presented below the structures. Terminal monosaccharide and disaccharide product ions could be characterized after a single round of CID. Internal residues were characterized following in-source dissociation (ISD), quadrupole mass isolation of the desired ISD product ion, and subsequent CID before IM-MS. $\mathrm{C}_{1}$ ions can be grouped either as $\beta$-Man (shaded dark green) or $\alpha$-Man (shaded light green). All identified $Y_{1}, C_{2}$ and $Y_{2}$ product ions could be characterized as GlcNAc (shaded blue), Manß1-4GlcNAc (shaded red) and GlcNAcß1-4GlcNAc (shaded purple), respectively. For cases where two conformations (potential sites of sodiation) were observed, the drift time and CCS measurements are reported for the predominant species. CCS values for the disaccharides were not determined (ND) as the drift time values lie outside this calibration range. n/a, not applicable. Figure taken from Both et al.[114]

Recently, the Clemmer group measured the ${ }^{\mathrm{DT}} \mathrm{CCS}_{\mathrm{He}}$ of lithiated precursor and CID generated product ions on a home-built tandem DTIMS instrument. Even though specific precursors had identical CCS (for example sucrose and leucrose) their product ion CCSs differed (although these product ions may have consisted of multiple different species due to the potential existence of isomeric product ions).[144]

\subsubsection{N-glycans}

Analysis of structurally larger and more complicated N-glycans largely built upon the initial studies on smaller glycan standards.[139-140] Fenn et al. and Harvey et al. demonstrated the benefit of the additional gas-phase separation afforded by IMS to help characterize $N$-glycan isomers released from the glycoproteins RNase B, gp120 and fetuin, either enzymatically or chemically.[110, 122, 139] Zhu and co-workers were able to separate four isomers of permethylated Man7 glycan released from RNase B by DTIMS and post-mobility CID. Deconvolution of the ATDs into the four isomers was facilitated by extracting the ATDs associated to isomer specific fragment ions.[132] Like for small molecules, N-glycans can also be readily separated from contaminating species. [122, 145-146] For 
example $\mathrm{N}$-glycans have been profiled directly following PNGase F release in the presence of salt and detergents. This minimizes sample loss from purification strategies or liquid handling steps and additionally reduces the experiment time. $[122,146]$ Glycosides can also be readily deconvoluted into classes from the $m / z v s$. drift time 2D plots (Figure 13), for example singly, doubly and triply charged glycans and glycopeptides. These species would be unidentifiable based on the signal-to-noise of the MS acquisition alone.[122] Glycans and glycopeptides could also be fractionated by LC prior to IMS to improve separation and the detection capacity.[145]

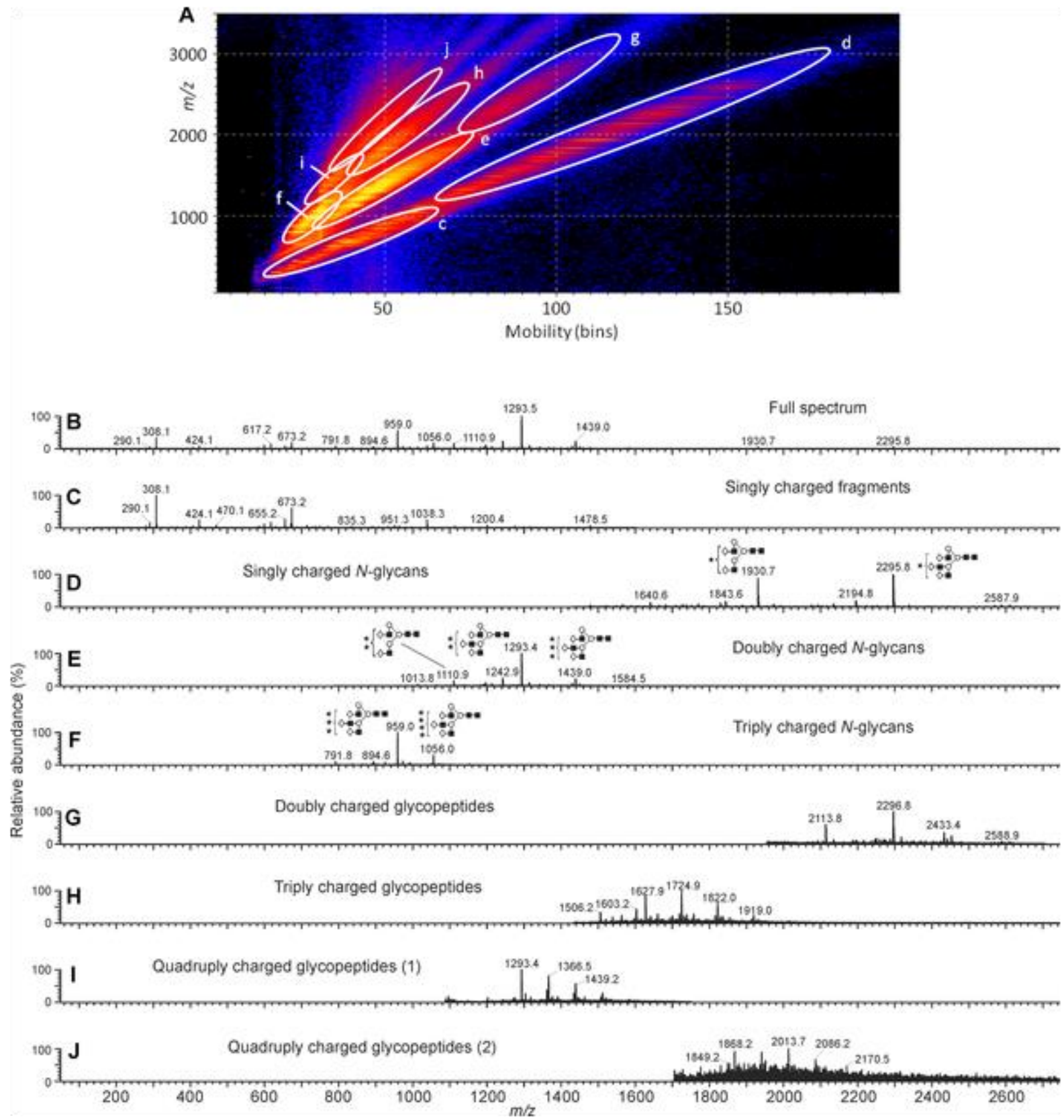

Figure 13 - 2D plot of the drift time against $\mathrm{m} / \mathrm{z}$ (log intensity scale) of the negative charged ions generated from ESI of $\mathrm{N}$-glycosides released from bovine fetuin by hydrazinolysis (a). The total mass spectrum (b) and the extracted mass spectra of the regions (c-j) within the 2D plot. Figure taken from Harvey et al. with permission.[122] 
With the additional orthogonal separation afforded by IMS, Isailovic et al. demonstrated profiling of total human serum glycans from patients suffering from liver cancer or cirrhosis of the liver.[26] Differences in the ATDs associated to specific permethylated N-glycan precursor ions, likely result from changes in the isomeric distribution of that given ion. In some cases, variance in the ATDs of certain glycan ions between individuals could be separated into healthy and diseased cohorts after principle component analysis (PCA).[26] This data suggests that aberrations in isomer distributions may be indicative of disease states. Considering ATDs associated with multiple different isomeric glycans improved the reliability of the PCA to cluster 81 individual samples into specific disease (and apparently healthy) cohorts significantly (Figure 14),[27] whereas MS alone did not demonstrate any significant clustering. Using the same strategy, Gaye and co-workers were able to characterize serum N-glycans into cohorts derived from three disease phenotypes namely Barrett's esophagus, high-grade dysplasia and esophageal adenocarcinoma, from normal controls of apparently healthy individuals.[28] Interestingly, a significant number of glycans selected for PCA within this experiment were identical to those selected for the liver disease state experiments.[28]

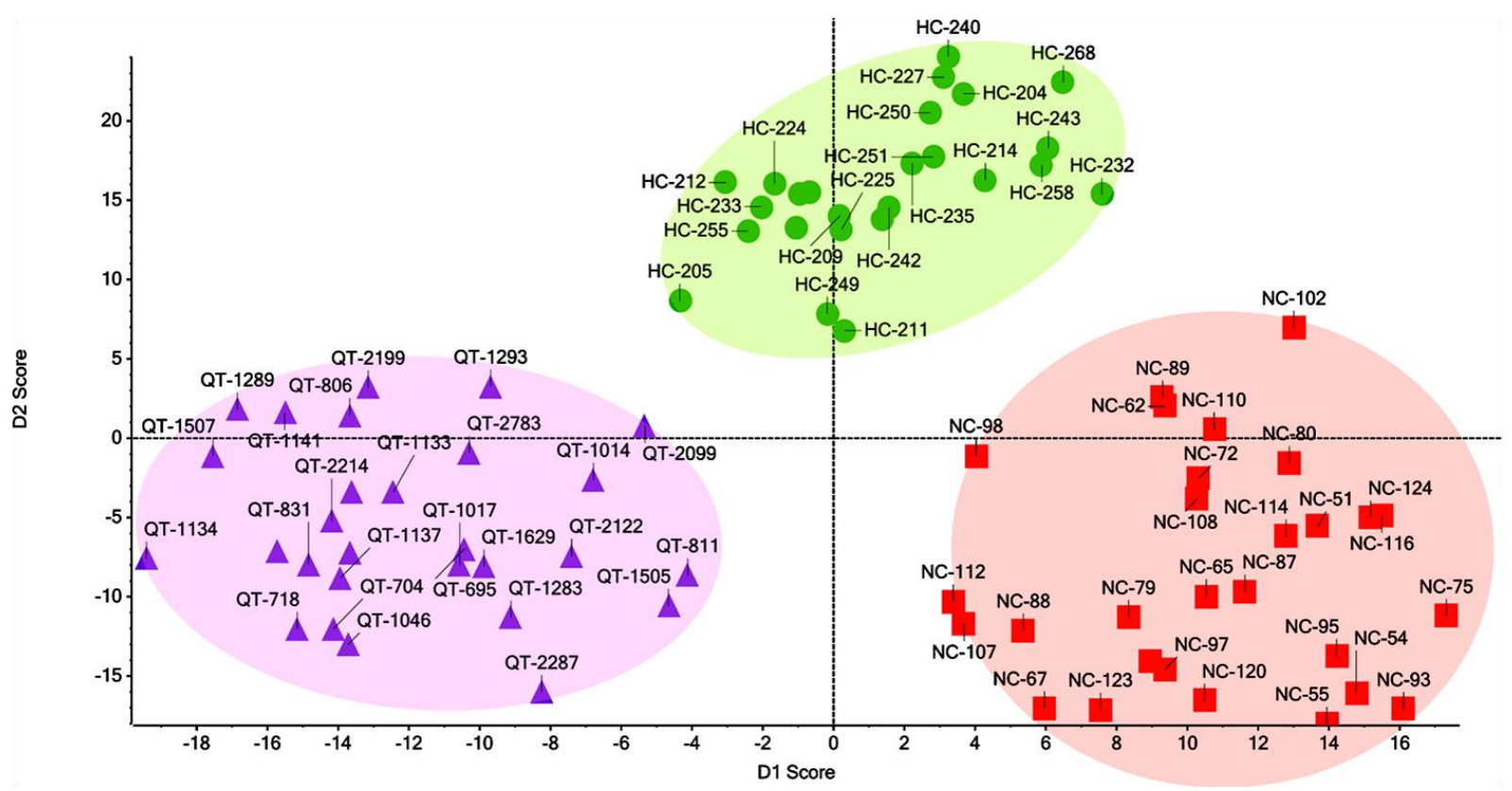

Figure 14 - Supervized PCA of drift time profiles of 10 unique $m / z$ glycan ions namely Fuc $_{1} \mathrm{Hex}_{5} \mathrm{HexNAc}_{4}{ }^{3+}$, Neu5Ac $_{1} \mathrm{Hex}_{5} \mathrm{HexNAc}_{4}{ }^{2+}$, Neu5Ac $\mathrm{Hex}_{5} \mathrm{HexNAc}_{4}{ }^{3+}, \mathrm{Neu5Ac}_{1} \mathrm{Fuc}_{1} \mathrm{Hex}_{5} \mathrm{HexNAc}_{4}{ }^{{ }^{+}}, \mathrm{Neu5Ac}_{2} \mathrm{Hex}_{5} \mathrm{HexNAc}_{4}{ }^{2+}$,

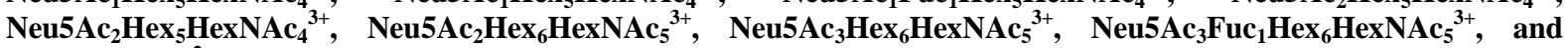
$\mathrm{Hex}_{5} \mathrm{HexNAc}_{2}{ }^{2+}$ found within human serum. NC, HC and QT are serum samples from apparently healthy individuals, those with hepatocellular carcinoma and those with cirrhosis respectively. Taken from Isailovic et al.[27]

Given the increased structural complexity associated with N-glycans compared to the smaller glycosides, few papers have been able to deliver structural information from the IMS data. Impressively though, Plasencia et al. determined the ${ }^{\mathrm{DT}} \mathrm{CCS}_{\mathrm{He}}$ for three species associated with the disodiated permethylated $\mathrm{Hex}_{5} \mathrm{HexNAc}_{4}$ glycan released from ovalbumin (Figure 15). They argue that these three species arise from three different isomers of $\mathrm{Hex}_{5} \mathrm{HexNAc}_{4}$. CCS calculations (using TM) of the 10 lowest energy structures (after sequential simulated annealing) for each potential isomer 
were within $2.2 \%$ uncertainty of the experimental data, allowing assignment of each species within the ATD for these three isomers.[147] Williams et al. also determined the ${ }^{\mathrm{DT}} \mathrm{CCS}_{\mathrm{He}}$ and ${ }^{\mathrm{TW}} \mathrm{CCS}_{\mathrm{N} 2}$ for $\mathrm{N}$-glycans released from RNase B, ovalbumin and porcine thyroglobin with and without desialylation. Using both the EHSS and TM, they determine the CCS of modelled structures, which again match well with the experimental data from both DTIMS and TWIMS analysers.[79] Finally, Struwe et al. also measured ${ }^{\mathrm{DT}} \mathrm{CCS}_{\mathrm{He}},{ }^{\mathrm{DT}} \mathrm{CCS}_{\mathrm{N} 2},{ }^{\mathrm{TW}} \mathrm{CCS}_{\mathrm{He}}$, and ${ }^{\mathrm{Tw}} \mathrm{CCS}_{\mathrm{N} 2}$ for a series of $\mathrm{N}$-glycans as protonated, deprotonated, sodiated, potassiated, chloride and phosphate adducts. Computational simulations suggest that $\mathrm{N}$-glycans wrap around metal adducts to solvate charge, as previously demonstrated by the Bowers group for smaller carbohydrates.[119] Also for given carbohydrate ions, multiple conformations were observed. These CCS values were stored in a database (Glycomob housed within UniCarbKB) as a Glycomics IMS-MS resource.[148]

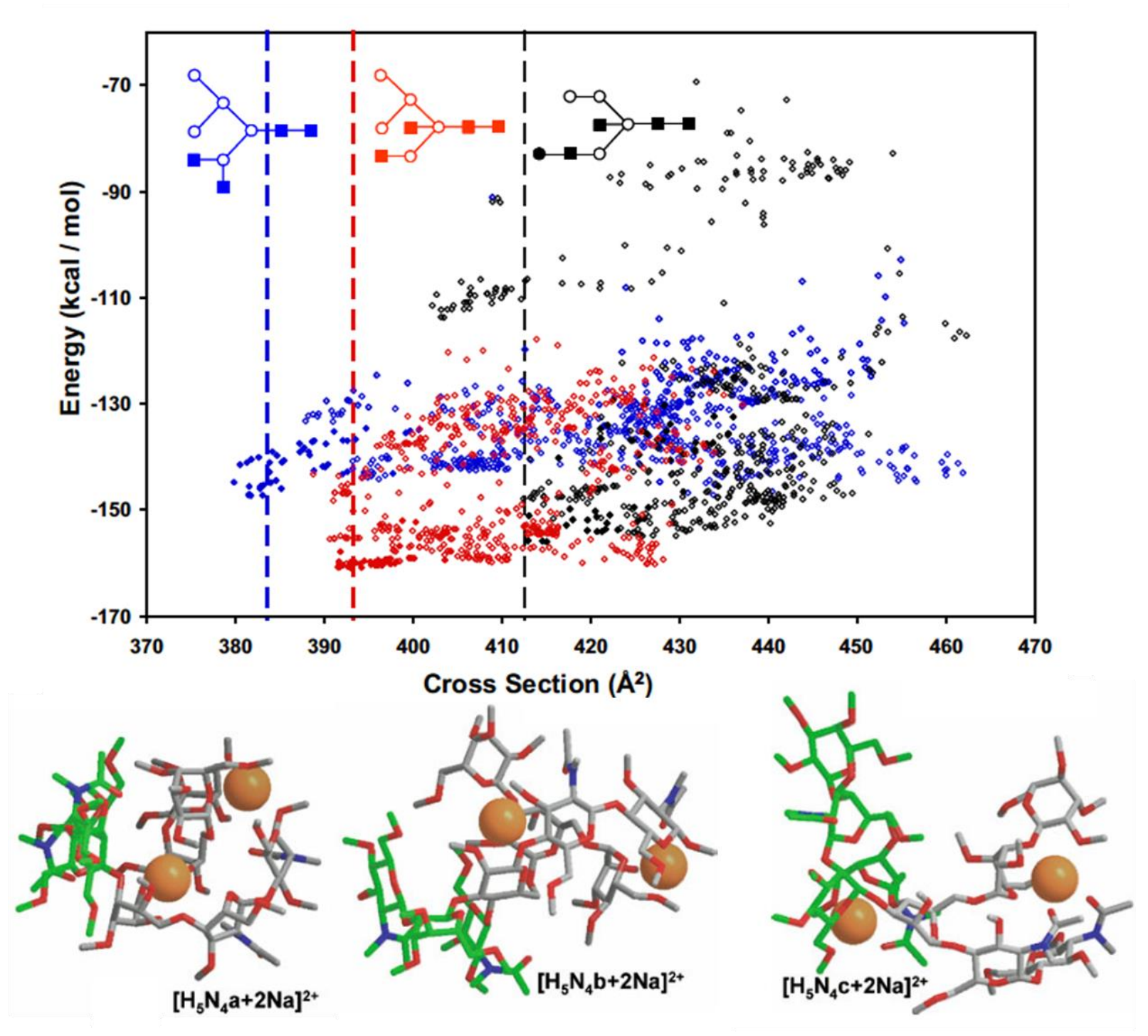

Figure 15 - Calculated cross sections (TM) and energies from MD simulations after simulated annealing for the three $\left[\mathrm{H}_{5} \mathrm{~N}_{4}+2 \mathrm{Na}\right]^{2+}$ isomers. Experimental CCS for the three isomers are highlighted by the dashed lines. $\left[\mathrm{H}_{5} \mathrm{~N}_{4} \mathrm{a}+2 \mathrm{Na}\right]^{2+}$, $\left[\mathrm{H}_{5} \mathrm{~N}_{4} \mathrm{~b}+2 \mathrm{Na}\right]^{2+}$, and $\left[\mathrm{H}_{5} \mathrm{~N}_{4} \mathrm{c}+2 \mathrm{Na}\right]^{2+}$ are represented by blue, red and black diamonds respectively. Representative 
low-energy structures for each isomer are shown. Bisecting GlcNAc and the $\alpha 1-3$ mannose arm are shown in green for clarity. Figure amended from Plasencia et al. ${ }^{[147]}$

\subsubsection{Glycosaminoglycans (GAGs) and other Polysaccharides}

Glycosaminoglycans (GAGs) are a family of linear anionic polysaccharides found almost ubiquitously on animal cell surfaces and within the extracellular matrices. GAGs have several biological functions such as cell-cell communication, cell adhesion, modulation of axonal and cell growth, proliferation, angiogenesis, anti-coagulation properties, viral invasion, tumor progression and metastasis.[149-153] Furthermore, they are known to bind numerous proteins such as chemokines, cytokines, proteases, growth factors, pathogen proteins and adhesion molecules.[150-151, 154-156] Consequently, GAGs have an extensive medical potential application (e.g. heparin for their anticoagulant properties).[157] Characterization of this family of polysaccharides is therefore fundamental to understand their structure/function relationship but represents a challenging task due to their inherent sequence, de-acetylation and sulfation heterogeneity.[30, 149, 154, 158-160] Consequently, despite the numerous biological activities of GAGs, there are few function specific sequences that have been completely elucidated.[161] It is also known that modification of the GAG affects its conformation and as a result influences its ability to bind certain proteins such as the fibroblast growth factor-2 or antithrombin that are involved in the blood-coagulation cascade.[162163] IMS offers the ability to monitor potential dynamic conformational changes upon varying the GAG sequence identity. Jin at al. performed conformational analysis of fully or partially sulfated heparin-derived di- and tetra-saccharides using a combination of DTIMS-MS and molecular modelling (using PA model) of ${ }^{\mathrm{DT}} \mathrm{CCS}_{\mathrm{He}}$.[81] The authors demonstrated that even minor changes to the primary structure of the GAG saccharides, for example changing the position of one sulfate or epimerization of an IdoA residue to GlcA, led to significant changes in conformation. Computational models supported the experimental results, and suggested that sodium adducts localized towards negatively charged groups and thus play an important role in conformation stabilization and the observed oligomerization of disaccharide units.[81] The heparin-derived tetrasaccharides wrap around the sodium cations, presumably to solvate charge, forming compact structures.[119] More recently, Leary et al. explored the affect various alkali metal $\left(\mathrm{Na}^{+}, \mathrm{K}^{+}\right)$, alkaline earth metal $\left(\mathrm{Mg}^{2+}, \mathrm{Ca}^{2+}\right)$ and transition metal $\left(\mathrm{Mn}^{2+}, \mathrm{Co}^{2+}, \mathrm{Fe}^{2+}, \mathrm{Ni}^{2+}\right)$ adducts had upon the conformation of a heparin octasaccharide,[164] since it has been previously demonstrated that these cations have influenced several protein-heparin interactions.[165-171] In all cases, increased affinity resulted in contraction of the heparin structure. However, conformational changes upon successive addition of metal cations depended on the identity of the metal adduct, which probably results from differing atomic radii and valences (Figure 16). In particular, the $\mathrm{Ca}^{2+}$ ion induced significant conformational contraction. Importantly, similar conformational changes were observed in solution to the gas phase IM-MS 
experiments, illustrating the relevance of the IMS techniques to investigate the structure/function relationship of GAGs.[164]
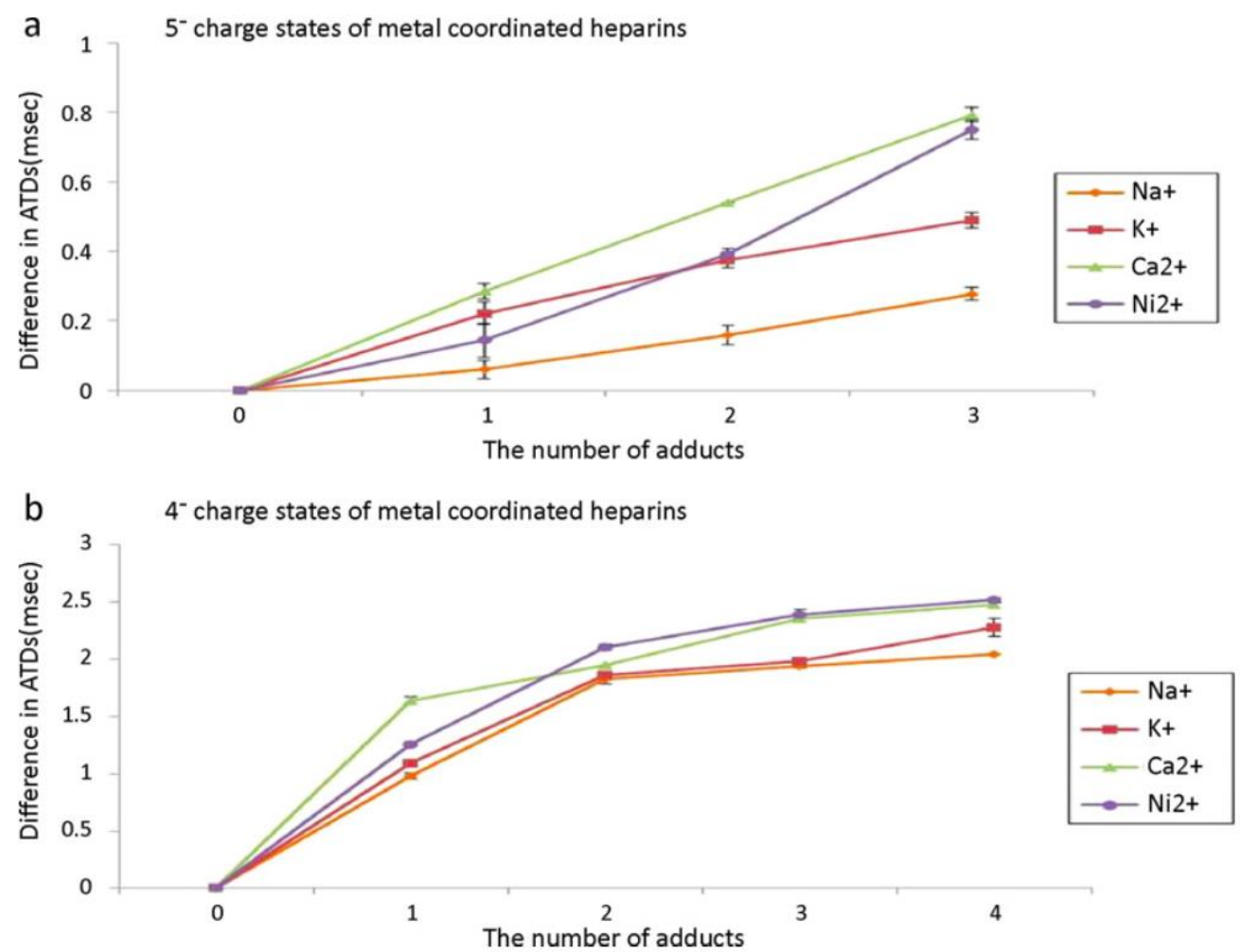

Figure 16 - The difference in arrival time distributions between metal and metal free coordinated heparin octasaccharide, plotted against the number of metal adducts (a) in the 5- charge state and (b) in the 4- charge state. Differences in ATDs are calculated by subtracting the ATDs for metal coordinated octasaccharides from those of the metal free species. ATDs of calcium and potassium coordinated octasaccharides that exhibit two ion populations in the ion mobility spectrum are shown with the most abundant ion population represented. Figure taken from Seo et al.[164]

Along with monitoring the conformational dynamics of GAGs, IMS has been used to separate isomeric species. The Leary group have reported applications of TWIMS-MS ${ }^{2}$ for the separation and the sequencing of two heparan sulfate (HS) isomers, facilitated by ${ }^{1} \mathrm{H}$ NMR.[172] Two isomeric HS hexasaccharides, purified by size exclusion and strong anion-exchanged chromatography, both produced highly similar mass spectra and enzymatic digestion profiles. TWIMS separation and subsequent CID of mobility separated ions was able to distinguish these epimeric hexasaccharides, which differed only by the stereochemistry at a single position (GlcA vs IdoA). Miller et al. also demonstrated TWIMS separation and identification of six tagged DP 8(degree of polymerization) heparan sulfate isomers differing only by their presence and absence of IdoA and GlcA at the hexuronic positions.[173] Fully IdoA DP8 structures displayed a more extended and flexible conformational distribution compared to the fully GlcA octasaccharide. The authors suggest this is because GlcA is reported to preferentially adopt the rigid ${ }^{4} \mathrm{C}_{1}$ chair conformation, whilst IdoA adopts a dynamic mixture of ${ }^{1} \mathrm{C}_{4},{ }^{4} \mathrm{C}_{1}$ and ${ }^{2} \mathrm{~S}_{0}$ conformations.[174-176] Interestingly, changes of IdoA to 
GlcA and vice versa within specific locations along the DP8 chain caused conformational distortions, which could be separated by TWIMS (Figure 17).
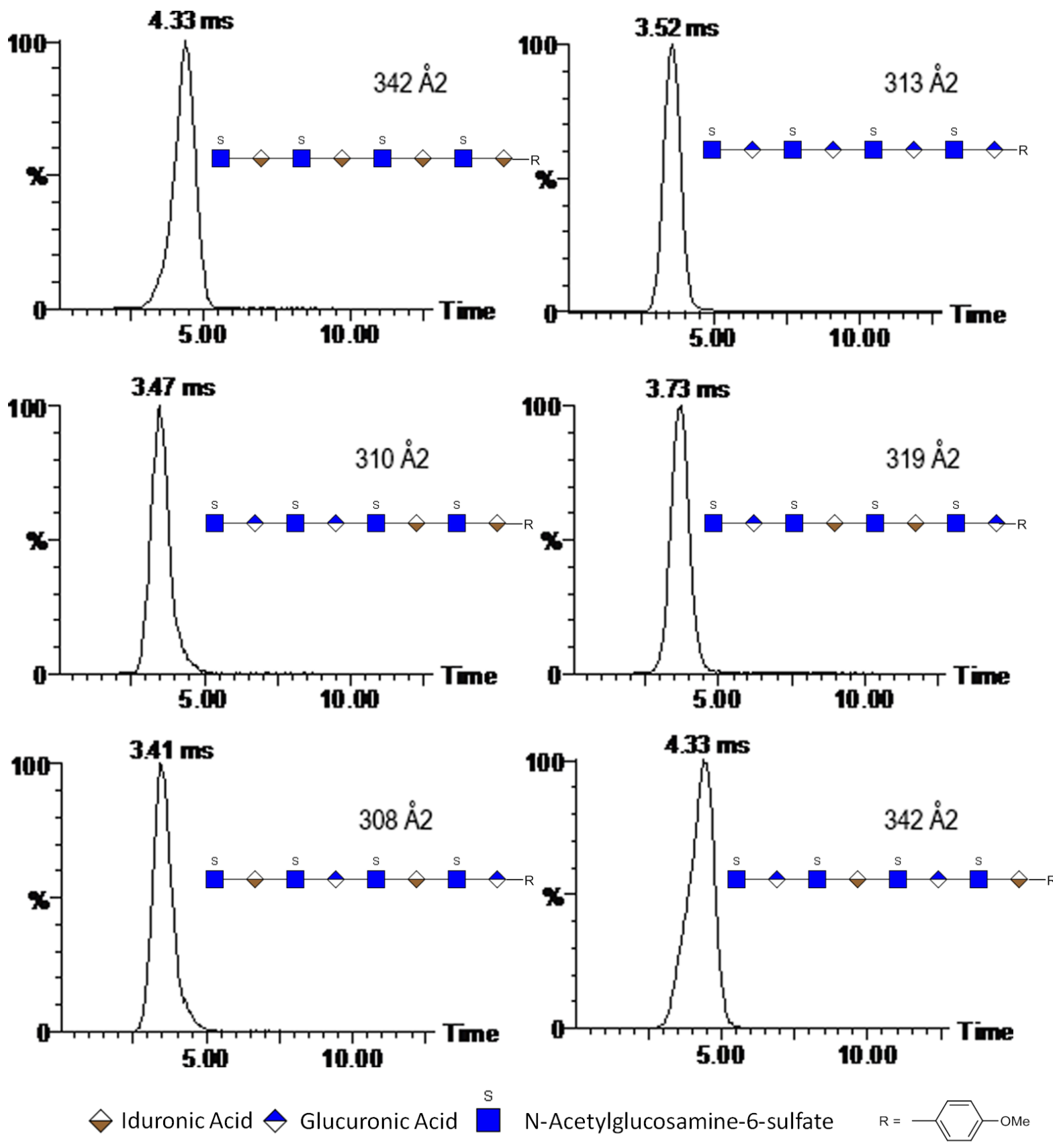

Figure 17 - Arrival time distributions and ${ }^{\mathrm{Tw}} \mathrm{CCS}_{\mathrm{N} 2}$ associated with six DP8 hexuronic acid positional isomers. Amended from Miller $e t$ al.[173]

The intensity distribution of specific fragments differed when GlcA or IdoA was adjacent to the glycosidic bond cleavage site (more facile for GlcA) allowing unambiguous elucidation of the hexuronic acid sequence. Another report described the ability of IM-MS ${ }^{2}$ to separate three synthetic heparin octasaccharides differing in sulfation or acetylation patterns employing a similar strategy.[177] Two arrival time populations were observed for certain oligosaccharides, suggesting the existence of two distinct conformations bearing the same sulfation pattern. Indeed, the presence of 
sulfation at the $2-\mathrm{OH}$ position of iduronic acid residues has been reported to lead to a mixture of conformers, corresponding to either a chair ${ }^{1} \mathrm{C}_{4}$ or/and a skew ${ }^{2} \mathrm{~S}_{0}$ conformation.[178] Recently, Lemr and co-workers reported the use of TWIMS combined with $\mathrm{MS}^{2}$ in order to separate hyaluronic acid isomers as well as to determine both their structural composition and their isomeric ratio.[179] While IMS allowed separation of tyramine positional isomers, subsequent CID fragmentation of separated precursor ions led to similar mass fragments often with only differing intensities, making structural characterization challenging. To overcome this limitation, the reducing end of the GAG isomers were reductively labelled by $\mathrm{NaBH}_{4}$ or stable isotope $\mathrm{NABD}_{4}$ (both performed in $\mathrm{H}_{2} \mathrm{O}$ ), which increased the mass by 2 or $3 \mathrm{~m} / \mathrm{z}$ respectively. This chemical modification resulted in the production of characteristic MS/MS fragments for each positional isomer and thus allowed their unambiguous identification and quantification even from mixtures.

Recently, Kailemia et al. have reported the first application of FAIMS, combined with Fourier transform ion cyclotron resonance mass spectrometry (FTICR-MS) and electron detachment dissociation (EDD) tandem MS, to mobility separate, select and characterize both anomeric and isobaric GAGs.[180] A chondroitin sulfate A (CSA) mixture of oligomers at various degrees of polymerization (DP4 to DP10), which was prepared by partial enzymatic digestion, produced similar MS spectra. FAIMS was able to separate the mixture of oligomers at different degrees of polymerization, although each peak was not necessarily baseline resolved. Furthermore, some oligomers were observed in more than one peak, which was attributed to the presence of a mixture of reducing anomers. Synthetic HS tetramers, whose reducing anomeric alcohol had been methylated, locking the anomer as either $\alpha$ - or $\beta$-, both produced a single distinguishable ATD under identical FAIMS conditions suggesting this hypothesis may be true. Alternatively, chemical reduction of the free reducing ends using $\mathrm{NaBH}_{4}$ to a single alditol species produced similar results. Mobility selected ions were fragmented by EDD, which is reported to preserve labile sulfate moieties, ${ }^{[181]}$ and $\mathrm{m} / \mathrm{z}$ of the resultant product ions were measured on an FTICR-MS for high mass resolving power. While the fragment ions obtained in every sample were similar, different intensities were observed. PCA allowed differentiation of each of the stereoisomers. ${ }^{[180]}$

Biologically relevant polysaccharides other than GAGs have also been studied by IMS to help separate isomers or monitor conformational variability. Rashid et al. have reported the discrimination of isobaric linear $\alpha$-glucans with a DP of up to 35 using TWIMS in negative ion mode.[80] Several singly charged $\alpha 1$-4-linked maltooligosaccharides and $\alpha 1-6$-linked dextrans, with DP comprised between 2 and 10, were distinguishable by their ${ }^{{ }^{T w}} \mathrm{CCS}_{\mathrm{N} 2}$. Similarly, doubly charged and triply charged equivalents of the species between DP 3-24 and 14-35 respectively, were also distinguishable (Figure 18). 

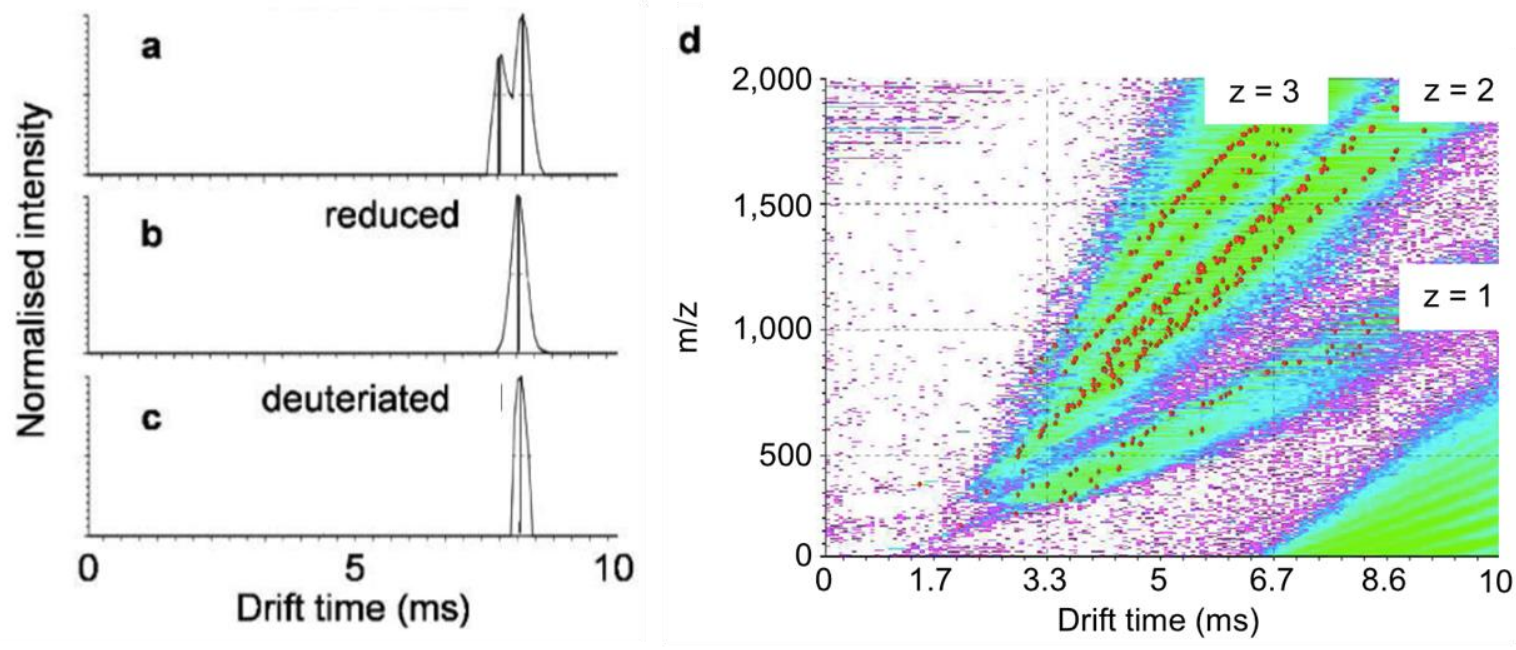

Figure 18 - Arrival time distribution associated with maltohexaose (a). The two species are thought to arise from a linear conformer and a V6 helical structure. After reduction (b) or deuteration (c), the conformational family reduces to a single, presumably disordered, species. Plot of drift time against $\mathrm{m} / \mathrm{z}$ of a mixture of maltooligosaccharides dextran 5000 and amylose 2800 (maltooligosaccharides) showing singly, doubly and triply charged ion clouds (d). Red dots highlight significant ions. The trend line with a shorter drift time corresponds to amylase whereas the trend line with a longer drift time corresponds to dextran. Figure amended from Rashid et al.[80]

As might be expected, the mobility decreases with increasing DP or increasing oligomerization. Dextran, for the most part, exists as more extended conformers compared to the other equivalent DP polysaccharides, which is postulated to be as a result of the increased flexibility associated with the a1-6 bond. The ${ }^{\mathrm{Tw}} \mathrm{CCS}_{\mathrm{N} 2}$ did not linearly increase with increasing carbohydrate chain length, suggesting that the global conformation alters upon the successive addition of monomers. Nearly all the studied oligosaccharide ATDs consist of a single species, suggesting either mutaroation at the reducing terminus is minimal or anomers are inseparable under the conditions employed in this work. The only exception was the linear DP6 maltohexaose, which existed as two structures. However, only a single structure was observed after reduction and remarkably also after exchange of 18 of the 20 exchangeable hydrogen atoms (within the hydroxyl groups) with deuterium (Figure 18). This result was attributed to perturbation of the hydrogen bonding network within the maltohexaose. Maltooligosaccharides have been reported to adopt rigid left-handed V6 helices with six sugar units per turn in solution. Formation of the necessary intra-helical hydrogen-bonding interactions between the reducing and non-reducing termini of this V6 helix is probably dependent on the reducing residue mutarotation isoform.[182-183] The structure of the DP6 sugar is therefore likely to be at the interface between existing as a linear-like chain and the V6 helical structure. Pullulans ( $\alpha 1-4$-linked maltotriose connected by $\alpha 1$-6-linkages) were poorly separated from dextrans by IMS alone in agreement with solution-phase measurements where they are known to both adopt a similar random coil structure.[184] However, these structures are readily distinguished by their CID tandem mass spectra. These studies enabled the characterization of the enzymatic product resulting from the action of Mycobacterium tuberculosis GlgB, a potential anti-tuberculosis drug target, on 
maltooligosaccharides.[80] This enzyme is known to transfer a portion of linear maltooligosaccharide onto the 6-OH position of the maltooligosaccharide backbone forming conformationally extended, branched structures. The same year, Huang et al. have reported the IMS separation and $\mathrm{MS}^{2}$ characterization of 3-aminoquinoline labelled isomeric oligogalacturonic acids (derived from pectin cell wall) in a mixture of oligosaccharides bearing various degrees of polymerization and levels of methyl esterification including certain methyl ester regioisomers.[185-186] This suggests specific sites of methyl esterification affect the conformation more than others. However, there were instances where certain oligogalacturonic species were inseparable. In order to improve the separation of the isomeric galacturonic acid residues, the same group reported on-line hydrophilic interaction chromatography (HILIC) prior to TWIMS.[187] HILIC-TWIMS-MS ${ }^{2}$ allowed improved separation and discrimination of isomers. However, even using both HILIC and TWIMS separation, some isomers still remained extremely challenging to resolve. Estimations of the theoretical $\mathrm{CCS}_{\mathrm{N} 2}$ values highlighted that the current resolving power of TWIMS is insufficient to separate all isomeric methyl esterified oligogalacturonic acids completely. Other complex mixtures of oligosaccharides from a digest of sugar beet pectin have been subsequently studied, since they contain a large variety of both unknown structures and potential isomers. The combination of HILIC with TWIMS enabled simultaneous separation and characterization of isomeric beet rhamnogalacturonan I derived oligosaccharides based on the position of their glucuronic acid substituents. However, again, all isomers were not resolved using IMS alone due to the small differences in their ${ }^{\mathrm{TW}} \mathrm{CCS}_{\mathrm{N} 2}$ values. Nevertheless, some novel structural isomers were identified for the first time, which demonstrated that i) oligosaccharide structures from beet pectin digest were more complex than previously reported and ii) IMS is suitable for the discovery of new structures.

Finally, in the recent study of Plancot et al., IM-MS approaches were able to characterize arabinoxylan fragments obtained after endo-xylanase digestion of leaf extracts from two African plant species Eragrostis nindensis and Eragrostis tef.[188] Multiple species were observed within the ATD suggesting the existence of structural isomers, confirmed by post-mobility tandem MS. Based on IMMS/MS experiments for a DP4 oligosaccharide, the isomer at the shorter drift times could be assigned to a branched arabinoxylan structure and the one at the longer drift times to the linear one (Figure 19). However, although the presence of isomers was evidenced by IMS, fragments having higher DP were unresolvable. 


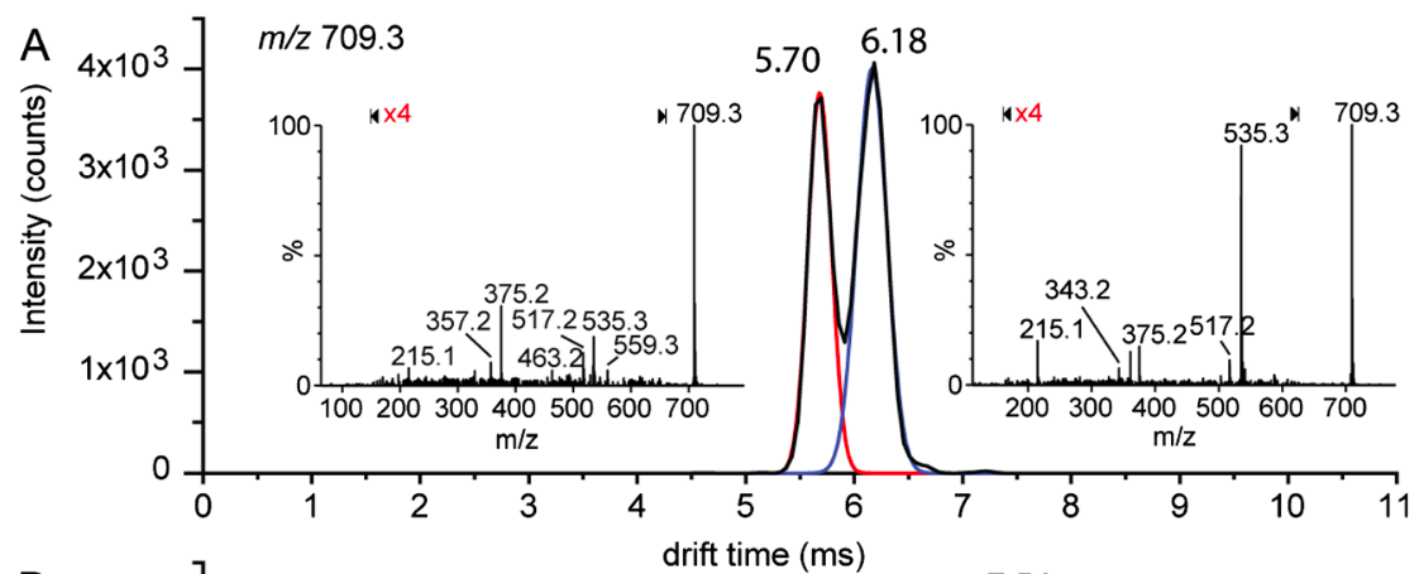

Figure 19 - Extracted ion mobility spectra of DP4 isomers $(m / z$ 709). The insets show the tandem mass spectra associated with the drift times 5.70 and 6.18 ms. Figure taken from Plancot et al.[188]

\subsection{Glycopeptides/Glycoproteins}

The complexity of analysing glycoproteins and glycopeptides as a single, intact entity is increased due the isomeric nature of many of the carbohydrate moities. These factors have meant that MS techniques alone have often struggled to separate and therefore elucidate the different carbohydrate structures present and also define sites of glycosylation. With incorporation of IMS, analysis at the intact level has begun to emerge. The additional analytical dimension removes the requirement for lengthy deglycosylation and clean-up steps and in many cases has enabled simultaneous separation and identification.

Use of collisional activation or collision induced unfolding (CIU) has been reported during IMS experiments due to its ability to induce a change in conformation, identifiable by IMS. Leary studied the effects of increasing the trap collision energy of a TWIMS instrument on the ATDs and mobility profiles of the small glycoprotein, MCP-1.[189] Monocyte chemotactic protein 1 (MCP-1) is a chemo-attractant cytokine that recruits monocytes, dendritic cells, and memory $\mathrm{T}$ cells to the sites of inflammation. MCP-1 exists in equilibrium between monomer, dimer and higher oligomeric forms; MCP-1 monomer activates its cognate receptors, while oligomerization and glycosaminoglycan (GAG) binding has been proven essential for in vivo activity. IMS was used to structurally probe the MCP-1 monomer, the non-covalent MCP-1 dimer and a dimer analogue non-covalently bound to a semi-synthetic GAG (Figure 20). With increasing collision energy, longer arrival times were observed for both dimers, indicative of a more extended conformation. However, the monomer species remained compact at equivalent activation energies. Comparison of the bound and unbound dimers revealed that GAG binding stabilized the dimer complex towards dissociation, indicating that the interaction was across the two MCP-1 monomers. In a second paper by the same group, IMS was used to show different structural specificity for GAG binding to two different chemokine variations. The interactions of chemokines with GAG moieties are essential for regulating chemokine 
oligomerization, cell migration and cellular recruitment and so an understanding of how the overall conformation is affected upon binding is sought after.[190] CIU has also been explored recently by the Ruotolo group for the larger, more complex system of a monoclonal antibody (mAb).[191] Through use of collision energy ramps, mobility acquisition and heat map plots, the authors were able to demonstrate how the levels of glycosylation affect the unfolding of the mAb.
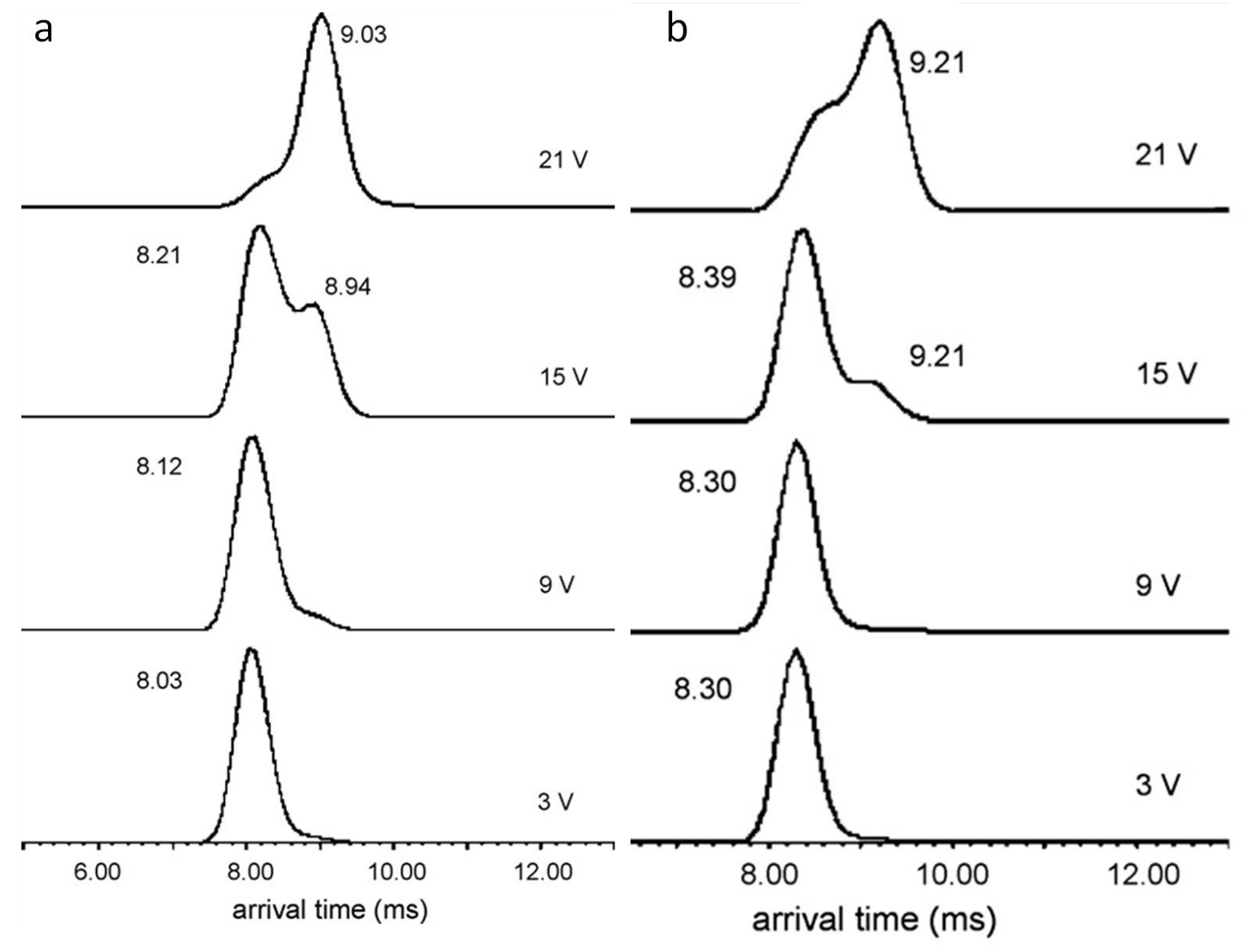

Figure 20 - The arrival time distributions (ATD) associated with the 9+ MCP-1 dimer, isolated in the quadrupole region, at the labelled collision energies 3, 9, 15, 21 and $27 \mathrm{~V}$ (a). Binding of the semi-synthetic GAG analogue Arixta is observed to stabilize the dimer at higher collision energies, although results in a less compact conformation (b). Figure amended from Schenauer et al.[189]

DTIMS has also been applied by the Barran group to calculate ${ }^{\mathrm{DT}} \mathrm{CCS}_{\mathrm{He}}$ values for betadefensins as free peptides and also for the glycoprotein equivalents; beta-defensins bound to heparinderived disaccharides (HDD).[192] The authors observed a 5\% increase in ${ }^{\mathrm{DT}} \mathrm{CCS}_{\mathrm{He}}$ for the bound form signifying a strong interaction with a defined structure, further evidenced by the narrower ATD compared with the unbound form. Molecular modelling provided $\mathrm{CCS}_{\mathrm{He}}$ values for comparison, although in all cases the ${ }^{\mathrm{DT}} \mathrm{CCS}_{\mathrm{He}}$ values for bound HDD were less than expected. The conformations observed indicate that linear defensins may still access a functional fold. Similar techniques were applied to examine the interaction of metamorphic protein lymphotactin with fondaparinux. Upon titration of the carbohydrate with the protein, rapid aggregation was observed, which resulted in $\mu \mathrm{m}$ 
assemblies.[193] Mutated and truncated forms of the protein formed complexes of different stoichiometries with distinctive ${ }^{\mathrm{DT}} \mathrm{CCS}_{\mathrm{He}}$. These studies highlight how combining predictive methodologies such as molecular modelling with structural techniques such as IM can identify functional forms of proteins and interactions that can help to discern mode of action as well the formation of macromolecular structures.

In addition to structural information, IMS enables separation of isomeric structures with the same $\mathrm{m} / z$. Li et al. used a trend line separation method coupled with IMS to dramatically increase the signal-to-noise ratio of glycopeptides derived from a glycoprotein using trypsin digestion.[194] One of the glycoproteins analysed has a significant carbohydrate content of approximately $45 \%$ across 5 $\mathrm{N}$-glycosylation sites. With such a high carbohydrate content, the likelihood of isomeric carbohydrates and hence isomeric or stereochemical glycopeptides is high. For some glycopeptides, multiple features in the ATD were observed for a single $\mathrm{m} / \mathrm{z}$ emphasizing the ability of IMS to distinguish between isomeric glycopeptides.[194] Separation of isomeric glycopeptides, differing only by the site of glycosylation, has also been reported by Creese and Cooper using FAIMS (Figure 21).[195] Combining FAIMS with routine bottom-up proteomic strategies has been shown to facilitate comprehensive mapping of the O-glycosylation profile of a glycoprotein, including identification of novel glycans and glycosylation sites.[196]

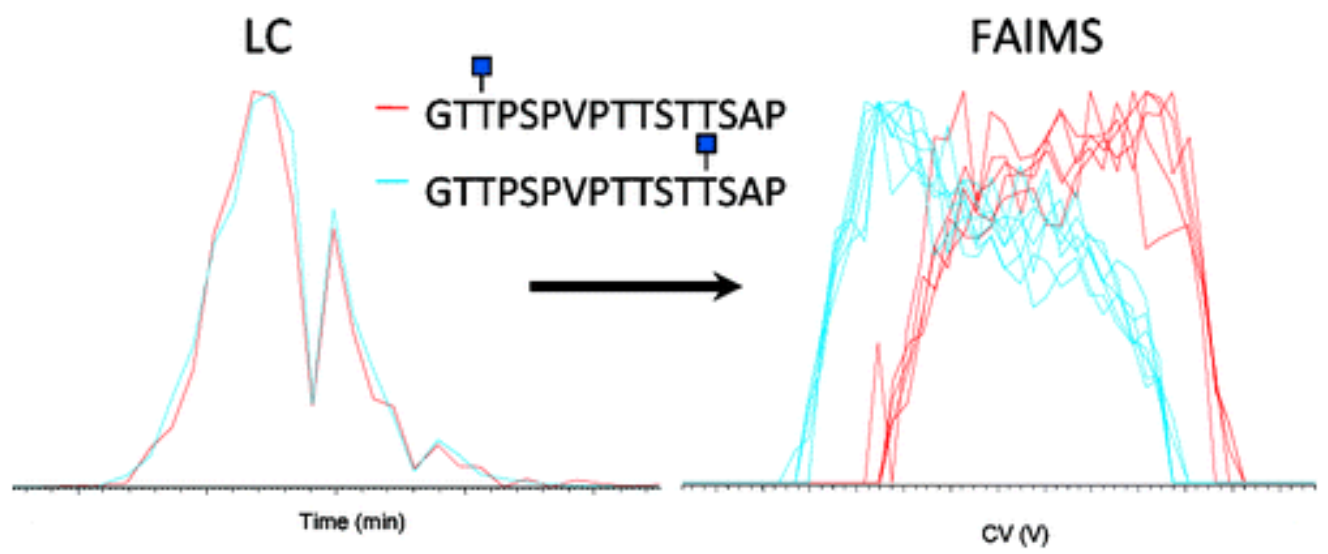

Figure 21 - LCchromatogram highlighting lack of separation of two glycopeptides differing only by their site of glycosylation and the equivalent FAIMS trace where they are distinguishable. Figure taken from Creese.[195] .

Analysis of bound glycoproteins and glycopeptides without substantial deglycosylation and/or digestion and/or purification is still relatively unexplored due to the challenges faced by MS techniques being unable to distinguish between the isomeric forms of the carbohydrates and their linked locations. However, incorporation of IMS has offered an additional dimension, allowing for the separation and identification of glycans without the need for removal from the peptide backbone. An advantageous side effect of these analyses is that structural characteristics at the intact level are observable and can offer hypotheses for functionality requirements.

\subsection{Glycolipids}


Glycolipids are amphiphilic constituents of cell membranes containing a hydrophilic carbohydrate, varying from simple monosaccharides to complex polysaccharides, covalently bound to a hydrophobic lipid moiety. The growing interest in profiling cellular glycolipids has been motivated by the importance of the carbohydrate head groups in many biological processes including recognition events, adhesion and signalling pathways as well as their architectural role in cell membranes.[197] Among the glycolipid family, lipopolysaccharides (LPS) from bacterial origin may modulate the immune response and have been extensively used as components of antibacterial vaccines against a large variety of bacteria such as Heamophilus influenzae type b,[198-199] Neisseria meningitidis type C[200] and Streptococcus pneumonia.[201-202] Animals also contain the more complex glycosphingolipids, especially within the central nervous system, where the glycan part is linked to a ceramide moiety (composed of a sphingosine and a fatty acid).[203-204] They are also reported to be vital for brain development, neuritogenesis, synaptic transmission and aging[205] and also for many diseases such as Alzheimer's, Huntington's, Parkinson's, Niemann-Pick type C, Gaucher's and Guillain-Barré syndrome.[206-211] Moreover, aberrant expressions of certain glycosphingolipids have been linked to several types of cancer, and hence, they have been frequently used as diagnostic biomarkers for different stages of cancer.[212] Because of both their biological relevant functions and promising medical application, it seems essential to well characterize this family of glycoconjugates. However, analysis of glycolipids is not a simple task due to the inherent heterogeneity and complexity of their structure including the nature, number and position of the different monosaccharide residues, as well as the length, saturation and configuration of the carbon chains of lipid moieties.

$\mathrm{Li}$ and co-workers were the first to report the combination of capillary electrophoresis (CE) and FAIMS-MS in order to analyse a mixture of O-deactylated LPS from Heamophilus influenzae strain 375.[213] Although the potential of CE-FAIMS-MS to separate a complex mixture of LPS was demonstrated, FAIMS was solely used in this study to 'filter out' the chemical background, thus enhancing detection limits. In 2007, Woods et al. studied the spatial distribution of cerebrosides species in rat brain section using MALDI-MS coupled to IMS.[214] A few years later, Shvartsburg et $a l$. described the separation of a variety of lipids using FAIMS. They have shown that monogalactosyl diacylglycerols (MGDG) and digalactosyl diacylglycerols (DGDG) fall on the same trend line within a 2D plot of compensation voltage and $\mathrm{m} / \mathrm{z}$ and thus their MS information could be readily isolated from a complex mixture of lipids. The same year, Woods and co-workers reported the use of MALDIIM-TOF to separate a mixture of gangliosides, a well-studied class of glycosphingolipids, containing different proportions of negatively charged sialic acid ( $N$-acetylneuraminic acid) as caesium adducts. Although two purified structural isomers GD1a and GD1b, which only differ by the position of the two sialic acids, generated identical MS spectra, GD1a had a greater mobility compared to GD1b. IMS was able to separate, based on their degree of sialylation (i.e. GM1, GD1 and GT1), several gangliosides extracted from a mouse cerebrum, although the structural isomers GD1a and GD1b were 
poorly resolved in most cases.[215] In a recent study, the group of Woods also reported the analysis of similar isomers using IM-MS in negative ion mode circumventing the need for doping the sample with caesium salts, which would otherwise suppress the intensity of the studied glycolipids.[216] Signal is also suppressed by the presence of phospatidylcholines and sphingomyelins, which both contain quaternary amines and so ionize very well in positive ion mode. However, even in negative ion mode the GD1 isomers were inseparable based upon their mobility.[216] Notable trends were observed for the effect of lipid structure upon mobility for the negatively charged species akin to those mentioned for salt adducts. [217] Reducing the head size, increasing the radyl chain length, increasing degree of saturation, and increasing the number of sialic acids all increase the mobility.[216]

\section{Concluding Remarks and Future Outlook}

Over the past decade, the major advances in MS-based glycomic and glycoproteomic techniques, as well as chromatographic separation methods, have allowed identification of subtle structural changes associated with disease development and progression.[218-219] However, so far, only a few reports have described the use of IM-MS to perform this task especially for glycomic applications, where additional separation technologies and structural information are urgently needed.[27-28, 134, 220] The resolving power of DTIMS,[116] TWIMS[73] and FAIMS[221] have improved significantly over a relatively short period of time, addressing initial issues of poor separation of certain isomeric carbohydrates. Also alternative forms of mobility separation are being developed, which show significant advances in resolving power. Kurulugama et al. developed a strategy termed overtone mobility spectrometry (OMS), which yields significantly improved resolving powers than the DTIMS from which it is derived. This instrument consists of a segmented drift tube, to which a uniform potential is applied across segment(s) at regular intervals forming a 'sawtooth' gradient along the mobility cell. These phases are alternatively switched on and off and ions are only transmitted when their mobilities are in resonance with the phase change frequency. An interesting result is that overtones are observed.[113, 222-227] Cyclotron geometry segmented drift cell have also been developed,[228-230] where ion packets are propelled around the tube by changing the drift field at a frequency resonant with the ions drift time through each region of the cyclotron. Ions out of resonance are not stable; therefore the cyclotron acts to trap ions of a given mobility. Increasing the number of cycles improves the resolving power ( $>300$ for more than 10 cycles and $>1000$ for 100 cycles).[229-230] The downside of this is increased ion loss as the number of cycles is increased and a reduction of duty cycle, both of which reduce the sensitivity. Either way, improvement and optimization of this approach or development of novel technologies, will see the resolving power significantly improved with sufficient sensitivity and speed for analysis of biological glycans. 
Improvements associated with the speed, structure generation and usability of computational simulations (i.e. ab initio, semi-empirical or molecular dynamics) to generate theoretical candidate structures and CCSs will also facilitate validation and characterization of dynamic structural/conformational information measured by IM-MS ${ }^{\mathrm{n}}$.

Given that the structure, presence and proportion of specific glycan-biomarkers can change during the course of disease and these structures also vary from individual-to-individual, there will undoubtedly be benefits in developing glycan-based diagnostic tools for personalized medicine. As a result, rapid, sensitive and robust methods to characterize and identify biological glycans are required in order to carry out both diagnosis and prognosis, especially approaches that can detect diseases early. In fact, structural identification of aberrant glycosylation or specific structures associated with disease have already proved beneficial in the development of a large number of carbohydrate-based vaccines over the last 20 years such as anticancer,[231] antibacterial[232] or antiviral[233] with many more still in development.[234] There will also be the requirement to assess the quality of any isolated or synthesized carbohydrate-based medicines, further driving the requirement of robust analytical techniques. Initial applications of IM-MS to facilitate separation and identification of glycans,[79, 107, 114, 122, 148, 235] glycosides,[47, 134, 215-216] polysaccharides,[82, 173, 177] glyco(peptides/proteins)[195] and glycan-based biomarkers[26-28, 134] are extremely promising, and further development and the recent commercialization of IMS technologies will inevitably increase its use within research and healthcare. The development of universal sequencing tools for carbohydrates with comparable speed and throughput to those used for nucleic acids and proteins remains a challenge, and IM-MS techniques are promising to be useful tools in this context.

\section{Acknowledgements}

We are grateful to the BBSRC (studentship to CJG, RU, LM and funding for the IBCarb network), Covance Laboratories (icase to RU) the FP7 programme (GlycoBioM, Immunoshape, GLYCOVAX) and the Royal Society (Wolfson Merit award to SLF) for supporting our work.

\section{References}

[1] K. Ohtsubo, J.D. Marth, Glycosylation in Cellular Mechanisms of Health and Disease, Cell, 126 (2006) 855-867.

[2] T. Yoshida-Moriguchi, L.P. Yu, S.H. Stalnaker, S. Davis, S. Kunz, M. Madson, M.B.A. Oldstone, H. Schachter, L. Wells, K.P. Campbell, O-Mannosyl Phosphorylation of Alpha-Dystroglycan Is Required for Laminin Binding, Science, 327 (2010) 88-92.

[3] M. Phillips, E. Nudelman, F. Gaeta, M. Perez, A. Singhal, S. Hakomori, J. Paulson, ELAM-1 mediates cell adhesion by recognition of a carbohydrate ligand, sialyl-Lex, Science, 250 (1990) 11301132.

[4] L. Bode, Human Milk Oligosaccharides: Every Baby needs a Sugar Mama, Glycobiology, (2012). 
[5] M.B. Poulin, Y. Shi, C. Protsko, S.A. Dalrymple, D.A.R. Sanders, B.M. Pinto, T.L. Lowary, Specificity of a UDP-GalNAc Pyranose-Furanose Mutase: A Potential Therapeutic Target for Campylobacter jejuni Infections, ChemBioChem, 15 (2014) 47-56.

[6] T. Boren, P. Falk, K.A. Roth, G. Larson, S. Normark, Attachment of Helicobacter-pylori to human gastric epithelium mediated by blood-group antigens, Science, 262 (1993) 1892-1895.

[7] M. Sperandio, C.A. Gleissner, K. Ley, Glycosylation in immune cell trafficking, Immunological Reviews, 230 (2009) 97-113.

[8] H.R. Morris, A. Dell, R.L. Easton, M. Panico, H. Koistinen, R. Koistinen, S. Oehninger, M.S. Patankar, M. Seppala, G.F. Clark, Gender-specific Glycosylation of Human Glycodelin Affects Its Contraceptive Activity, J. Biol. Chem., 271 (1996) 32159-32167.

[9] S. Benoff, Carbohydrates and fertilization: an overview, Molecular Human Reproduction, 3 (1997) 599-637.

[10] S. Schröter, C. Osterhoff, W. McArdle, R. Ivell, The glycocalyx of the sperm surface, Human Reproduction Update, 5 (1999) 302-313.

[11] J. Bones, S. Mittermayr, N. O'Donoghue, A. Guttman, P.M. Rudd, Ultra Performance Liquid Chromatographic Profiling of Serum N-Glycans for Fast and Efficient Identification of Cancer Associated Alterations in Glycosylation, Anal. Chem., 82 (2010) 10208-10215.

[12] G. Lauc, J.E. Huffman, M. Pucic, L. Zgaga, B. Adamczyk, A. Muzinic, M. Novokmet, O. Polasek, O. Gornik, J. Kristic, T. Keser, V. Vitart, B. Scheijen, H.W. Uh, M. Molokhia, A.L. Patrick, P. McKeigue, I. Kolcic, I.K. Lukic, O. Swann, F.N. van Leeuwen, L.R. Ruhaak, J.J. HouwingDuistermaat, P.E. Slagboom, M. Beekman, A.J.M. de Craen, A.M. Deelder, Q. Zeng, W. Wang, N.D. Hastie, U. Gyllensten, J.F. Wilson, M. Wuhrer, A.F. Wright, P.M. Rudd, C. Hayward, Y. Aulchenko, H. Campbell, I. Rudan, Loci Associated with N-Glycosylation of Human Immunoglobulin G Show Pleiotropy with Autoimmune Diseases and Haematological Cancers, PLoS Genet., 9 (2013).

[13] T. Iskratsch, A. Braun, K. Paschinger, I.B.H. Wilson, Specificity analysis of lectins and antibodies using remodeled glycoproteins, Anal. Biochem., 386 (2009) 133-146.

[14] S.R. Kumar, E.R. Sauter, T.P. Quinn, S.L. Deutscher, Thomsen-Friedenreich and Tn Antigens in Nipple Fluid: Carbohydrate Biomarkers for Breast Cancer Detection, Clinical Cancer Research, 11 (2005) 6868-6871.

[15] R.M. Anthony, F. Nimmerjahn, D.J. Ashline, V.N. Reinhold, J.C. Paulson, J.V. Ravetch, Recapitulation of IVIG Anti-Inflammatory Activity with a Recombinant IgG Fc, Science, 320 (2008) 373-376.

[16] Y. Kanda, T. Yamada, K. Mori, A. Okazaki, M. Inoue, K. Kitajima-Miyama, R. Kuni-Kamochi, R. Nakano, K. Yano, S. Kakita, K. Shitara, M. Satoh, Comparison of biological activity among nonfucosylated therapeutic IgG1 antibodies with three different N-linked Fc oligosaccharides: the high-mannose, hybrid, and complex types, Glycobiology, 17 (2007) 104-118.

[17] G. Thanabalasingham, J.E. Huffman, J.J. Kattla, M. Novokmet, I. Rudan, A.L. Gloyn, C. Hayward, B. Adamczyk, R.M. Reynolds, A. Muzinic, N. Hassanali, M. Pucic, A.J. Bennett, A. Essafi, O. Polasek, S.A. Mughal, I. Redzic, D. Primorac, L. Zgaga, I. Kokic, T. Hansen, D. Gasperikova, E. Tjora, M.W.J. Strachan, T. Nielsen, J. Stanik, I. Klimes, O.B. Pedersen, P.R. Njolstad, S.H. Wild, U. Gyllensten, O. Gornik, J.F. Wilson, N.D. Hastie, H. Campbell, M.I. McCarthy, P.M. Rudd, K.R. Owen, G. Lauc, A.F. Wright, Mutations in HNF1A Result in Marked Alterations of Plasma Glycan Profile, Diabetes, 62 (2013) 1329-1337.

[18] H.F. Lodish, A. Berk, S. Zipursky, P. Matsudaira, D. Baltimore, J. Darnell, Molecular Cell Biology, 4th edition ed., New York, 2000.

[19] M. Wuhrer, C.A.M. Koeleman, J.M. Fitzpatrick, K.F. Hoffmann, A.M. Deelder, C.H. Hokke, Gender-specific expression of complex-type N-glycans in schistosomes, Glycobiology, 16 (2006) 991-1006.

[20] F. Yamamoto, ABO blood group system--ABH oligosaccharide antigens, anti-A and anti-B, A and B glycosyltransferases, and ABO genes, Immunohematology / American Red Cross, 20 (2004) 322.

[21] J. Krištić, F. Vučković, C. Menni, L. Klarić, T. Keser, I. Beceheli, M. Pučić-Baković, M. Novokmet, M. Mangino, K. Thaqi, P. Rudan, N. Novokmet, J. Šarac, S. Missoni, I. Kolčić, O. Polašek, I. Rudan, H. Campbell, C. Hayward, Y. Aulchenko, A. Valdes, J.F. Wilson, O. Gornik, D. Primorac, V. Zoldoš, T. Spector, G. Lauc, Glycans Are a Novel Biomarker of Chronological and 
Biological Ages, The Journals of Gerontology Series A: Biological Sciences and Medical Sciences, 69 (2014) 779-789.

[22] I. Gudelj, T. Keser, F. Vuckovic, V. Skaro, S.S. Goreta, T. Pavic, J. Dumic, D. Primorac, G. Lauc, O. Gornik, Estimation of human age using N-glycan profiles from bloodstains, Int. J. Legal Med., 129 (2015) 955-961.

[23] L. Krishnamoorthy, L.K. Mahal, Glycomic Analysis: An Array of Technologies, ACS Chem. Biol., 4 (2009) 715-732.

[24] O. Gornik, I. Gornik, V. Gasparovic, G. Lauc, Change in transferrin sialylation is a potential prognostic marker for severity of acute pancreatitis, Clinical Biochemistry, 41 (2008) 504-510.

[25] I.T. Akmacic, N. Ventham, E. Theodoratou, F. Vuckovic, N. Kennedy, J. Kristic, E.R. Nimmo, R. Kalla, H. Drummond, J. Stambuk, M.G. Dunlop, M. Novokmet, Y. Aulchenko, O. Gornik, H. Campbell, M. Pucic-Bakovic, J. Satsangi, G. Lauc, I.B. Consortium, Inflammatory bowel disease associates with pro-inflammatory potential of the IgG glycome, J. Crohns Colitis, 9 (2015) S107S107.

[26] D. Isailovic, R.T. Kurulugama, M.D. Plasencia, S.T. Stokes, Z. Kyselova, R. Goldman, Y. Mechref, M.V. Novotny, D.E. Clemmer, Profiling of human serum glycans associated with liver cancer and cirrhosis by IMS-MS, Journal of Proteome Research, 7 (2008) 1109-1117.

[27] D. Isailovic, M.D. Plasencia, M.M. Gaye, S.T. Stokes, R.T. Kurulugama, V. Pungpapong, M. Zhang, Z. Kyselova, R. Goldman, Y. Mechref, M.V. Novotny, D.E. Clemmer, Delineating Diseases by IMS-MS Profiling of Serum N-linked Glycans, Journal of Proteome Research, 11 (2012) 576-585. [28] M.M. Gaye, S.J. Valentine, Y. Hu, N. Mirjankar, Z.T. Hammoud, Y. Mechref, B.K. Lavine, D.E. Clemmer, Ion Mobility-Mass Spectrometry Analysis of Serum N-linked Glycans from Esophageal Adenocarcinoma Phenotypes, Journal of Proteome Research, 11 (2012) 6102-6110.

[29] K. Ohtsubo, S. Takamatsu, M.T. Minowa, A. Yoshida, M. Takeuchi, J.D. Marth, Dietary and genetic control of glucose transporter 2 glycosylation promotes insulin secretion in suppressing diabetes, Cell, 123 (2005) 1307-1321.

[30] C.R. Varki A, Esko JD, et al., Essentials of Glycobiology, 2 ed., Cold Spring Harbor, New York, 2009.

[31] L. Royle, A. Roos, D.J. Harvey, M.R. Wormald, D. Van Gijlswijk-Janssen, E.-R.M. Redwan, I.A. Wilson, M.R. Daha, R.A. Dwek, P.M. Rudd, Secretory IgA N- and O-Glycans Provide a Link between the Innate and Adaptive Immune Systems, J. Biol. Chem., 278 (2003) 20140-20153.

[32] E. Gorelik, U. Galili, A. Raz, On the role of cell surface carbohydrates and their binding proteins (lectins) in tumor metastasis, Cancer Metastasis Rev, 20 (2001) 245-277.

[33] M.J. Paszek, C.C. DuFort, O. Rossier, R. Bainer, J.K. Mouw, K. Godula, J.E. Hudak, J.N. Lakins, A.C. Wijekoon, L. Cassereau, M.G. Rubashkin, M.J. Magbanua, K.S. Thorn, M.W. Davidson, H.S. Rugo, J.W. Park, D.A. Hammer, G. Giannone, C.R. Bertozzi, V.M. Weaver, The cancer glycocalyx mechanically primes integrin-mediated growth and survival, Nature, 511 (2014) 319-325. [34] F. Jacob, D.R. Goldstein, N.V. Bovin, T. Pochechueva, M. Spengler, R. Caduff, D. Fink, M.I. Vuskovic, M.E. Huflejt, V. Heinzelmann-Schwarz, Serum antiglycan antibody detection of nonmucinous ovarian cancers by using a printed glycan array, Int. J. Cancer, 130 (2012) 138-146.

[35] G.F. Springer, T and Tn, general carcinoma auto-antigens, Science, 224 (1984) 1198-1206.

[36] Y.T. Chen, Y.M. Chong, C.W. Cheng, C.L. Ho, H.W. Tsai, F.H. Kasten, Y.L. Chen, C.F. Chang, Identification of novel tumor markers for oral squamous cell carcinoma using glycoproteomic analysis, Clin. Chim. Acta, 420 (2013) 45-53.

[37] D.M. Krasnewich, G.D. Holt, M. Brantly, F. Skovby, J. Redwine, W.A. Gahl, Abnormal synthesis of dolichol-linked oligosaccharides in carbohydrate-deficient glycoprotein syndrome, Glycobiology, 5 (1995) 503-510.

[38] F. Vuckovic, J. Kristic, I. Gudelj, M. Teruel, T. Keser, M. Pezer, M. Pucic-Bakovic, J. Stambuk, I. Trbojevic-Akmacic, C. Barrios, T. Pavic, C. Menni, Y.X. Wang, Y. Zhou, L.F. Cui, H.C. Song, Q. Zeng, X.H. Guo, B.A. Pons-Estel, P. McKeigue, A.L. Patrick, O. Gornik, T.D. Spector, M. Harjacek, M. Alarcon-Riquelme, M. Molokhia, W. Wang, G. Lauc, Association of Systemic Lupus Erythematosus With Decreased Immunosuppressive Potential of the IgG Glycome, Arthritis Rheumatol., 67 (2015) 2978-2989. 
[39] A. Weidemann, G. König, D. Bunke, P. Fischer, J.M. Salbaum, C.L. Masters, K. Beyreuther, Identification, biogenesis, and localization of precursors of Alzheimer's disease A4 amyloid protein, Cell, 57 (1989) 115-126.

[40] P. Pahlsson, S.L. Spitalnik, The role of glycosylation in synthesis and secretion of beta-amyloid precursor protein by Chinese hamster ovary cells, Archives of Biochemistry and Biophysics, 331 (1996) 177-186.

[41] J. Clardy, C. Walsh, Lessons from natural molecules, Nature, 432 (2004) 829-837.

[42] M. Dalziel, M. Crispin, C.N. Scanlan, N. Zitzmann, R.A. Dwek, Emerging Principles for the Therapeutic Exploitation of Glycosylation, Science, 343 (2014).

[43] M.N. Thaker, G.D. Wright, Opportunities for Synthetic Biology in Antibiotics: Expanding Glycopeptide Chemical Diversity, ACS Synth. Biol., 4 (2015) 195-206.

[44] L. Royle, M.P. Campbell, C.M. Radcliffe, D.M. White, D.J. Harvey, J.L. Abrahams, Y.G. Kim, G.W. Henry, N.A. Shadick, M.E. Weinblatt, D.M. Lee, P.M. Rudd, R.A. Dwek, HPLC-based analysis of serum N-glycans on a 96-well plate platform with dedicated database software, Anal. Biochem., $376(2008) 1-12$.

[45] M.P. Campbell, L. Royle, C.M. Radcliffe, R.A. Dwek, P.M. Rudd, GlycoBase and autoGU: tools for HPLC-based glycan analysis, Bioinformatics, 24 (2008) 1214-1216.

[46] S. Itoh, N. Kawasaki, M. Ohta, M. Hyuga, S. Hyuga, T. Hayakawa, Simultaneous microanalysis of N-linked oligosaccharides in a glycoprotein using microbore graphitized carbon column liquid chromatography-mass spectrometry, Journal of Chromatography A, 968 (2002) 89-100.

[47] M. Pičmanová, E.H. Neilson, M.S. Motawia, C.E. Olsen, N. Agerbirk, C.J. Gray, S. Flitsch, S. Meier, D. Silvestro, K. Jørgensen, R. Sánchez Pérez, B.L. Møller, N. Bjarnholt, A recycling pathway for cyanogenic glycosides evidenced by the comparative metabolic profiling in three cyanogenic plant species, Biochem. J., 469 (2015) 375-389.

[48] L.R. Ruhaak, A.M. Deelder, M. Wuhrer, Oligosaccharide analysis by graphitized carbon liquid chromatography-mass spectrometry, Anal. Bioanal. Chem., 394 (2009) 163-174.

[49] C.A. Cooper, E. Gasteiger, N.H. Packer, GlycoMod - A software tool for determining glycosylation compositions from mass spectrometric data, Proteomics, 1 (2001) 340-349.

[50] H.L. Zhang, S. Singh, V.N. Reinhold, Congruent strategies for carbohydrate sequencing. 2. FragLib: An MSn spectral library, Anal. Chem., 77 (2005) 6263-6270.

[51] C.A. Cooper, M.J. Harrison, M.R. Wilkins, N.H. Packer, GlycoSuiteDB: a new curated relational database of glycoprotein glycan structures and their biological sources, Nucleic Acids Res., 29 (2001) 332-335.

[52] O.M. Saad, J.A. Leary, Heparin sequencing using enzymatic digestion and ESI-MSn with HOST: A heparin/HS oligosaccharide sequencing tool, Anal. Chem., 77 (2005) 5902-5911.

[53] E.P. Go, K.R. Rebecchi, D.S. Dalpathado, M.L. Bandu, Y. Zhang, H. Desaire, GlycoPep DB: A tool for glycopeptide analysis using a "smart search", Anal. Chem., 79 (2007) 1708-1713.

[54] C.A. Hayes, N.G. Karlsson, W.B. Struwe, F. Lisacek, P.M. Rudd, N.H. Packer, M.P. Campbell, UniCarb-DB: a database resource for glycomic discovery, Bioinformatics, 27 (2011) 1343-1344.

[55] M. Frank, T. Lutteke, C.W. von der Lieth, GlycoMapsDB: a database of the accessible conformational space of glycosidic linkages, Nucleic Acids Res., 35 (2007) D287-D290.

[56] M.P. Campbell, T. Nguyen-Khuong, C.A. Hayes, S.A. Flowers, K. Alagesan, D. Kolarich, N.H. Packer, N.G. Karlsson, Validation of the curation pipeline of UniCarb-DB: Building a global glycan reference MS/MS repository, BBA-Proteins Proteomics, 1844 (2014) 108-116.

[57] C. Konda, F.A. Londry, B. Bendiak, Y. Xia, Assignment of the Stereochemistry and Anomeric Configuration of Sugars within Oligosaccharides Via Overlapping Disaccharide Ladders Using MSn, J. Am. Soc. Mass Spectrom., 25 (2014) 1441-1450.

[58] B. Domon, C.E. Costello, A systematic nomenclature for carbohydrate fragmentations in FABMS/MS spectra of glycoconjugates, Glycoconjugate J., 5 (1988) 397-409.

[59] C.S. Creaser, J.R. Griffiths, C.J. Bramwell, S. Noreen, C.A. Hill, C.L.P. Thomas, Ion mobility spectrometry: a review. Part 1. Structural analysis by mobility measurement, Analyst, 129 (2004) 984994.

[60] F. Lanucara, S.W. Holman, C.J. Gray, C.E. Eyers, The power of ion mobility-mass spectrometry for structural characterization and the study of conformational dynamics, Nat Chem, 6 (2014) 281294. 
[61] C. Uetrecht, R.J. Rose, E. van Duijn, K. Lorenzen, A.J.R. Heck, Ion mobility mass spectrometry of proteins and protein assemblies, Chem. Soc. Rev., 39 (2010) 1633-1655.

[62] A.B. Kanu, P. Dwivedi, M. Tam, L. Matz, H.H. Hill, Ion mobility-mass spectrometry, Journal of Mass Spectrometry, 43 (2008) 1-22.

[63] T. Wyttenbach, N.A. Pierson, D.E. Clemmer, M.T. Bowers, Ion Mobility Analysis of Molecular Dynamics, in: M.A. Johnson, T.J. Martinez (Eds.) Annual Review of Physical Chemistry, Vol 65, vol. 65, 2014, pp. 175-196.

[64] V. D'Atri, M. Porrini, F. Rosu, V. Gabelica, Linking molecular models with ion mobility experiments. Illustration with a rigid nucleic acid structure, Journal of Mass Spectrometry, 50 (2015) 711-726.

[65] E.A. Mason, H.W. Schamp, Mobility of gaseous ions in weak electric fields, Ann. Phys., 4 (1958) 233-270.

[66] R.T. Kurulugama, E. Darland, F. Kuhlmann, G. Stafford, J. Fjeldsted, Evaluation of drift gas selection in complex sample analyses using a high performance drift tube ion mobility-QTOF mass spectrometer, Analyst, 140 (2015) 6834-6844.

[67] H.W. Ellis, R.Y. Pai, E.W. McDaniel, E.A. Mason, L.A. Viehland, Transport properties of gaseous ions over a wide energy range, Atomic Data and Nuclear Data Tables, 17 (1976) 177-210.

[68] H.W. Ellis, E.W. McDaniel, D.L. Albritton, L.A. Viehland, S.L. Lin, E.A. Mason, Transport properties of gaseous ions over a wide energy range. Part II, Atomic Data and Nuclear Data Tables, 22 (1978) 179-217.

[69] H.W. Ellis, M.G. Thackston, E.W. McDaniel, E.A. Mason, Transport-properties of gaseous-ions over a wide energy-range .3, Atomic Data and Nuclear Data Tables, 31 (1984) 113-151.

[70] L.A. Viehland, E.A. Mason, Transport-properties of gaseous-ions over a wide energy-range .4, Atomic Data and Nuclear Data Tables, 60 (1995) 37-95.

[71] J.C. May, C.R. Goodwin, N.M. Lareau, K.L. Leaptrot, C.B. Morris, R.T. Kurulugama, A. Mordehai, C. Klein, W. Barry, E. Darland, G. Overney, K. Imatani, G.C. Stafford, J.C. Fjeldsted, J.A. McLean, Conformational Ordering of Biomolecules in the Gas Phase: Nitrogen Collision Cross Sections Measured on a Prototype High Resolution Drift Tube Ion Mobility-Mass Spectrometer, Anal. Chem., 86 (2014) 2107-2116.

[72] K. Giles, S.D. Pringle, K.R. Worthington, D. Little, J.L. Wildgoose, R.H. Bateman, Applications of a travelling wave-based radio-frequency-only stacked ring ion guide, Rapid Commun. Mass Spectrom., 18 (2004) 2401-2414.

[73] K. Giles, J.P. Williams, I. Campuzano, Enhancements in travelling wave ion mobility resolution, Rapid Commun. Mass Spectrom., 25 (2011) 1559-1566.

[74] A.S. Gelb, R.E. Jarratt, Y.T. Huang, E.D. Dodds, A Study of Calibrant Selection in Measurement of Carbohydrate and Peptide Ion-Neutral Collision Cross Sections by Traveling Wave Ion Mobility Spectrometry, Anal. Chem., 86 (2014) 11396-11402.

[75] J. Hofmann, W.B. Struwe, C.A. Scarff, J.H. Scrivens, D.J. Harvey, K. Pagel, Estimating Collision Cross Sections of Negatively Charged N-Glycans using Traveling Wave Ion Mobility-Mass Spectrometry, Anal. Chem., 86 (2014) 10789-10795.

[76] C. Bleiholder, N.R. Johnson, S. Contreras, T. Wyttenbach, M.T. Bowers, Molecular Structures and Ion Mobility Cross Sections: Analysis of the Effects of He and N-2 Buffer Gas, Anal. Chem., 87 (2015) 7196-7203.

[77] M.F. Jarrold, Helices and sheets in vacuo, Phys. Chem. Chem. Phys., 9 (2007) 1659-1671.

[78] E.W. Robinson, E.R. Williams, Multidimensional separations of ubiquitin conformers in the gas phase: Relating ion cross sections to H/D exchange measurements, J. Am. Soc. Mass Spectrom., 16 (2005) 1427-1437.

[79] J.P. Williams, M. Grabenauer, R.J. Holland, C.J. Carpenter, M.R. Wormald, K. Giles, D.J. Harvey, R.H. Bateman, J.H. Scrivens, M.T. Bowers, Characterization of simple isomeric oligosaccharides and the rapid separation of glycan mixtures by ion mobility mass spectrometry, Int. J. Mass Spectrom., 298 (2010) 119-127.

[80] A.M. Rashid, G. Saalbach, S. Bornemann, Discrimination of large maltooligosaccharides from isobaric dextran and pullulan using ion mobility mass spectrometry, Rapid Commun. Mass Spectrom., 28 (2014) 191-199. 
[81] L. Jin, P.E. Barran, J.A. Deakin, M. Lyon, D. Uhrin, Conformation of glycosaminoglycans by ion mobility mass spectrometry and molecular modelling, Phys. Chem. Chem. Phys., 7 (2005) 3464-3471. [82] Y.J. Seo, M.R. Schenauer, J.A. Leary, Biologically relevant metal-cation binding induces conformational changes in heparin oligosaccharides as measured by ion mobility mass spectrometry, Int. J. Mass Spectrom., 303 (2011) 191-198.

[83] B.T. Ruotolo, K. Giles, I. Campuzano, A.M. Sandercock, R.H. Bateman, C.V. Robinson, Evidence for macromolecular protein rings in the absence of bulk water, Science, 310 (2005) 16581661.

[84] B.T. Ruotolo, J.L.P. Benesch, A.M. Sandercock, S.J. Hyung, C.V. Robinson, Ion mobility-mass spectrometry analysis of large protein complexes, Nat. Protoc., 3 (2008) 1139-1152.

[85] R. Cumeras, E. Figueras, C.E. Davis, J.I. Baumbach, I. Gracia, Review on Ion Mobility Spectrometry. Part 1: current instrumentation, Analyst, 140 (2015) 1376-1390.

[86] R.W. Purves, R. Guevremont, Electrospray ionization high-field asymmetric waveform ion mobility spectrometry-mass spectrometry, Anal. Chem., 71 (1999) 2346-2357.

[87] A.A. Shvartsburg, T.A. Seim, W.F. Danielson, R. Norheim, R.J. Moore, G.A. Anderson, R.D. Smith, High-Definition Differential Ion Mobility Spectrometry with Resolving Power up to 500, J. Am. Soc. Mass Spectrom., 24 (2013) 109-114.

[88] R. Guevremont, High-field asymmetric waveform ion mobility spectrometry: A new tool for mass spectrometry, Journal of Chromatography A, 1058 (2004) 3-19.

[89] A.A. Shvartsburg, Y.M. Ibrahim, R.D. Smith, Differential Ion Mobility Separations in up to 100 $\%$ Helium Using Microchips, J. Am. Soc. Mass Spectrom., 25 (2014) 480-489.

[90] F. Fernandez-Lima, D. Kaplan, J. Suetering, M. Park, Gas-phase separation using a trapped ion mobility spectrometer, Int. J. Ion Mobil. Spec., 14 (2011) 93-98.

[91] K. Michelmann, J. Silveira, M. Ridgeway, M. Park, Fundamentals of Trapped Ion Mobility Spectrometry, J. Am. Soc. Mass Spectrom., 26 (2015) 14-24.

[92] J.A. Silveira, M.E. Ridgeway, M.A. Park, High Resolution Trapped Ion Mobility Spectrometery of Peptides, Anal. Chem., 86 (2014) 5624-5627.

[93] S. Koeniger, D. Clemmer, Resolution and structural transitions of elongated states of ubiquitin, J. Am. Soc. Mass Spectrom., 18 (2007) 322-331.

[94] E. Mack, Average cross-sectional areas of molecules by gaseous diffusion methods, J. Am. Chem. Soc., 47 (1925) 2468-2482.

[95] G. Vonhelden, M.T. Hsu, N. Gotts, M.T. Bowers, Carbon cluster cations with up to 84 atoms strucutres, formation mechanism, and reactivity, Journal of Physical Chemistry, 97 (1993) 8182-8192. [96] M.F. Mesleh, J.M. Hunter, A.A. Shvartsburg, G.C. Schatz, M.F. Jarrold, Structural information from ion mobility measurements: Effects of the long-range potential, Journal of Physical Chemistry, 100 (1996) 16082-16086.

[97] C. Bleiholder, T. Wyttenbach, M.T. Bowers, A novel projection approximation algorithm for the fast and accurate computation of molecular collision cross sections (I). Method, Int. J. Mass Spectrom., 308 (2011) 1-10.

[98] S.E. Anderson, C. Bleiholder, E.R. Brocker, P.J. Stang, M.T. Bowers, A novel projection approximation algorithm for the fast and accurate computation of molecular collision cross sections (III): Application to supramolecular coordination-driven assemblies with complex shapes, Int. J. Mass Spectrom., 330 (2012) 78-84.

[99] C. Bleiholder, S. Contreras, M.T. Bowers, A novel projection approximation algorithm for the fast and accurate computation of molecular collision cross sections (IV). Application to polypeptides, Int. J. Mass Spectrom., 354 (2013) 275-280.

[100] C. Bleiholder, S. Contreras, T.D. Do, M.T. Bowers, A novel projection approximation algorithm for the fast and accurate computation of molecular collision cross sections (II). Model parameterization and definition of empirical shape factors for proteins, Int. J. Mass Spectrom., 345 (2013) 89-96.

[101] T. Wyttenbach, C. Bleiholder, S.E. Anderson, M.T. Bowers, A new algorithm to characterise the degree of concaveness of a molecular surface relevant in ion mobility spectrometry, Molecular Physics, 113 (2015) 2344-2349.

[102] A.A. Shvartsburg, M.F. Jarrold, An exact hard-spheres scattering model for the mobilities of polyatomic ions, Chem. Phys. Lett., 261 (1996) 86-91. 
[103] A.A. Shvartsburg, S.V. Mashkevich, E.S. Baker, R.D. Smith, Optimization of algorithms for ion mobility calculations, J. Phys. Chem. A, 111 (2007) 2002-2010.

[104] C. Larriba-Andaluz, C.J. Hogan, Jr., Collision cross section calculations for polyatomic ions considering rotating diatomic/linear gas molecules, J. Chem. Phys., 141 (2014).

[105] I. Campuzano, M.F. Bush, C.V. Robinson, C. Beaumont, K. Richardson, H. Kim, H.I. Kim, Structural Characterization of Drug-like Compounds by Ion Mobility Mass Spectrometry: Comparison of Theoretical and Experimentally Derived Nitrogen Collision Cross Sections, Anal. Chem., 84 (2012) 1026-1033.

[106] L.S. Fenn, J.A. McLean, Enhanced carbohydrate structural selectivity in ion mobility-mass spectrometry analyses by boronic acid derivatization, Chem. Commun., (2008) 5505-5507.

[107] J. Hofmann, H.S. Hahm, P.H. Seeberger, K. Pagel, Identification of carbohydrate anomers using ion mobility-mass spectrometry, Nature, 526 (2015) 241-244.

[108] P. Dwivedi, B. Bendiak, B. Clowers, H. Hill, Rapid resolution of carbohydrate isomers by electrospray ionization ambient pressure ion mobility spectrometry-time-of-flight mass spectrometry (ESI-APIMS-TOFMS), J. Am. Soc. Mass Spectrom., 18 (2007) 1163-1175.

[109] W. Gabryelski, K.L. Froese, Rapid and sensitive differentiation of anomers, linkage, and position isomers of disaccharides using High-Field Asymmetric Waveform Ion Mobility Spectrometry (FAIMS), J. Am. Soc. Mass Spectrom., 14 (2003) 265-277.

[110] D.J. Harvey, C.A. Scarff, M. Crispin, C.N. Scanlan, C. Bonomelli, J.H. Scrivens, MALDIMS/MS with Traveling Wave Ion Mobility for the Structural Analysis of N-Linked Glycans, J. Am. Soc. Mass Spectrom., 23 (2012) 1955-1966.

[111] P.M. Lalli, Y.E. Corilo, M. Fasciotti, M.F. Riccio, G.F. de Sa, R.J. Daroda, G.H.M.F. Souza, M. McCullagh, M.D. Bartberger, M.N. Eberlin, I.D.G. Campuzano, Baseline resolution of isomers by traveling wave ion mobility mass spectrometry: investigating the effects of polarizable drift gases and ionic charge distribution, Journal of Mass Spectrometry, 48 (2013) 989-997.

[112] G.A. Bataglion, G. Souza, G. Heerdt, N.H. Morgon, J.D.L. Dutra, R.O. Freire, M.N. Eberlin, A. Tata, Separation of glycosidic catiomers by TWIM-MS using CO2 as a drift gas, Journal of Mass Spectrometry, 50 (2015) 336-343.

[113] R.T. Kurulugama, F.M. Nachtigall, S. Lee, S.J. Valentine, D.E. Clemmer, Overtone Mobility Spectrometry: Part 1. Experimental Observations, J. Am. Soc. Mass Spectrom., 20 (2009) 729-737.

[114] P. Both, A.P. Green, C.J. Gray, R. Šardzík, J. Voglmeir, C. Fontana, M. Austeri, M. Rejzek, D. Richardson, R.A. Field, G. Widmalm, S.L. Flitsch, C.E. Eyers, Discrimination of epimeric glycans and glycopeptides using IM-MS and its potential for carbohydrate sequencing, Nat Chem, 6 (2014) 65-74.

[115] M. Fasciotti, G.B. Sanvido, V.G. Santos, P.M. Lalli, M. McCullagh, G.F. de Sá, R.J. Daroda, M.G. Peter, M.N. Eberlin, Separation of isomeric disaccharides by traveling wave ion mobility mass spectrometry using CO2 as drift gas, Journal of Mass Spectrometry, 47 (2012) 1643-1647.

[116] E.J. Davis, K.F. Grows, W.F. Siems, H.H. Hill, Improved Ion Mobility Resolving Power with Increased Buffer Gas Pressure, Anal. Chem., 84 (2012) 4858-4865.

[117] J.C. May, J.N. Dodds, R.T. Kurulugama, G.C. Stafford, J.C. Fjeldsted, J.A. McLean, Broadscale resolving power performance of a high precision uniform field ion mobility-mass spectrometer, Analyst, 140 (2015) 6824-6833.

[118] L.S. Fenn, J.A. McLean, Structural resolution of carbohydrate positional and structural isomers based on gas-phase ion mobility-mass spectrometry, Phys. Chem. Chem. Phys., 13 (2011) 2196-2205.

[119] S. Lee, T. Wyttenbach, M.T. Bowers, Gas phase structures of sodiated oligosaccharides by ion mobility/ion chromatography methods, Int. J. Mass Spectrom. Ion Process., 167-168 (1997) 605-614. [120] M.D. Leavell, S.P. Gaucher, J.A. Leary, J.A. Taraszka, D.E. Clemmer, Conformational studies of Zn-ligand-hexose diastereomers using ion mobility measurements and density functional theory calculations, J. Am. Soc. Mass Spectrom., 13 (2002) 284-293.

[121] Y. Huang, E.D. Dodds, Ion Mobility Studies of Carbohydrates as Group I Adducts: Isomer Specific Collisional Cross Section Dependence on Metal Ion Radius, Anal. Chem., 85 (2013) 97289735.

[122] D.J. Harvey, C.A. Scarff, M. Edgeworth, M. Crispin, C.N. Scanlan, F. Sobott, S. Allman, K. Baruah, L. Pritchard, J.H. Scrivens, Travelling wave ion mobility and negative ion fragmentation for the structural determination of N-linked glycans, Electrophoresis, 34 (2013) 2368-2378. 
[123] S. Lee, S.J. Valentine, J.P. Reilly, D.E. Clemmer, Analyzing a mixture of disaccharides by IMS-VUVPD-MS, Int. J. Mass Spectrom., 309 (2012) 161-167.

[124] W. Hoffmann, J. Hofmann, K. Pagel, Energy-Resolved Ion Mobility-Mass Spectrometry-A Concept to Improve the Separation of Isomeric Carbohydrates, J. Am. Soc. Mass Spectrom., (2014) 19.

[125] Y. Liu, D.E. Clemmer, Characterizing Oligosaccharides Using Injected-Ion Mobility/Mass Spectrometry, Anal. Chem., 69 (1997) 2504-2509.

[126] D.S. Lee, C. Wu, H.H. Hill, Detection of carbohydrates by electrospray ionization ion mobility spectrometry following microbore high-performance liquid chromatography, Journal of Chromatography A, 822 (1998) 1-9.

[127] H. Li, K. Giles, B. Bendiak, K. Kaplan, W.F. Siems, H.H. Hill, Resolving Structural Isomers of Monosaccharide Methyl Glycosides Using Drift Tube and Traveling Wave Ion Mobility Mass Spectrometry, Anal. Chem., 84 (2012) 3231-3239.

[128] M. Zhu, B. Bendiak, B. Clowers, H. Hill, Jr., Ion mobility-mass spectrometry analysis of isomeric carbohydrate precursor ions, Anal. Bioanal. Chem., 394 (2009) 1853-1867.

[129] H. Li, B. Bendiak, K. Kaplan, E. Davis, W.F. Siems, H.H. Hill Jr, Evaluation of ion mobilitymass spectrometry for determining the isomeric heterogeneity of oligosaccharide-alditols derived from bovine submaxillary mucin, Int. J. Mass Spectrom., 352 (2013) 9-18.

[130] H.L. Li, B. Bendiak, W.F. Siems, D.R. Gang, H.H. Hill, Determining the Isomeric Heterogeneity of Neutral Oligosaccharide-Alditols of Bovine Submaxillary Mucin Using Negative Ion Traveling Wave Ion Mobility Mass Spectrometry, Anal. Chem., 87 (2015) 2228-2235.

[131] O. Hernandez, S. Isenberg, V. Steinmetz, G.L. Glish, P. Maitre, Probing Mobility-Selected Saccharide Isomers: Selective Ion-Molecule Reactions and Wavelength-Specific IR Activation, J. Phys. Chem. A, 119 (2015) 6057-6064.

[132] F. Zhu, S. Lee, S. Valentine, J. Reilly, D. Clemmer, Mannose7 Glycan Isomer Characterization by IMS-MS/MS Analysis, J. Am. Soc. Mass Spectrom., 23 (2012) 2158-2166.

[133] T. Yamagaki, A. Sato, Isomeric Oligosaccharides Analyses Using Negative-ion Electrospray Ionization Ion Mobility Spectrometry Combined with Collision-induced Dissociation MS/MS, Anal. Sci., 25 (2009) 985-988.

[134] S.Y. Vakhrushev, J. Langridge, I. Campuzano, C. Hughes, J. Peter-Katlinic, Ion mobility mass spectrometry analysis of human glycourinome, Anal. Chem., 80 (2008) 2506-2513.

[135] H.L. Li, B. Bendiak, W.F. Siems, D.R. Gang, H.H. Hill, Ion mobility mass spectrometry analysis of isomeric disaccharide precursor, product and cluster ions, Rapid Commun. Mass Spectrom., 27 (2013) 2699-2709.

[136] H. Li, B. Bendiak, W.F. Siems, D.R. Gang, H.H. Hill, Jr., Carbohydrate structure characterization by tandem ion mobility mass spectrometry (IMMS)2, Anal. Chem., 85 (2013) 27602769.

[137] M.E. Pettit, B. Harper, M.R. Brantley, T. Solouki, Collision-energy resolved ion mobility characterization of isomeric mixtures, Analyst, 14 (2015) 6886-6896.

[138] B. Clowers, P. Dwivedi, W. Steiner, H. Hill, B. Bendiak, Separation of sodiated isobaric disaccharides and trisaccharides using electrospray ionization-atmospheric pressure ion mobility-time of flight mass spectrometry, J. Am. Soc. Mass Spectrom., 16 (2005) 660-669.

[139] L.S. Fenn, J.A. McLean, Simultaneous glycoproteomics on the basis of structure using ion mobility-mass spectrometry, Mol. Biosyst., 5 (2009) 1298-1302.

[140] K. Pagel, D.J. Harvey, Ion mobility-mass spectrometry of complex carbohydrates: collision cross sections of sodiated N-linked glycans, Anal. Chem., 85 (2013) 5138-5145.

[141] Y.T. Huang, E.D. Dodds, Discrimination of Isomeric Carbohydrates as the Electron Transfer Products of Group II Cation Adducts by Ion Mobility Spectrometry and Tandem Mass Spectrometry, Anal. Chem., 87 (2015) 5664-5668.

[142] A. Guerrero, C.B. Lebrilla, New strategies for resolving oligosaccharide isomers by exploiting mechanistic and thermochemical aspects of fragment ion formation, Int. J. Mass Spectrom., 354-355 (2013) 19-25.

[143] L. Han, C. Costello, Electron Transfer Dissociation of Milk Oligosaccharides, J. Am. Soc. Mass Spectrom., 22 (2011) 997-1013. 
[144] M.M. Gaye, R. Kurulugama, D.E. Clemmer, Investigating carbohydrate isomers by IMS-CIDIMS-MS: precursor and fragment ion cross-sections, Analyst, 140 (2015) 6922-6932.

[145] N.M. Lareau, J.C. May, J.A. McLean, Non-derivatized glycan analysis by reverse phase liquid chromatography and ion mobility-mass spectrometry, The Analyst, 140 (2015) 3335-3338.

[146] D.J. Harvey, F. Sobott, M. Crispin, A. Wrobel, C. Bonomelli, S. Vasiljevic, C.N. Scanlan, C.A. Scarff, K. Thalassinos, J.H. Scrivens, Ion Mobility Mass Spectrometry for Extracting Spectra of NGlycans Directly from Incubation Mixtures Following Glycan Release: Application to Glycans from Engineered Glycoforms of Intact, Folded HIV gp120, J. Am. Soc. Mass Spectrom., 22 (2011) 568581.

[147] M. Plasencia, D. Isailovic, S. Merenbloom, Y. Mechref, D. Clemmer, Resolving and assigning N-linked glycan structural isomers from ovalbumin by IMS-MS, J. Am. Soc. Mass Spectrom., 19 (2008) 1706-1715.

[148] W.B. Struwe, J.L. Benesch, D.J. Harvey, K. Pagel, Collision cross sections of high-mannose Nglycans in commonly observed adduct states - identification of gas-phase conformers unique to $\mathrm{M}$ - $\mathrm{H}$ (-) ions, The Analyst, 140 (2015) 6799-6803.

[149] I. Capila, R.J. Linhardt, Heparin-Protein Interactions, Angewandte Chemie International Edition, 41 (2002) 390-412.

[150] Merton Bernfield, Martin Götte, Pyong Woo Park, Ofer Reizes, Marilyn L. Fitzgerald, a. John Lincecum, M. Zako, Functions of Cell Surface Heparan Sulfate Proteoglycans, Annu. Rev. Biochem., 68 (1999) 729-777.

[151] U. Lindahl, L. Thunberg, G. Bäckström, J. Riesenfeld, The antithrombin-binding sequence of heparin, Biochemical Society Transactions, 9 (1981) 499-501.

[152] Z. Hu, C. Wang, Y. Xiao, N. Sheng, Y. Chen, Y. Xu, L. Zhang, W. Mo, N. Jing, G. Hu, NDST1-dependent heparan sulfate regulates BMP signaling and internalization in lung development, Journal of Cell Science, 122 (2009) 1145-1154.

[153] H. Zhou, S. Roy, E. Cochran, R. Zouaoui, C.L. Chu, J. Duffner, G. Zhao, S. Smith, Z. Galcheva-Gargova, J. Karlgren, N. Dussault, R.Y.Q. Kwan, E. Moy, M. Barnes, A. Long, C. Honan, Y.W. Qi, Z. Shriver, T. Ganguly, B. Schultes, G. Venkataraman, T.K. Kishimoto, M402, a Novel Heparan Sulfate Mimetic, Targets Multiple Pathways Implicated in Tumor Progression and Metastasis, PLoS One, 6 (2011) e21106.

[154] N.S. Gandhi, R.L. Mancera, The Structure of Glycosaminoglycans and their Interactions with Proteins, Chem. Biol. Drug Des., 72 (2008) 455-482.

[155] H. Lortat-Jacob, A. Grosdidier, A. Imberty, Structural diversity of heparan sulfate binding domains in chemokines, Proceedings of the National Academy of Sciences, 99 (2002) 1229-1234.

[156] P. Gupta, J. McCarthy, C. Verfaillie, Stromal fibroblast heparan sulfate is required for cytokinemediated ex vivo maintenance of human long-term culture-initiating cells, Blood, 87 (1996) 32293236.

[157] L. Jin, J.P. Abrahams, R. Skinner, M. Petitou, R.N. Pike, R.W. Carrell, The anticoagulant activation of antithrombin by heparin, Proceedings of the National Academy of Sciences, 94 (1997) 14683-14688.

[158] A.K. Powell, E.A. Yates, D.G. Fernig, J.E. Turnbull, Interactions of heparin/heparan sulfate with proteins: Appraisal of structural factors and experimental approaches, Glycobiology, 14 (2004) 17R-30R.

[159] D.L. Rabenstein, Heparin and heparan sulfate: structure and function, Natural Product Reports, 19 (2002) 312-331.

[160] J.R. Bishop, M. Schuksz, J.D. Esko, Heparan sulphate proteoglycans fine-tune mammalian physiology, Nature, 446 (2007) 1030-1037.

[161] M. Ly, F.E. Leach, T.N. Laremore, T. Toida, I.J. Amster, R.J. Linhardt, The proteoglycan bikunin has a defined sequence, Nat Chem Biol, 7 (2011) 827-833.

[162] S. Faham, R.E. Hileman, J.R. Fromm, R.J. Linhardt, D.C. Rees, Heparin Structure and Interactions with Basic Fibroblast Growth Factor, Science, 271 (1996) 1116-1120.

[163] E. Sisu, S. Tripathy, J.-M. Mallet, P.-A. Driguez, J.-P. Hérault, P. Sizun, J.-M. Herbert, M. Petitou, P. Sinay, Synthesis of new conformationally constrained pentasaccharides as molecular probes to investigate the biological activity of heparin, Biochimie, 85 (2003) 91-99. 
[164] Y. Seo, M.R. Schenauer, J.A. Leary, Biologically relevant metal-cation binding induces conformational changes in heparin oligosaccharides as measured by ion mobility mass spectrometry, Int. J. Mass Spectrom., 303 (2011) 191-198.

[165] S. Ricard-Blum, O. Féraud, H. Lortat-Jacob, A. Rencurosi, N. Fukai, F. Dkhissi, D. Vittet, A. Imberty, B.R. Olsen, M. van der Rest, Characterization of Endostatin Binding to Heparin and Heparan Sulfate by Surface Plasmon Resonance and Molecular Modeling: Role of Divalent Cations, J. Biol. Chem., 279 (2004) 2927-2936.

[166] B. Lages, S.S. Stivala, Interaction of the polyelectrolyte heparin with copper(II) and calcium, Biopolymers, 12 (1973) 127-143.

[167] F. Chevalier, J. Angulo, R. Lucas, Pedro M. Nieto, M. Martín-Lomas, The Heparin-Ca2+ Interaction: Structure of the Ca2+ Binding Site, Eur. J. Org. Chem., 2002 (2002) 2367-2376.

[168] R. González-Iglesias, M.a.A. Pajares, C. Ocal, J. Carlos Espinosa, B. Oesch, M.a. Gasset, Prion Protein Interaction with Glycosaminoglycan Occurs with the Formation of Oligomeric Complexes Stabilized by Cu(II) Bridges, J. Mol. Biol., 319 (2002) 527-540.

[169] R.F. Parrish, W.R. Fair, Selective binding of zinc ions to heparin rather than to other glycosaminoglycans, Biochem. J., 193 (1981) 407-410.

[170] S.R. Srinivasan, B. Radhakrishnamurthy, G.S. Berenson, Studies on the interaction of heparin with serum lipoproteins in the presence of $\mathrm{Ca} 2+, \mathrm{Mg} 2+$, and $\mathrm{Mn} 2+$, Archives of Biochemistry and Biophysics, 170 (1975) 334-340.

[171] F. Chevalier, R. Lucas, J. Angulo, M. Martin-Lomas, P.M. Nieto, The heparin-Ca2+ interaction: the influence of the O-sulfation pattern on binding, Carbohydr. Res., 339 (2004) 975-983.

[172] M.R. Schenauer, J.K. Meissen, Y. Seo, J.B. Ames, J.A. Leary, Heparan Sulfate Separation, Sequencing, and Isomeric Differentiation: Ion Mobility Spectrometry Reveals Specific Iduronic and Glucuronic Acid-Containing Hexasaccharides, Anal. Chem., 81 (2009) 10179-10185.

[173] R. Miller, W. Wei, R. Schwörer, O. Zubkova, P. Tyler, J. Turnbull, J. Leary, Composition, sequencing and ion mobility mass spectrometry of heparan sulfate-like octasaccharide isomers differing in glucuronic and iduronic acid content, Eur. J. Mass Spectrom., 21 (2015) 245-254.

[174] E. Gray, B. Mulloy, T.W. Barrowcliffe, Heparin and low-molecular-weight heparin, Thromb. Haemost., 99 (2008) 807-818.

[175] D.R. Ferro, A. Provasoli, M. Ragazzi, B. Casu, G. Torri, V. Bossennec, B. Perly, P. Sinaÿ, M. Petitou, J. Choay, Conformer populations of 1-iduronic acid residues in glycosaminoglycan sequences, Carbohydr. Res., 195 (1990) 157-167.

[176] P.N. Sanderson, T.N. Huckerby, I.A. Nieduszynski, Conformational equilibria of $\alpha$-L-iduronate residues in disaccharides derived from heparin, Biochem. J., 243 (1987) 175-181.

[177] Y. Seo, A. Andaya, J.A. Leary, Preparation, Separation, and Conformational Analysis of Differentially Sulfated Heparin Octasaccharide Isomers Using Ion Mobility Mass Spectrometry, Anal. Chem., 84 (2012) 2416-2423.

[178] B. Mulloy, M.J. Forster, Conformation and dynamics of heparin and heparan sulfate, Glycobiology, 10 (2000) 1147-1156.

[179] M. Hermannová, A.-M. Iordache, K. Slováková, V. Havlíček, H. Pelantová, K. Lemr, Arrival time distributions of product ions reveal isomeric ratio of deprotonated molecules in ion mobilitymass spectrometry of hyaluronan-derived oligosaccharides, Journal of Mass Spectrometry, 50 (2015) 854-863.

[180] M. Kailemia, M. Park, D. Kaplan, A. Venot, G.-J. Boons, L. Li, R. Linhardt, I.J. Amster, HighField Asymmetric-Waveform Ion Mobility Spectrometry and Electron Detachment Dissociation of Isobaric Mixtures of Glycosaminoglycans, J. Am. Soc. Mass Spectrom., 25 (2014) 258-268.

[181] J.J. Wolff, T.N. Laremore, F.E. Leach, R.J. Linhardt, I.J. Amster, Electron capture dissociation, electron detachment dissociation, and infrared multiphoton dissociation of sucrose octasulfate, European journal of mass spectrometry (Chichester, England), 15 (2009) 275-281.

[182] M.S. Motawia, I. Damager, C.E. Olsen, B.L. Møller, S.B. Engelsen, S. Hansen, L.H. Øgendal, R. Bauer, Comparative Study of Small Linear and Branched $\alpha$-Glucans Using Size Exclusion Chromatography and Static and Dynamic Light Scattering\#, Biomacromolecules, 6 (2005) 143-151.

[183] V.G. Murphy, B. Zaslow, A.D. French, The structure of V amylose dehydrate: A combined Xray and stereochemical approach, Biopolymers, 14 (1975) 1487-1501. 
[184] K. Nishinari, K. Kohyama, P.A. Williams, G.O. Phillips, W. Burchard, K. Ogino, Solution properties of pullulan, Macromolecules, 24 (1991) 5590-5593.

[185] J.-H. Huang, E.J. Bakx, H. Gruppen, H.A. Schols, Characterisation of 3-aminoquinolinederivatised isomeric oligogalacturonic acid by travelling-wave ion mobility mass spectrometry, Rapid Commun. Mass Spectrom., 27 (2013) 2279-2285.

[186] K. Kaneshiro, Y. Fukuyama, S. Iwamoto, S. Sekiya, K. Tanaka, Highly Sensitive MALDI Analyses of Glycans by a New Aminoquinoline-Labeling Method Using 3-Aminoquinoline/ $\alpha$-Cyano4-hydroxycinnamic Acid Liquid Matrix, Anal. Chem., 83 (2011) 3663-3667.

[187] A.G.M. Leijdekkers, J.-H. Huang, E.J. Bakx, H. Gruppen, H.A. Schols, Identification of novel isomeric pectic oligosaccharides using hydrophilic interaction chromatography coupled to travelingwave ion mobility mass spectrometry, Carbohydr. Res., 404 (2015) 1-8.

[188] B. Plancot, G. Vanier, F. Maire, M. Bardor, P. Lerouge, J.M. Farrant, J. Moore, A. Driouich, M. Vicré-Gibouin, C. Afonso, C. Loutelier-Bourhis, Structural characterization of arabinoxylans from two African plant species Eragrostis nindensis and Eragrostis tef using various mass spectrometric methods, Rapid Commun. Mass Spectrom., 28 (2014) 908-916.

[189] M.R. Schenauer, J.A. Leary, An ion mobility-mass spectrometry investigation of monocyte chemoattractant protein-1, Int. J. Mass Spectrom., 287 (2009) 70-76.

[190] Y. Seo, A. Andaya, C. Bleiholder, J.A. Leary, Differentiation of CC vs CXC Chemokine Dimers with GAG Octasaccharide Binding Partners: An Ion Mobility Mass Spectrometry Approach, J. Am. Chem. Soc., 135 (2013) 4325-4332.

[191] Y. Tian, L. Han, A.C. Buckner, B.T. Ruotolo, Collision Induced Unfolding of Intact Antibodies: Rapid Characterization of Disulfide Bonding Patterns, Glycosylation, and Structures, Anal. Chem., 87 (2015) 11509-11515.

[192] B.J. McCullough, J.M. Kalapothakis, W. Chin, K. Taylor, D.J. Clarke, H. Eastwood, D. Campopiano, D. MacMillan, J. Dorin, P.E. Barran, Binding a heparin derived disaccharide to defensin inspired peptides: insights to antimicrobial inhibition from gas-phase measurements, Phys. Chem. Chem. Phys., 12 (2010) 3589-3596.

[193] S.R. Harvey, C.E. MacPhee, B.F. Volkman, P.E. Barran, The association and aggregation of the metamorphic chemokine lymphotactin with fondaparinux: from $\mathrm{nm}$ molecular complexes to [small mu ]m molecular assemblies, Chem. Commun., (2016).

[194] H. Li, B. Bendiak, W. Siems, D. Gang, H. Hill, Jr., Ion mobility-mass correlation trend line separation of glycoprotein digests without deglycosylation, Int. J. Ion Mobil. Spec., 16 (2013) 105115.

[195] A.J. Creese, H.J. Cooper, Separation and Identification of Isomeric Glycopeptides by High Field Asymmetric Waveform Ion Mobility Spectrometry, Anal. Chem., 84 (2012) 2597-2601.

[196] G.N. Ulasi, A.J. Creese, S.X. Hui, C.W. Penn, H.J. Cooper, Comprehensive mapping of Oglycosylation in flagellin from Campylobacter jejuni 11168: A multienzyme differential ion mobility mass spectrometry approach, Proteomics, 15 (2015) 2733-2745.

[197] K. Brandenburg, O. Holst, Glycolipids: Distribution and Biological Function, in: eLS, John Wiley \& Sons, Ltd, 2001.

[198] J.B. Robbins, R. Schneerson, Evaluating the Haemophilus influenzae Type b Conjugate Vaccine PRP-D, New England Journal of Medicine, 323 (1990) 1415-1416.

[199] B.D. Gessner, R.A. Adegbola, The impact of vaccines on pneumonia: Key lessons from Haemophilus influenzae type b conjugate vaccines, Vaccine, 26, Supplement 2 (2008) B3-B8.

[200] E. Miller, D. Salisbury, M. Ramsay, Planning, registration, and implementation of an immunisation campaign against meningococcal serogroup C disease in the UK: a success story, Vaccine, 20, Supplement 1 (2001) S58-S67.

[201] J.T. Poolman, Pneumococcal vaccine development, Expert Review of Vaccines, 3 (2004) 597604.

[202] S.P. Lockhart, J.G. Hackell, B. Fritzell, Pneumococcal conjugate vaccines: emerging clinical information and its implications, Expert Review of Vaccines, 5 (2006) 553-564.

[203] A.H. Merrill Jr, M.D. Wang, M. Park, M.C. Sullards, (Glyco)sphingolipidology: an amazing challenge and opportunity for systems biology, Trends in Biochemical Sciences, 32 (2007) 457-468. 
[204] A.H. Merrill, T.H. Stokes, A. Momin, H. Park, B.J. Portz, S. Kelly, E. Wang, M.C. Sullards, M.D. Wang, Sphingolipidomics: a valuable tool for understanding the roles of sphingolipids in biology and disease, Journal of Lipid Research, 50 (2009) S97-S102.

[205] S. Sonnino, V. Chigorno, Ganglioside molecular species containing C18- and C20-sphingosine in mammalian nervous tissues and neuronal cell cultures, Biochimica et Biophysica Acta (BBA) Reviews on Biomembranes, 1469 (2000) 63-77.

[206] R. Yu, Y.-T. Tsai, T. Ariga, Functional Roles of Gangliosides in Neurodevelopment: An Overview of Recent Advances, Neurochem Res, 37 (2012) 1230-1244.

[207] E. Posse de Chaves, S. Sipione, Sphingolipids and gangliosides of the nervous system in membrane function and dysfunction, FEBS Letters, 584 1748-1759.

[208] G. van Echten-Deckert, J. Walter, Sphingolipids: Critical players in Alzheimer's disease, Progress in Lipid Research, 51 (2012) 378-393.

[209] T. Ariga, M.P. McDonald, R.K. Yu, Thematic Review Series: Sphingolipids. Role of ganglioside metabolism in the pathogenesis of Alzheimer's disease-a review, Journal of Lipid Research, 49 (2008) 1157-1175.

[210] T. Kolter, K. Sandhoff, Principles of Lysosomal Membrane Digestion: Stimulation of Sphingolipid Degradation by Sphingolipid Activator Proteins and Anionic Lysosomal Lipids, Annual Review of Cell and Developmental Biology, 21 (2005) 81-103.

[211] X. Zhang, F.L. Kiechle, Glycosphingolipids in Health and Disease, Annals of Clinical \& Laboratory Science, 34 (2004) 3-13.

[212] M.R. Wenk, The emerging field of lipidomics, Nat Rev Drug Discov, 4 (2005) 594-610.

[213] J. Li, R.W. Purves, J.C. Richards, Coupling Capillary Electrophoresis and High-Field Asymmetric Waveform Ion Mobility Spectrometry Mass Spectrometry for the Analysis of Complex Lipopolysaccharides, Anal. Chem., 76 (2004) 4676-4683.

[214] S.N. Jackson, M. Ugarov, T. Egan, J.D. Post, D. Langlais, J. Albert Schultz, A.S. Woods, MALDI-ion mobility-TOFMS imaging of lipids in rat brain tissue, Journal of Mass Spectrometry, 42 (2007) 1093-1098.

[215] S.N. Jackson, B. Colsch, T. Egan, E.K. Lewis, J.A. Schultz, A.S. Woods, Gangliosides' analysis by MALDI-ion mobility MS, Analyst, 136 (2011) 463-466.

[216] S.N. Jackson, D. Barbacci, T. Egan, E.K. Lewis, J.A. Schultz, A.S. Woods, MALDI-ion mobility mass spectrometry of lipids in negative ion mode, Analytical Methods, 6 (2014) 5001-5007.

[217] S. Jackson, M. Ugarov, J. Post, T. Egan, D. Langlais, J.A. Schultz, A. Woods, A study of phospholipids by ion mobility TOFMS, J. Am. Soc. Mass Spectrom., 19 (2008) 1655-1662.

[218] Y. Mechref, Y. Hu, A. Garcia, A. Hussein, Identifying cancer biomarkers by mass spectrometry-based glycomics, Electrophoresis, 33 (2012) 1755-1767.

[219] H. Liu, N. Zhang, D. Wan, M. Cui, Z. Liu, S. Liu, Mass spectrometry-based analysis of glycoproteins and its clinical applications in cancer biomarker discovery, Clinical Proteomics, 11 (2014) 1-9.

[220] D. Isailovic, R.T. Kurulugama, M.D. Plasencia, S.T. Stokes, Z. Kyselova, R. Goldman, Y. Mechref, M.V. Novotny, D.E. Clemmer, Profiling of Human Serum Glycans Associated with Liver Cancer and Cirrhosis by IMS-MS, Journal of Proteome Research, 7 (2008) 1109-1117.

[221] A.A. Shvartsburg, W.F. Danielson, R.D. Smith, High-Resolution Differential Ion Mobility Separations Using Helium-Rich Gases, Anal. Chem., 82 (2010) 2456-2462.

[222] S. Lee, M.A. Ewing, F.M. Nachtigall, R.T. Kurulugama, S.J. Valentine, D.E. Clemmer, Determination of Cross Sections by Overtone Mobility Spectrometry: Evidence for Loss of Unstable Structures at Higher Overtones, J. Phys. Chem. B, 114 (2010) 12406-12415.

[223] S.J. Valentine, R.T. Kurulugama, D.E. Clemmer, Overtone Mobility Spectrometry: Part 3. On the Origin of Peaks, J. Am. Soc. Mass Spectrom., 22 (2011) 804-816.

[224] R.T. Kurulugama, F.M. Nachtigall, S.J. Valentine, D.E. Clemmer, Overtone Mobility Spectrometry: Part 4. OMS-OMS Analyses of Complex Mixtures, J. Am. Soc. Mass Spectrom., 22 (2011) 2049-2060.

[225] M.A. Ewing, S.M. Zucker, S.J. Valentine, D.E. Clemmer, Overtone Mobility Spectrometry: Part 5. Simulations and Analytical Expressions Describing Overtone Limits, J. Am. Soc. Mass Spectrom., 24 (2013) 615-621. 
[226] M.A. Ewing, C.R.P. Conant, S.M. Zucker, K.J. Griffith, D.E. Clemmer, Selected Overtone Mobility Spectrometry, Anal. Chem., 87 (2015) 5132-5138.

[227] S. Valentine, S. Stokes, R. Kurulugama, F. Nachtigall, D. Clemmer, Overtone mobility spectrometry: Part 2. Theoretical considerations of resolving power, J. Am. Soc. Mass Spectrom., 20 (2009) 738-750.

[228] K. Giles, J. Wildgoose, S. Pringle, J. Garside, P. Carney, P. Nixon, D. Langridge, Design and utility of a multi-pass cyclic ion mobility separator, in: 62nd ASMS Conference, Baltimore, 2014.

[229] S.I. Merenbloom, R.S. Glaskin, Z.B. Henson, D.E. Clemmer, High-Resolution Ion Cyclotron Mobility Spectrometry, Anal. Chem., 81 (2009) 1482-1487.

[230] R.S. Glaskin, M.A. Ewing, D.E. Clemmer, Ion Trapping for Ion Mobility Spectrometry Measurements in a Cyclical Drift Tube, Anal. Chem., 85 (2013) 7003-7008.

[231] L. Cipolla, F. Peri, C. Airoldi, Glycoconjugates in cancer therapy, Anti-Cancer Agents Med. Chem., 8 (2008) 92-121.

[232] C. Jones, Vaccines based on the cell surface carbohydrates of pathogenic bacteria, Anais da Academia Brasileira de Ciências, 77 (2005) 293-324.

[233] J.R. Mascola, G. Stiegler, T.C. VanCott, H. Katinger, C.B. Carpenter, C.E. Hanson, H. Beary, D. Hayes, S.S. Frankel, D.L. Birx, M.G. Lewis, Protection of macaques against vaginal transmission of a pathogenic HIV-1/SIV chimeric virus by passive infusion of neutralizing antibodies, Nat Med, 6 (2000) 207-210.

[234] R.D. Astronomo, D.R. Burton, Carbohydrate vaccines: developing sweet solutions to sticky situations?, Nat Rev Drug Discov, 9 (2010) 308-324.

[235] D.J. Harvey, M. Crispin, C. Bonomelli, J.H. Scrivens, Ion Mobility Mass Spectrometry for Ion Recovery and Clean-Up of MS and MS/MS Spectra Obtained from Low Abundance Viral Samples, J. Am. Soc. Mass Spectrom., 26 (2015) 1754-1767. 